\title{
Switched-RC Beamforming Receivers in Advanced CMOS \\ Theory and Design
}

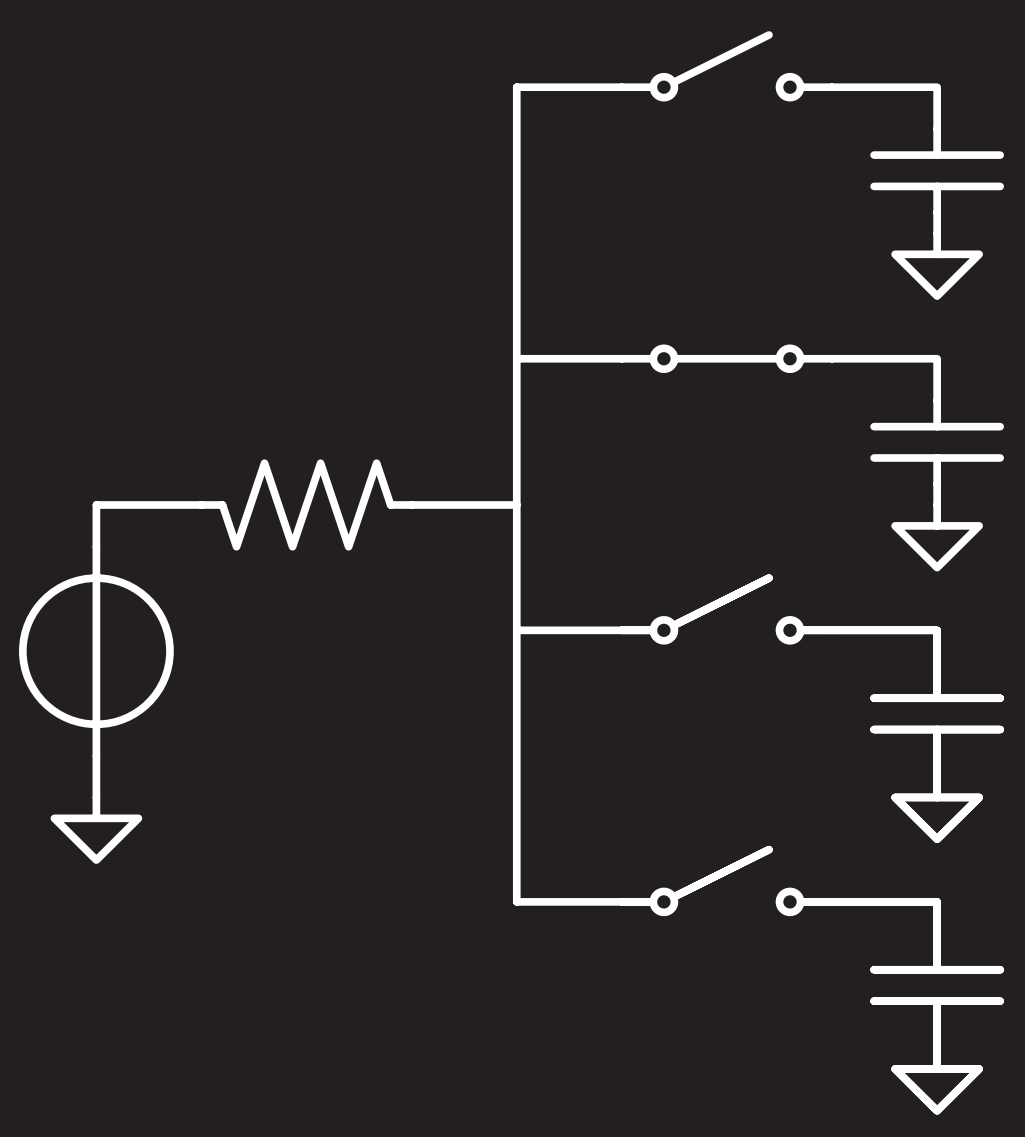

Michiel Soer 


\title{
Switched-RC BEAmForming \\ Receivers in Advanced CMOS
}

\author{
TheOrY AND DEsIgN
}

Michiel Soer 
Samenstelling promotiecommissie:

Voorzitter en secretaris:

Prof.dr.ir. A.J. Mouthaan

Universiteit Twente

Promotor:

Prof.dr.ir. F.E. van Vliet

Universiteit Twente

Assistent-promotor:

Dr.ing. E.A.M. Klumperink

Universiteit Twente

Leden:

Prof.dr.ir. B. Nauta

Universiteit Twente

Prof.ir. A.J.M. van Tuijl

Universiteit Twente

Prof.dr.ir. D. Schreurs

Katholieke Universiteit Leuven

Prof.dr. J.R. Long

Technische Universiteit Delft

Referent:

Dr. A. Cathelin

STMicroelectronics

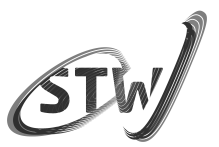

Enabling new technology
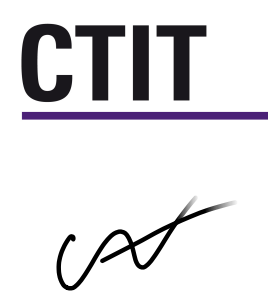

This research was financially supported by the Dutch Technology Foundation STW (07620).

Centre for Telematics and Information Technology

P.O. Box 217, 7500 AE

Enschede, The Netherlands

Centre for Array Technology.

ISSN: $\quad$ 1381-3617 (CTIT Ph.D. Thesis Series No. 12-233)

ISBN: 978-90-365-3436-9

DOI: $\quad$ http://dx.doi.org/10.3990/1.9789036534369

Copyright (C) 2012 by Michiel Soer, Enschede, The Netherlands All rights reserved.

Typeset with $\mathrm{LAT}_{\mathrm{E}} \mathrm{X}$.

This thesis was printed by Gildeprint Drukkerijen, The Netherlands. 


\title{
Switched-RC BeAmForming ReCEIVERs in Advanced CMOS
}

\author{
TheORY AND DESIGN
}

\section{PROEFSCHRIFT}

\author{
ter verkrijging van
}

de graad van doctor aan de Universiteit Twente, op gezag van de rector magnificus, prof. dr. H. Brinksma,

volgens besluit van het College voor Promoties in het openbaar te verdedigen op donderdag 22 november 2012 om 16:45 uur

door

Michiel Cornelius Marinus Soer geboren op 19 juli 1984

te Schoonhoven 
Dit proefschrift is goedgekeurd door:

de promotor prof.dr.ir. F.E van Vliet

de assistent-promotor dr.ing. E.A.M. Klumperink 


\section{Samenvatting}

In consumentenelektronica wordt de daadwerkelijke draadloze verbinding opgebouwd door analoge radio zenders en ontvangers. Deze radio's zijn voornamelijk omnidirectioneel, zodanig dat zenders hun energy in alle richtingen verspreiden en zenders gevoelig zijn voor signalen die uit elke richting kunnen komen. Vanwege deze eigenschappen wordt met de toenemende hoeveelheid aan draadloze verbindingen de interferentie tussen apparaten en diensten steeds problematischer. Voor het sturen van en luisteren naar radio signalen uit specifieke richtingen is het noodzakelijk om meerdere antennes aan de radio's te verbinden en precieze tijdvertragingen tussen deze antennes aan te brengen. De resulterende "bundelvorming"kan begrepen worden als een soort van ruimtelijk filter.

De implementatie van een dergelijk systeem in consumentenelektronica vereist dat circuits met tijdsvertraging en/of fasedraaing worden toegevoegd aan de bestaande radio architecturen. Deze circuits zijn lastig te fabriceren in de geïntegreerde circuit (IC) technologie die gebruikt wordt voor consumentenapparaten. Daarom onderzoekt dit proefschrift de uitvoering van bundelvormende ontvangers in geavanceerd complementary metal oxide semiconductor (CMOS) IC technologie.

Het bundelvormingsmechanisme is gedefinieerd in de vorm van antenne vertragingen in het radiofrequentie (RF) domein. Analyse van de bundelvormingseigenschappen laat zien dat voor smalbandige systemen de fasedraaing verplaats kan worden tot na de frequentietranslatie, naar het tussenfrequentie (IF) domein. De translatie kan meteen de 90-graden-uit-fase signalen genereren die nodig zijn voor een fasedraaier van het vector-modulator type. Bovendien vermindert de vertaling naar het IF domein de benodigde bandbreedte in de fasedraaier.

Een nadeel van IF bundelvorming bestaat eruit dat de mixers en versterkers in het eerste stuk van de ontvanger bloot staan aan de ongefilterde verstoorders, zodat de vervorming die gegenereerd wordt door deze componenten laag gehouden moet worden. Het is bekend dat een lage vervorming in CMOS technologie bereikt kan worden door passieve schakelende circuits, bijvoorbeeld door geschakelde-weerstandcapaciteit mixers en spanningssamplers. Met een uitvoerige analyse wordt aangetoond dat het basis bouwblok van deze circuits bestaat uit een enkel geschakelde-weerstand- 
capaciteit stroomlus. Bovendien wordt er geconcludeerd dat de overdrachtsfunctie en ruisbijdrage van deze lus afhangen van de verhouding tussen de $\mathrm{RC}$ tijdconstante en de tijd dat de schakelaar aan staat. Twee duidelijke werkingsgebieden worden aangeduid, waarvan de eerste lage ruis eigenschappen heeft voor mixer toepassingen en de tweede hoge bandbreedtes aanbiedt voor sampler toepassingen.

De goede vervormingseigenschappen van deze circuits worden optimaal benut, indien de mixer-eerst architectuur wordt gebruikt, waarin de frequentietranslatie met een geschakelde-RC mixer wordt uitgevoerd voordat er versterking plaatsvind. Doordat de versterking plaatsvindt in het IF domein, is het mogelijk om feedback toe te passen teneinde de vervorming te verlagen. Deze technieken zijn toegepast in een 65-nm CMOS ontvanger IC, met een derde-orde interceptie punt (IIP3) van $11 \mathrm{dBm}$ en een ruisgetal (NF) van 6.5 dB. De RF frequentieband is kiesbaar tussen $200 \mathrm{MHz}$ en $2.0 \mathrm{GHz}$, hetgeen mogelijk wordt gemaakt door de van nature breedbandige mixer.

Vervolgens is het ontwerp van de vector-modulator fasedraaier aan de beurt, waarvoor het nodig is om sinus en cosinus weging toe te passen op de 90-graden-uit-fase signalen uit de mixer. Een goede benadering voor deze weging kan worden verkregen door een circuit met ladingsherverdeling en geschakelde capaciteiten toe te passen. Omdat de resulterende fasedraaing afhankelijk is van de verhoudingen tussen capaciteiten, is deze uiterst nauwkeurig gedefinieerd in een CMOS proces. De implementatie van een 4-element 1.0-tot-4.0 GHz geïntegreerde ontvanger in 65-nm CMOS laat zien dat de 5-bit fasedraaiers een root-mean-square (RMS) fasefout halen van slechts 1.4 graden, met een RMS versterkingsfout van $0.4 \mathrm{~dB}$. Bovendien wordt de extra 3-bit amplitudecontrole van de vector modulators gebruikt in een demonstratie van het onderdrukken van verstoorders met meer dan $20 \mathrm{~dB}$ door middel van een bundelvormingsalgoritme. Echter, het blijkt uit simulaties dat de vervorming (-1 dBm IIP3 binnen de band) gelimiteerd is door de actieve trappen in de bundelvormer.

Vandaar dat de overgang naar een compleet passieve bundelvormer gewenst is. Hiervoor zullen echter de koppelingen tussen de geschakelde-RC circuits in de mixer en de fasedraaier gemodelleerd moeten worden. Deze koppelingen kunnen gezien worden als een effectieve weerstandsbelasting en er wordt een techniek gepresenteerd waarmee de lading benodigd voor de aanpassing van de ingangsimpedantie hergebruikt kan worden voor de fasedraaier. Met deze technieken is een volledig passieve 4-element 1.5-tot-5.0 GHz bundelvormende ontvanger geïmplementeerd in 65-nm CMOS, met een IIP3 van $13 \mathrm{dBm}$ binnen de frequentieband en een NF van $18 \mathrm{~dB}$. Bovendien werkt de mixer als een mee schuivend frequentiefilter aan de ingang, waardoor het compressiepunt wordt verhoogd van $2 \mathrm{dBm}$ binnen de band tot een zeer hoge $12 \mathrm{dBm}$ buiten de band. De resultaten tonen aan dat deze topologie voor een bundelvormer in CMOS zeer bestendig is tegen sterke verstoorders. 


\section{Abstract}

In consumer electronic devices, analog radio transmitters and receivers provide the wireless links between devices. These radios are mostly omni-directional, i.e. transmitters send their energy in all directions and receivers listen to signals from all directions. As a result, interference between devices and services is becoming an increasing problem as more wireless connectivity is added. In order to steer radio signals toward their destination and to receive from a specific direction, it is necessary to add multiple antennas to the radios and to control their precise relative delays. The resulting beamforming can be understood to be a form of spatial filtering.

For the implementation of such a system in consumer electronics, it is required to add additional time delay and/or phase shift circuits to the existing radio architectures. These circuits are challenging to fabricate in the integrated circuit (IC) technology used for consumer devices. Therefore, this thesis investigates the implementation of beamforming receivers in advanced complementary metal oxide semiconductor (CMOS) IC technology.

The beamforming is defined in terms of the antenna delays in the radio frequency (RF) domain. An analysis of the beamforming properties reveals that in a narrowband system, the phase shift can be moved until after downconversion to the intermediate frequency (IF) domain. The downconversion can then generate 90-degree-out-of-phase signals, necessary for the operation of a vector-modulator type phase shifter. Moreover, the shift to the IF domain significantly reduces the required bandwidth of the phase shifter implementation.

A disadvantage of IF beamforming is that the frontend mixers and amplifiers are subject to the unfiltered interferers, requiring a high linearity for these components. In CMOS technology, high linearity is achieved by passive switching circuits, such as switched-RC mixers and voltage samplers. A rigorous analysis reveals that a switchedresistor-capacitor current loop is the basic building block of these systems. It is also concluded that the transfer function and noise contribution of this loop depend on the ratio between the $\mathrm{RC}$ time constant and the switch-on time. Two distinctive operating regions are identified, one with low noise properties for mixer applications, and one with high bandwidth properties for sampling applications. 
In order to take advantage of the good linearity properties of these circuits, the mixer-first architecture is proposed. In this architecture, downconversion with an inherently linear switched-RC mixing-region mixer takes place before amplification. By postponing the amplification to the IF domain, feedback can be applied to increase amplifier linearity. These concepts are demonstrated in a 65-nm CMOS receiver IC, achieving an $11 \mathrm{dBm}$ input-referred third-order intercept point (IIP3) and a $6.5 \mathrm{~dB}$ noise figure $(\mathrm{NF})$, which translates into $79 \mathrm{~dB}$ of spurious free dynamic range in 1 $\mathrm{MHz}$ bandwidth. Due to the wideband nature of the mixer, a tunable RF input band of $200 \mathrm{MHz}$ up to $2 \mathrm{GHz}$ is achieved.

Next, the design of the IF domain vector-modulator phase shifter is considered, in which it is required to apply sine and cosine weighting on the 90-degree-out-ofphase signals from the mixer. It is shown that a good approximation of the weighting can be achieved by a charge-redistribution switched-capacitor circuit. The resulting phase shift is a function of capacitor ratios, which are very well defined in CMOS processes. A 4-element 1.0-to-4.0 GHz integrated receiver is implemented in 65-nm CMOS, including 5-bit phase shifters with a root-mean-square (RMS) phase error of 1.4 degrees and a RMS gain error of $0.4 \mathrm{~dB}$. Using the additional 3-bit gain control of the vector modulator, a beamforming infererence nulling algorithm is demonstrated which is able to suppress interferers by more than $20 \mathrm{~dB}$. In this design, simulations indicate that the limiting factor in linearity $(-1 \mathrm{dBm}$ in-band IIP3) is formed by the active stages in the beamforming network.

Therefore, the transition to a fully passive, switched-RC beamforming receiver is desired. This requires the modeling of the coupling between the mixing-region mixer and the sampling-region phase shifter. By defining an effective input resistance for the two stages, its loading effects can be examined, and it is found that the charge dissipation in the phase shifter can be used to implement input matching at the mixer input. Using this design framework, a fully-passive 4-element 1.5-to-5.0 GHz beamforming receiver is implemented in 65-nm CMOS, with an in-band IIP3 of 13 $\mathrm{dBm}$ and a $\mathrm{NF}$ of $18 \mathrm{~dB}$. It is also found that intrinsic filtering at the mixer input occurs, boosting the compression point from $2 \mathrm{dBm}$ in-band to a very high $12 \mathrm{dBm}$ out-of-band, demonstrating the high resilience of this CMOS beamforming topology to strong interferers. 


\section{Contents}

Samenvatting $\quad$ i

Abstract

1 Introduction 1

1.1 Wireless Communications . . . . . . . . . . . . . . . . . 1

1.2 CMOS Integrated Circuit Technology . . . . . . . . . . . . . 2

1.3 Multi-Antenna Wireless Systems . . . . . . . . . . . . . . . . 4

1.4 Motivation and Thesis Outline . . . . . . . . . . . . . 7

2 Phased-Array Downconverting Receivers 9

2.1 Linear Array Theory . . . . . . . . . . . . . . . . . . . 9 9

2.1.1 Beam Steering . . . . . . . . . . . . . . . 10

2.1.2 Narrowband Phase Shifter Approximation . . . . . . . . . . . 13

2.1.3 Directivity ....................... 14

2.1.4 Amplitude Tapering . . . . . . . . . . . . . . . . . 17

2.1.5 Null Steering . . . . . . . . . . . . . . . . . 18

2.1.6 Random Errors . . . . . . . . . . . . . . . . . . . . 20

2.1.7 Phase Quantization Errors . . . . . . . . . . . . 22

2.1.8 Amplitude Quantization Errors . . . . . . . . . . . . . . 23

2.2 Frequency Translation and Beamforming . . . . . . . . . . . . . . . 24

2.2.1 Effects of Phase Shift and Time Delay . . . . . . . . . . . . . . 24

2.2 .2 Transformations ................... 27

2.3 RF System Design Aspects . . . . . . . . . . . . . . . . . 30

2.3.1 Bandwidth and Architecture . . . . . . . . . . . . . 31

2.3.2 Linearity and Noise . . . . . . . . . . . . . . . . . . 32

2.4 Conclusions . . . . . . . . . . . . . . . . . . . . . . 33 
3 Highly-Linear Mixer-First Receiver Front-Ends $\quad 34$

3.1 Switched-RC Mixers and Samplers . . . . . . . . . . . . . 35

$3.1 .1 \quad$ LPTV Systems . . . . . . . . . . . . . . . . . 36

3.1.2 Decomposition into Polyphase Kernels . . . . . . . . . . . . 38

3.1 .3 Kernel Analysis . . . . . . . . . . . . . . . . . . . . . . . . 40

3.1.4 Polyphase Multi-path Analysis . . . . . . . . . . . . . 44

3.1 .5 Noise . . . . . . . . . . . . . . . . . . 46

3.1.6 Application to Low-Noise Passive Mixers . . . . . . . . . 50

3.2 Implementation in $65-\mathrm{nm}$ CMOS . . . . . . . . . . . 52

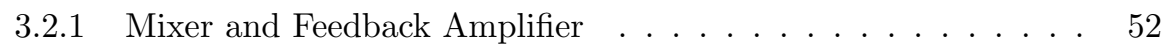

3.2 .2 Clock Generation . . . . . . . . . . . . . . . . . . 54

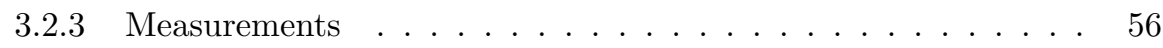

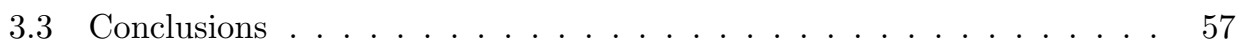

4 Approximate-Sine Vector Modulator Beamforming $\quad \mathbf{5 8}$

4.1 Switched-Capacitor Phase Shifter . . . . . . . . . . . . . . 58

4.1.1 Vector Modulator Principle . . . . . . . . . . . . . . . 59

4.1.2 Sine/Cosine Approximation for Non-Uniform Weighting . . . . 60

4.1.3 Amplitude Control for Null Steering . . . . . . . . . . . . 64

4.2 Implementation in $65-\mathrm{nm}$ CMOS . . . . . . . . . . . . 65

4.2 .1 Mixer . . . . . . . . . . . . . . . . . 66

4.2 .2 Vector Modulator . . . . . . . . . . . . . . 67

4.2 .3 Element Summing . . . . . . . . . . . . . . . . . . . . 68

4.2 .4 Clock generation . . . . . . . . . . . . . . . . . . 69

4.2 .5 Measurements . . . . . . . . . . . . . . . 70

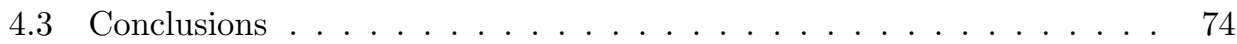

5 All-Passive Switched-Capacitor Phased Array $\quad 75$

5.1 Coupling of Switched-RC Loops . . . . . . . . . . . . . . 76

5.1 .1 Mixing Region Loops _. . . . . . . . . . . . . . . . . . . . 78

5.1.2 Input-Matched Passive Mixer . . . . . . . . . . . . . . . 79

5.1.3 Sampling Region Loops and Resistor Simulation _... . . . . 82

5.1 .4 Phase Shifting . . . . . . . . . . . . . . . . . . . . 83

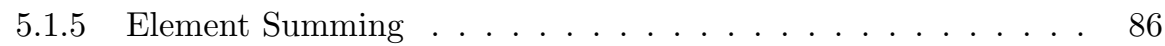

5.2 Implementation in $65-\mathrm{nm}$ CMOS $\ldots \ldots \ldots \ldots \ldots . \ldots \ldots$

$5.2 .1 \quad$ Mixer . . . . . . . . . . . . . . . . . . 88

$5.2 .2 \quad$ Phase Shifter Slice . . . . . . . . . . . . . . . . . . . . . . . 89

5.2 .3 Conversion Loss and Noise Figure . . . . . . . . . . . . . . 90

5.2 .4 Clock Generation . . . . . . . . . . . . . . . . . . . . 91

5.2 .5 Measurements . . . . . . . . . . . . . . . . . 92 


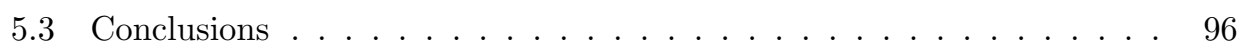

6 Conclusions $\quad 98$

6.1 Summary and Conclusions . . . . . . . . . . . . . . . . . 98

6.2 Original Contributions . . . . . . . . . . . . . . . . . 99

6.3 Future Work ......................... 100

A Switched-RC Kernel Analysis 102

A.1 LPTV Calculation Method . . . . . . . . . . . . . . . . 102

A.2 SE and DI Kernel Calculation . . . . . . . . . . . . . . . . . 105

A.3 Derivation of Kernel Approximations . . . . . . . . . . . . . . . . 108

A.3.1 Mixing Region . . . . . . . . . . . . . . . . . . . . 108

A.3.2 Sampling Region . . . . . . . . . . . . . . . . . 110

A.4 Derivation of Kernel Noise . . . . . . . . . . . . . . . . . . . . . . . 110

A.4.1 Mixing Region .................... . . 111

A.4.2 Sampling Region . . . . . . . . . . . . . . . 111

B Integrals and Sums $\quad 113$

$\begin{array}{ll}\text { Dankwoord } & 115\end{array}$

$\begin{array}{lr}\text { Bibliography } & 117\end{array}$

$\begin{array}{lr}\text { List of Publications } & 123\end{array}$

$\begin{array}{ll}\text { List of Abbreviations } & 125\end{array}$ 


\section{Chapter 1}

\section{Introduction}

\subsection{Wireless Communications}

Imagine throwing a rock in a pond. The impact of the rock will form waves on the surface of the water, traveling away from the location of the splash in circles. Next, consider a fisherman who is fishing somewhere further along the shore of the pond. His floater will oscillate along with the water waves, and he can deduce from this observation that something has disturbed the water some distance away. He can draw two important conclusions.

First, it is apparent that the impact of the waves on the receiver becomes weaker as the distance to the source increases. The energy of the wave is spread across a larger circumference, making it harder to detect the wave. Secondly, from the bobbling of the floater, he cannot tell where the source is located.

Of course, the surface of the water needs to be calm for the fisherman in order to detect the ripples caused by the rock. When a storm is going on, the waves formed by the wind are so large that the smaller ripples are completely obscured. Moreover, if multiple persons are throwing rocks in the pond, the ripples will interfere and make it hard for him to distinguish between them.

Not all water waves behave this way, as can be observed when one sits next to the water front of a lake. Sometimes, a bow wave can be seen coming from no apparent source, striking the water front and reflecting back on the lake. Obviously, it has traveled quite a long distance, as there is no boat in sight and as the wave speeds away again, it does not seem to lose significant amplitude. It seems that the boat causing the bow wave was able to direct its energies towards a specific propagation direction, instead of the circular wave generated by throwing a rock into the water. 
Personal wireless communication devices have secured a firm position in our lives. Whether you are making a phone call to relatives, browsing the web on your laptop through a wireless network (WLAN) or checking the train departure times on your smart phone through a $4 \mathrm{G}$ LTE connection, something is happening inside these devices to establish a connection over large distances.

The primary physical effect that is being exploited is the propagation of waves in the electromagnetic (EM) field. This is analogous to throwing a rock in a pond, where the role of rock and floater is taken up by specially formed pieces of metal, called antenna. As water waves will become weaker with increasing distance, so do EM waves become increasingly harder to detect, putting a maximum range beyond which no reception is possible.

Moreover, the receiver has no direct information about the location of the transmitter. When there are multiple transmitters, their waves will interfere and make it harder for the receiver to detect the desired signal.

The source of the range limitation and interference issues can be found in the omnidirectional (isotropic) characteristics of the antenna in the receiver and transmitter. As the transmit antenna sends its signal in all directions and the receiver listens to all directions simultaneously, a lot of energy is wasted. If the transmitter and receiver could be made more directional, the range would increase and the interference between transmissions would decrease.

In the pond analogy, it was noted that not all water waves are circular, but that bow waves move in a specific direction. The same mechanism can be achieved with electromagnetic waves by transmitting the signal with multiple antennas at the same time. The EM energy can be steered to specific directions, increasing its effectiveness. Moreover, the receiver can also be outfitted with multiple antennas in order to listen to a specific direction, thereby increasing its sensitivity.

The use of multiple-antenna systems is now entering the consumer market in the latest wireless standards. This step promises to increase data rates, reduce coexistence interference and in general improve the quality of service for the consumer. In this thesis, the use of these multi-antenna systems in modern wireless consumer electronics is investigated.

\subsection{CMOS Integrated Circuit Technology}

Aside from the physical principles of wireless systems, there are the electrical devices themselves to consider. It requires a lot of signal processing to generate suitable signals for transmission through the EM medium, as well as for amplifying and decoding the resulting received signal. Moreover, there is all the digital logic needed to interact with the user and provide a rich user experience. The combination of enormous processing 


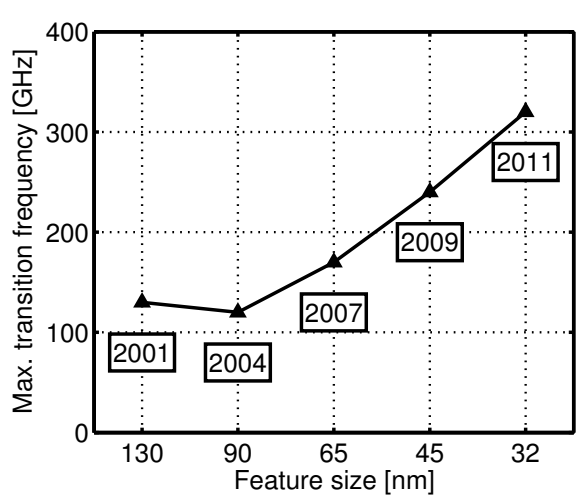

(a)

\begin{tabular}{|c|c|c|}
\hline Component Type & $\begin{array}{l}\text { Compound } \\
\text { Technology }\end{array}$ & $\begin{array}{c}\text { CMOS } \\
\text { Technology }\end{array}$ \\
\hline Microwave & ++ & 一 \\
\hline Inductor $=m$ & + & $+1-$ \\
\hline Capacitor - & + & + \\
\hline$\underset{\text { Transistor }}{R F} K_{4} \|_{-1}$ & + & $+1-$ \\
\hline Logic & 一 & ++ \\
\hline
\end{tabular}

(b)

Figure 1.1: (a) International Technology Roadmap for Semiconductors year of introduction and maximum transition frequency of 130-nm towards 32-nm CMOS [3] and (b) compatibility of component types with compound and CMOS technology.

power in a minuscule form factor has only been possible with the invention of the integrated circuit (IC) by Jack Kilby in 1959 [1].

Instead of assembling electrical circuits component-by-component, the circuits in ICs are constructed all at once, drastically reducing the price per component to fantastically low level. The basic production procedure involves growing patterned layers on top of a semiconductor substrate, thereby forming the components as well as their interconnection. The finished IC is a small silvery slab the size and thickness of a fingernail, but packing up to several billion components [2].

The inclusion of more and more functionality on a single IC (or chip) is known as integration. Two major substrate types are in use for the fabrication of ICs, with different trade-offs in integration and performance. First of all, there are the technologies using a compound between III and V materials in the periodic table. Examples are Gallium-Arsenide (GaAs), Indium-Phosphide (InP) and Gallium-Nitride (GaN). These offer the best performance, at the expense of integration and at a rather high cost, and therefore find uses mostly in the defense and scientific sector.

On the other hand, there are the technologies based on silicon, a IV material, literally as abundant as sand (which is silicon dioxide). Aside from Silicon-Germanium ( $\mathrm{SiGe}$, a cheaper alternative for the compound materials, there is the highly specialized silicon complementary metal oxide semiconductor (CMOS) technology. CMOS is the main driver behind the consumer market, as it is very cheap to produce in mass volumes and can achieve the highest integration, at the expense of performance.

CMOS is first and foremost developed for a high integration of digital electronics, like processors and memories. Scaling is the key word here [4], the minimum compo- 
nent size (called feature size) steadily decreases from year to year, as is illustrated in Fig. 1.1a. Hence, a doubling of the integrated functionality on the same chip area for every two years is achieved. This is perceived by the consumer as an increase in storage capacity and computing power that is available in electronic products every year.

But, wireless transmitters and receivers are analog circuits and follow different scaling rules than digital circuits. The decrease in transistor dimensions that is so beneficial for digital integration has the effect of higher transition frequencies $f_{T}$ for analog transconductor circuits. This transition frequency is a measure for the maximum frequency for which meaningful amplification can be reached, indicating that newer nodes can process higher frequency signals.

The improved switching speeds in advanced CMOS open up new communication bands which where only reachable with compound technologies before. Costs for mass produced products can be lowered by moving to CMOS, opening up these bands for the broad public.

For the circuit designer, moving circuits from compound technologies to CMOS can be challenging. Fig. $1.1 \mathrm{~b}$ indicates the compatibility of components types with the two technology families. Where as in compound technology the use of microwave structures and inductors is beneficial for high frequency circuits, in CMOS these components are more difficult to integrate. On the other hand, the performance of switched circuits, derived from digital logic, in CMOS is increasing. It is up to the circuit designer to develop circuits utilizing the components that scale best in newer technologies.

\subsection{Multi-Antenna Wireless Systems}

As was sketched in the pond analogy, there is an advantage in expanding our wireless systems to use multiple antennas. Instead of exciting and probing the electromagnetic field in a singular point location ${ }^{1}$, extra degrees of freedom are created by placing multiple point source some distance (in the order of the carrier wavelength) apart. The techniques involving multiple antennas are referred to as multiple-input-multipleoutput (MIMO) systems and come in several varieties.

First, consider the highly scattering environment in Fig. 1.2a. From each transmitter to each receiver antenna, there are multiple propagation paths possible due to the occurrence of reflecting objects. Traditionally, these multiple paths were used to transmit the same signal, increasing spatial diversity and therefore adding robustness in a fading environment. It has been understood however that the fading can actually be beneficial, as it creates parallel communication paths. This is used for

\footnotetext{
${ }^{1}$ We stick here to the unrealizable, but insightful notion of an isotropic point source.
} 


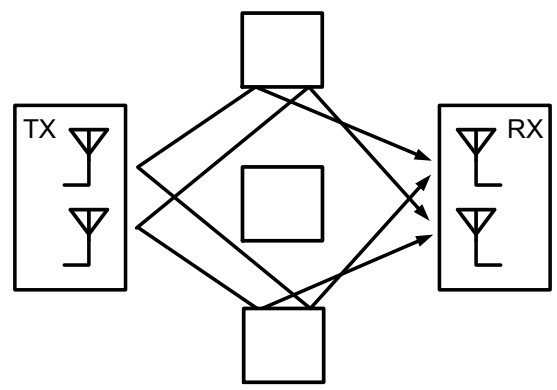

(a)

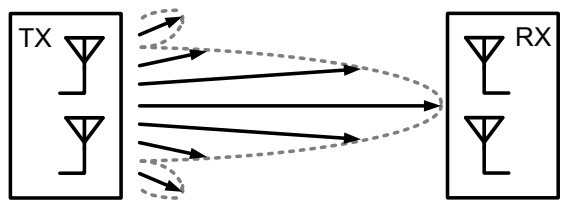

(b)

Figure 1.2: MIMO systems employing (a) spatial multiplexing and (b) beamforming.

spatial multiplexing, where multiple data streams are transmitted over orthogonal transmit-receive paths [5], thereby increasing the capacity of the channel.

In a low scattering (line-of-sight) environment, Fig. 1.2b, the advantages of spatial multiplexing are less as there is a lower orthogonality between receiver and transmitter antenna pairs. Here, the high correlation between propagation paths can be used to direct EM energy into specific directions. This process is known as beamforming, as the signals are formed into a beam pointed at the receivers. The overall sensitivity of the system is increased and the receiver becomes less sensitive to interferers with a different angular position than the transmitter, as it is listening from a specific direction.

The support for MIMO systems in consumer electronics has started only recently. For WLAN, the IEEE 802.11n amendment defines transmit beamforming [6]. In cellular systems, LTE release 9 defines several MIMO modes, including spatial multiplexing, downlink transmitter beamforming and a combination of the two [7]. Note that these standards support full transmitter-receiver spatial multiplexing, but only partial (transmitter) beamforming. From the receiver side, applying beamforming has the potential to boost sensitivity and reject interferers [8].

Looking at the receiver block schematic of a typical MIMO system, Fig. 1.3a, there is a distinctive subdivision between the elements of the receiver chain. First, electronic circuits in the analog domain amplify and filter the incoming signal from each antenna. These circuits are grouped together to form the analog front end. Then, an analog-to-digital converter (ADC) converts the signal to the digital domain, after which the digital processing performs the MIMO computation and decoding.

It is critical to realize that up to the digital processing, the receiver chain is identical to the one employed in a single-antenna system, and is therefore susceptible to interferers. Especially the ADC is vulnerable, as it has the most stringent requirements on dynamic range. Placing the beamforming operation in the analog domain, 


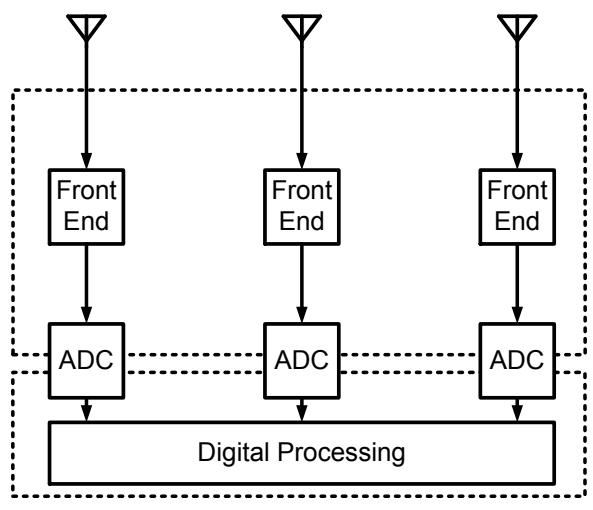

(a)

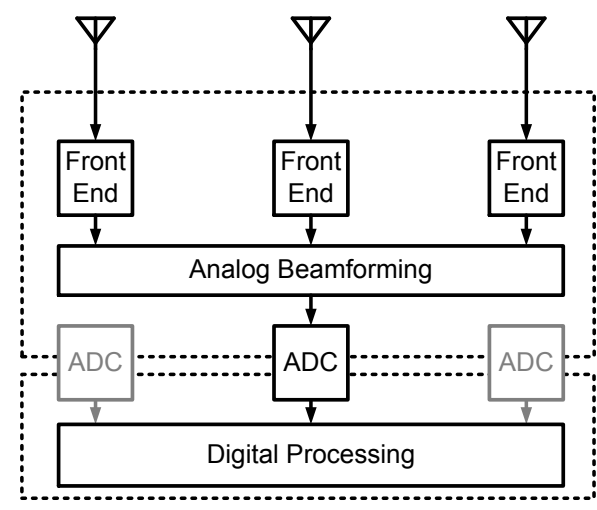

(b)

Figure 1.3: (a) Digital-MIMO receiver and (b) proposed analog adaptive beamforming receiver.

as close toward the antennas as possible, enables the rejection of interferers early up in the chain and allows the disabling of some of the power-hungry ADCs.

By designing an adaptive receiver that can switch between digital spatial multiplexing and analog beamforming, it becomes possible to allow high data rates under favorable conditions in the spatial multiplexing mode, and extended range and interferer robustness under harsh conditions in the beamforming mode.

Analog beamforming has been extensively studied and implemented in phased array radars. Here, a very large number of antenna elements (thousands) is used to send EM energy into a narrow beam towards a target, which reflection is picked up by the same phased array network, creating a high sensitivity in that particular direction and a large suppression towards other directions. These systems are closest to the bow wave sketched in the pond analogy and display the absolute best that can be achieved with beamforming.

The big advantage of these radars compared to mechanically rotating reflectorbased radars is their much faster electronic steering of the beam. Antenna arrays with fixed beams were being used in the early days of radio astronomy [9] and transatlantic radio communication [10]. The second world war saw use of fixed phased-array radars with mechanically adjusted phase shifters by the United States, Great Brittain and Germany [11]. The development of ferrite and diode-switched phase shifters [12] [13] led to the first truly electronically steered array system in 1978 [14].

With advances in monolithic microwave integrated circuits (MMIC) in compound technologies, it became possible to build active phased arrays with a transmit/receive (T/R) module for each antenna element [15]. Each T/R module has amplifiers, phase shifters and filters, preferably integrated on to a single die [16] [17] [18]. These modules 
were cheaper to manufacture and had smaller dimensions than their ferrite counterparts, enabling a tight system integration.

In the new millennium, focus has shifted to the integration of multiple elements on a single die, to further reduce the cost and size. Advances on radar systems in the 8-12 $\mathrm{GHz}$ [19], $24 \mathrm{GHz}$ [20] and $77 \mathrm{GHz}$ [21] [22] frequency band open up the introduction of phased-array radars in consumer electronics, for example in automotive radar.

With this shift to the consumer market, initiative is taken to convert the bipolar designs in compound and SiGe technologies to CMOS . Fully integrated phased arrays in the $24 \mathrm{GHz}$ band for radar [23] and the $60 \mathrm{GHz}$ band for wireless communication [24] [25] [26] have been demonstrated. But, as was outlined in Fig. 1.1b, CMOS has different strengths and weaknesses compared to the other technologies. This indicates that new circuit topologies, specifically designed for CMOS, might be able to outperform direct ports from compound technologies.

\subsection{Motivation and Thesis Outline}

The addition of analog beamforming in CMOS MIMO receivers has the potential of increasing its interferer robustness. This spatial filtering of interferers can become very attractive in the coming years, with decreasing supply voltages in new CMOS technology nodes and its associated decrease in circuit linearity [27], together with the increased co-existence problem due to the growing number of wireless nodes.

Over the past 50 years, much experience on analog beamforming has been gained in the field of phased-array radars. However, the difference in circuit strengths and weaknesses between compound and CMOS technology requires the design of new circuit topologies, which are inherently suitable for CMOS integration.

The rest of the thesis is organized as follows. First, chapter 2 analyzes the systemlevel properties of beamforming systems. Basic phased-array properties are derived and the effect of errors in the array is analyzed. The inclusion of downconversion in the receiver chain is modeled and transformation methods for relocating beamforming to the RF, LO and IF domain are presented.

Next, chapter 3 explores highly linear downconversion techniques, for use in interference robust receivers. To this end, the linear mixer-first receiver concept is introduced. A unified analysis of polyphase switched-RC samplers and mixers is made, and the derived results are demonstrated in a mixer-first receiver.

In chapter 4 , the concept of a phase shifter operating in the discrete-time domain is proposed. It is shown that a rational approximation of the sine and cosine function can adequately apply the correct weighting in a vector modulator phase shifter, while being easily mapped on a switched-capacitor circuit implementation. An implementation of a 4-element beamforming receiver utilizing this phase shifter achieves 
interference suppression through a null steering algorithm.

Chapter 5 further expands the switched-RC circuit theory to include coupled loops. With this new design methodology, the switched capacitor highly-linear downconverter from chapter 3 can be coupled to the high accuracy switched-capacitor phase shifter from chapter 4, resulting in a fully-passive beamforming architecture. The implementation achieves high dynamic range and is easily integrated in advanced CMOS.

Finally, Chapter 6 presents the summary and conclusions, along with directions along which to take future research. 


\section{Chapter 2}

\section{Phased-Array Downconverting Receivers}

This chapter explores the fundamental properties of phased-array downconverting receivers, in particular for 1-dimensional linear arrays employing analogue beamforming in the presence of imperfections.

Furthermore, a transformation method for arrays with frequency downconversion is derived, allowing the relocation of beamforming to the RF, LO and IF domain.

\subsection{Linear Array Theory}

The basic antenna configuration of a phased array is composed by multiple antennas positioned on a line, Fig. 2.1a, with uniform spacing $d^{1}$. The direction perpendicular to this line referred to as the broadside direction and the direction of the line itself the endfire direction. It is assumed that the distance from the transmitter is large enough, such that the incoming electromagnetic wavefront can be considered a plane wave.

Because of the physical separation between antennas, a plane wave with directionof-arrival $\theta$ will have a different traveling path to each antenna. Having a total of $N$ antennas and numbering each antenna from $n=0$ at the top towards $n=N-1$ at the bottom, the extra traveling length $l_{n}$ for the $n$-th antenna can be expressed as:

$$
l_{n}=n \cdot d \sin (\theta) .
$$

As the wave travels at the speed of light $c$, the delay after which a wavefront reaches

\footnotetext{
${ }^{1}$ Two-dimensional arrays are not considered, but share many of the same properties.
} 


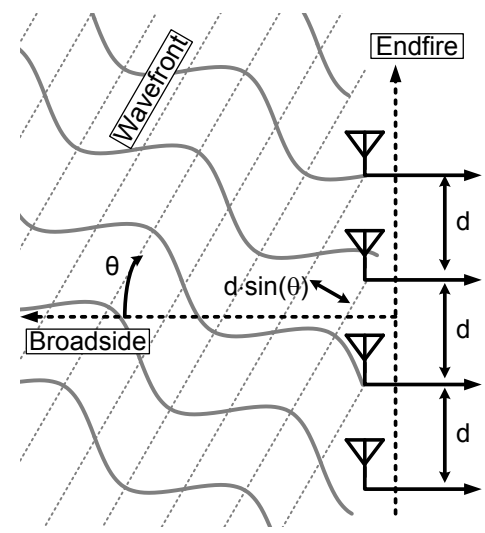

(a)

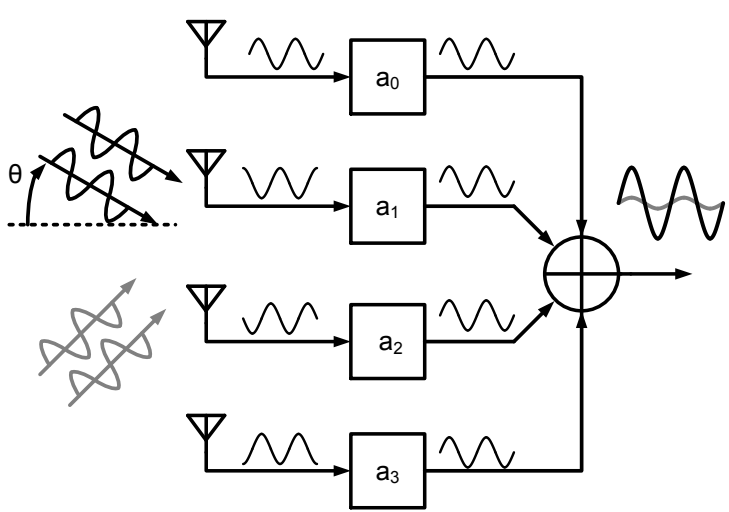

(b)

Figure 2.1: Example of (a) the incoming wavefronts and (b) the antenna combining system of a 4-element phased array.

an antenna is:

$$
\tau_{n}=\frac{l_{n}}{c}=n \cdot \frac{d}{c} \sin (\theta) .
$$

Representing the received signal with amplitude $A$ and carrier frequency $f$ at the topmost antenna in its phasor form:

$$
s_{0}(t)=A \cos (2 \pi f t)=\operatorname{Re}\left\{A \cdot e^{j 2 \pi f t}\right\},
$$

the received signal at the other antennas can be represented by:

$$
\begin{aligned}
s_{n}(t) & =\operatorname{Re}\left\{A \cdot e^{j 2 \pi f t} \cdot e^{j 2 \pi f \cdot n \frac{d}{c} \sin (\theta)}\right\} \\
& =\operatorname{Re}\left\{A \cdot e^{j 2 \pi f t} \cdot e^{j 2 \pi n \cdot \frac{d}{\lambda} u}\right\}
\end{aligned}
$$

where $\lambda=\frac{c}{f}$ is the wavelength of the carrier frequency and $u$ is defined as $\sin (\theta)$. Hence, the different time-of-arrival between antennas expresses itself as an extra phase term in the signal phasor which increases linearly with frequency.

The fundamental theories behind phased arrays was developed in the 1960s [28]. In this section, a summary is given of these results.

\subsubsection{Beam Steering}

To enable steering of the array, the conceptual circuit in the block diagram in Fig. $2.1 \mathrm{~b}$ is used. A circuit block with transfer function (or weight) $a_{n}$ is inserted after each antenna. The amplitude characteristics of the weights are referred to as the tapering of the array and for now will be assumed to be equal to one (uniform taper). 


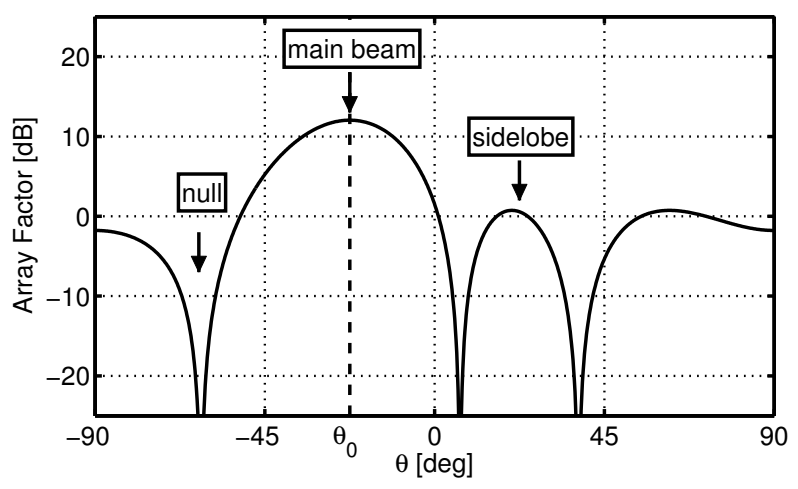

Figure 2.2: Array factor for a 4-element phased array.

In the first step of beam steering, the phase responses of the weights are set to the inverse time delays of their respective antennas. The weights to accomplish this are given by:

$$
a_{n}=e^{-j 2 \pi \cdot n \frac{d}{\lambda} u_{0}},
$$

where $u_{0}=\sin \left(\theta_{0}\right)$, the sine of the direction-of-arrival of the incoming wavefront. This has the effect of countering the delay between antennas for a specific directionof-arrival $\theta_{0}$, such that the received signals are aligned in time. Next, a summing node is introduced that sums all element outputs together. Its output signal can be written as:

$$
s_{\text {out }}(t)=\operatorname{Re}\left\{s_{0}(t) \cdot \sum_{n=0}^{N-1} a_{n} \cdot e^{j 2 \pi \cdot n \frac{d}{\lambda} u}\right\} .
$$

The output is maximum for direction $\theta_{0}$, but smaller or even zero for other directions.

The contribution due to the summing over the elements is called the array factor $A F$ and constitutes the gain of the signal with respect to a single antenna as a function of the direction-of-arrival [29]. Inserted the weights from (2.5) into the summing equation (2.6) gives the array factor as:

$$
A F(u)=\sum_{n=0}^{N-1} e^{j 2 \pi \cdot n \frac{d}{\lambda}\left(u-u_{0}\right)} .
$$

Fig. 2.2 shows an example of the array factor for a 4 -element phased array. The plot has one absolute maximum, which is defined as the main beam. Lower local maxima occur and are referred to as sidelobes, while completely destructive summing occurs at the nulls. Hence, the array factor describes the spatial filtering that is performed by the array.

Equation 2.7 shows that the array factor is a function of $\left(u-u_{0}\right)$, so that if a pattern is steered to an angle, the pattern remains unchanged except for a translation. For this 


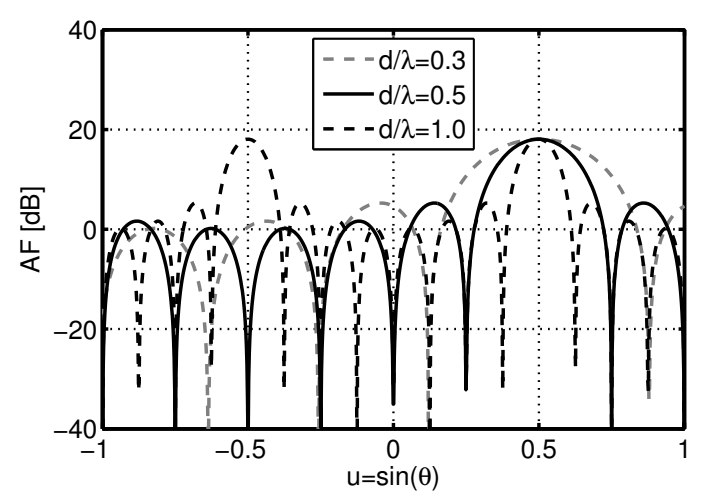

(a)

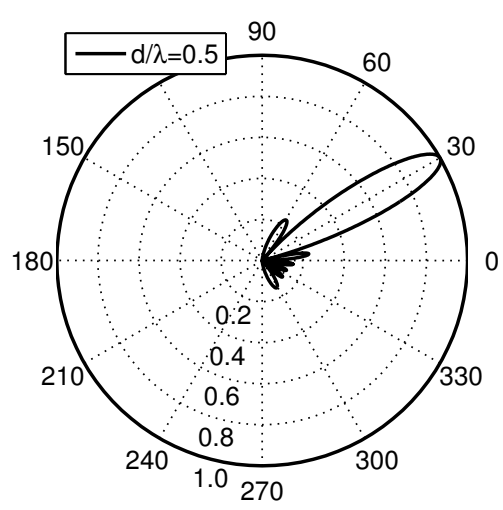

(b)

Figure 2.3: Array factor for an 8-element phased array steered to $u_{0}=0.5$.

reason the variables $u$ and $u_{0}$ are used, in sine space [30]. A closed form expression for the array factor of a uniformly illuminated array $\left(\left|a_{n}\right|=1\right)$ steered to direction $u_{0}$ can be found [30]:

$$
A F_{\text {uni }, u_{0}}(u)=\underbrace{\frac{\sin \left[N \cdot \pi \frac{d}{\lambda}\left(u-u_{0}\right)\right]}{\sin \left[\pi \frac{d}{\lambda}\left(u-u_{0}\right)\right]}}_{\text {Amplitude }} \cdot \underbrace{e^{j \pi \frac{d}{\lambda}(N-1) \cdot\left(u-u_{0}\right)}}_{\text {Phase }} .
$$

Fig. 2.3 plots this antenna factor for several values of $d / \lambda$. As the antenna spacing with respect to the wavelength increases, the width of the main beam decreases due to the increased effective length $L$ of the array:

$$
L=N \cdot d
$$

The half-power or $-3 \mathrm{~dB}$ width of the beam can be calculated to be [31]:

$$
u_{-3 d B}=0.886 \frac{\lambda}{L} \text {. }
$$

For achieving the narrowest beam width, it is therefore essential to increase the antenna spacing as much as possible. However, there is a point where additional main beams, the grating lobes, occur in directions other than $\theta_{0}$. These beams are positioned at locations [32]:

$$
u=u_{0}+i \cdot \frac{\lambda}{d}, \quad i=. .,-2,-1,0,1,2, . .
$$

So that in order to avoid grating lobes, the maximum antenna spacing for a full scan range is:

$$
\frac{d}{\lambda_{\min }} \leq \frac{1}{2}
$$




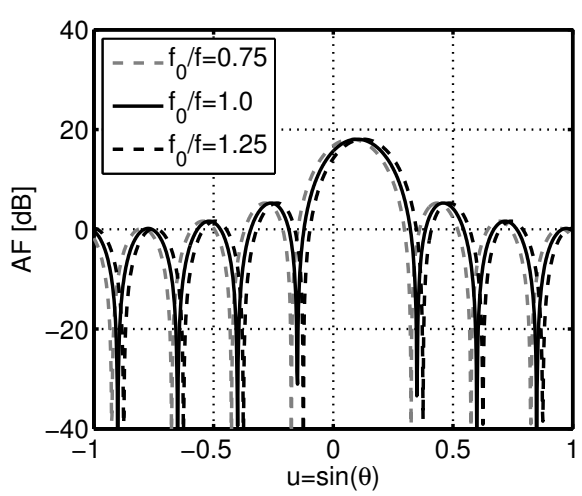

(a)

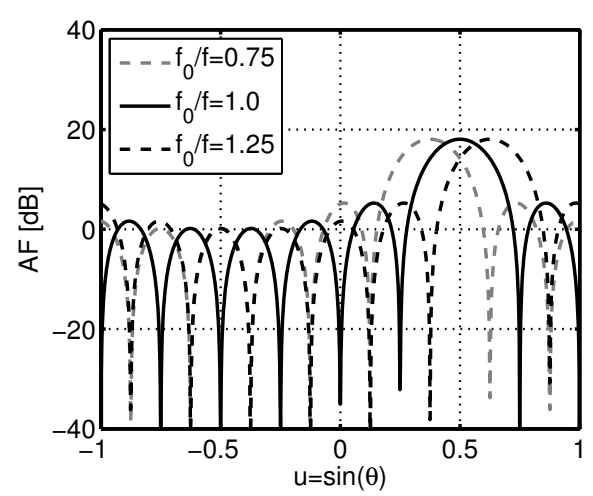

(b)

Figure 2.4: Beam squint in an 8-element phase shifter steered array for (a) $u_{0}=0.1$ and (b) $u_{0}=0.5$.

where $\lambda_{\min }$ is the wavelength of the maximum frequency of interest. This criterium has an analogy with the Nyquist sampling theorem, in that the signal has to be sampled at least at half its wavelength along the aperture.

From here on the distance between the antennas is chosen such that the spacing is half the wavelength at the highest frequency, $f_{0}$, resulting in an array factor

$$
A F(u)=\sum_{n=0}^{N-1} e^{j \pi \cdot n \frac{f}{f_{0}}\left(u-u_{0}\right)} .
$$

\subsubsection{Narrowband Phase Shifter Approximation}

From equation (2.5) we found that in order to steer the beam, time delays are required at each of the antenna elements. If the array is to operate only in a narrow frequency band around frequency $f_{0}$ (for which there is half a wavelength of spacing), the time delay can be approximated with a phase shift, which can be easier to implement.

To this end, the conditions $\lambda=1 / f_{0}$ and $d=\lambda / 2$ are applied to weight equation with time delays (2.5). The new weights in this case are:

$$
a_{n}=e^{-j \pi n \cdot u_{0}}
$$

Inserting into the array factor equation (2.6) gives:

$$
A F(u)=\sum_{n=0}^{N-1} e^{j \pi \cdot n \frac{f}{f_{0}}\left(u-\frac{f_{0}}{f} u_{0}\right)} .
$$


Defining the frequency deviation $\Delta f=f_{0}-f$, this can be rewritten as:

$$
A F(u)=\sum_{n=0}^{N-1} e^{j \pi \cdot n \frac{f}{f_{0}}\left(u-\left(1+\frac{\Delta f}{f}\right) u_{0}\right)}=\sum_{n=0}^{N-1} e^{j \pi \cdot n \frac{f}{f_{0}}\left(u-u_{0}\right)} \cdot \underbrace{e^{-j \pi \cdot n \frac{\Delta f}{f_{0}} u_{0}}}_{\text {Excess phase }} .
$$

Compared to the array factor with time delays (2.13), the location of the main beam is now frequency dependent:

$$
u_{0}^{\prime}(f)=\left(1+\frac{\Delta f}{f}\right) u_{0}
$$

as a result of the excess phase in (2.16) [33]. Defining the shift in location as $\Delta u(f)$ :

$$
u_{0}^{\prime}(f)=u_{0}+\Delta u \quad \Rightarrow \quad \frac{\Delta u(f)}{u_{0}}=\frac{\Delta f}{f} .
$$

Or stated in words, the fractional shift in main beam position is equal to the fractional shift in frequency [30]. This behavior is known as beam squint. It increases for larger steering angles and for larger frequency differences, as is illustrated in Fig. 2.4. When considered in a single direction, the gain of the array is now frequency dependent, with $3 \mathrm{~dB}$ attenuation at the point where $\Delta u(f)=u_{-3 d B}$ (applying equation (2.10)). Therefore, the bandwidth of a phased array steered with phase shifters is inherently limited, regardless of the electrical bandwidth of the circuits:

$$
B W_{-3 d B}=f \frac{u_{-3 d B}}{u_{0}} \approx f_{0} \frac{2}{u_{0} N} .
$$

The fractional bandwidth for a full scan range is thus inversely proportional to the number of antennas.

\subsubsection{Directivity}

It can be shown that the element weights and array factor form the Fourier series pair $[30]$ :

$$
\begin{gathered}
a_{n}=\frac{1}{2} \int_{-1}^{1} A F(u) \cdot e^{-j \pi \cdot n \cdot u} d u \\
A F(u)=\sum_{n=0}^{N-1} a_{n} \cdot e^{j \pi \cdot n \cdot u} .
\end{gathered}
$$

For such a pair, the power in one domain $(A F(u))$ is equal to the power in the other domain $\left(a_{n}\right)$, so that Parseval's theorem can be applied:

$$
\sum_{n=0}^{N-1}\left|a_{n}\right|^{2}=\int_{-1}^{1}|A F(u)|^{2} d u
$$




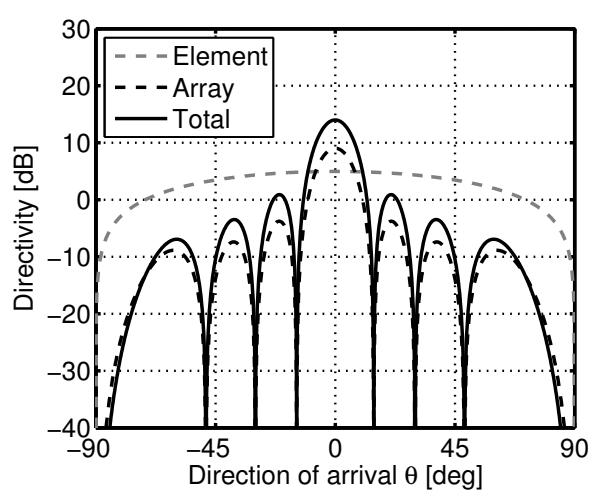

(a)

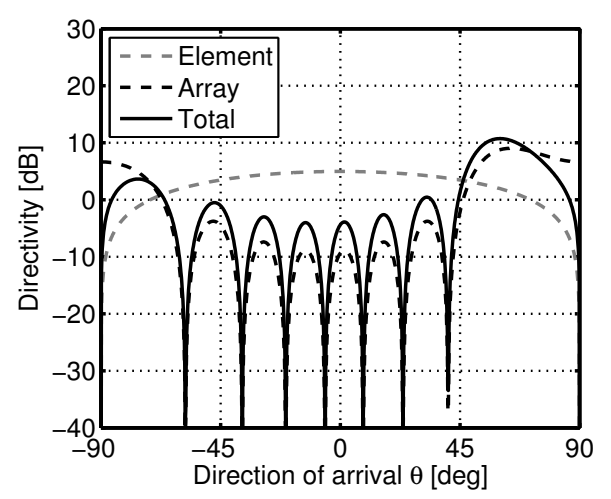

(b)

Figure 2.5: Antenna element, array and total directivity for beam steered (a) to broadside and (b) close to endfire.

The directivity $D$ is defined as the ratio between the radiation intensity of a nonisotropic source in a given direction over that of an isotropic source with the same power[29]. It is a fundamental quality of the antenna pattern, because it is derived from just the beam shape.

A useful insight can be obtained by applying the conservation of energy. Then, the integral of the directivity over all space must be equal to one. For a phased array with a +90 to -90 degrees scanning angle, this amounts to:

$$
\int_{-\pi}^{\pi} D(\theta) d \theta=1
$$

Intuitively, the array factor is connected to the directivity. Applying the integral substitution rule to equation (2.22) for $u=\sin (\theta)$ brings the integral to the angular domain:

$$
\begin{gathered}
\int_{-1}^{1}|A F(u)|^{2} d u=\int_{-\pi}^{\pi} \cos (\theta) \cdot|A F(\theta)|^{2} d \theta=\sum_{n=0}^{N-1}\left|a_{n}\right|^{2} \\
\int_{-\pi}^{\pi}[\underbrace{\cos (\theta)}_{D_{E}} \cdot \underbrace{|A F(\theta)|^{2} / \sum_{n=0}^{N-1}\left|a_{n}\right|^{2}}_{D_{A}}] d \theta=1 \\
\Rightarrow D=D_{E} \cdot D_{A} .
\end{gathered}
$$

Comparing with equation (2.23), the directivity of the total system has two components. The contribution due to the array factor is normalized by the summed power in the weights and is defined as the array directivity $D_{A}$. The second contribution is 


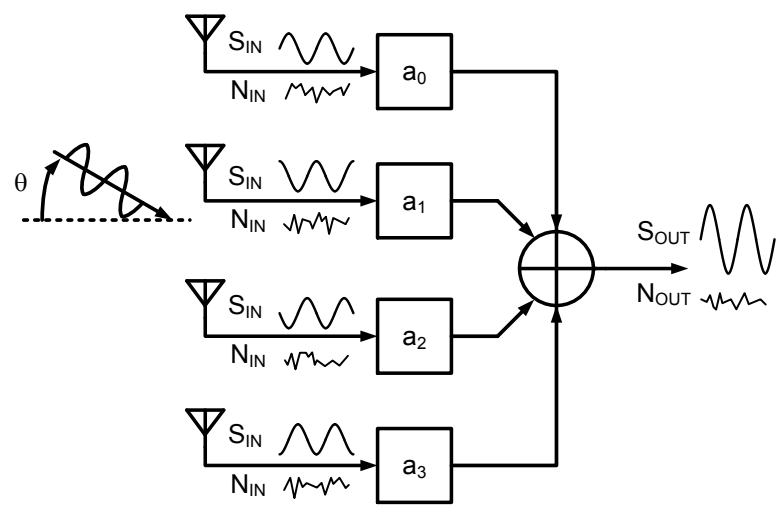

Figure 2.6: Correlated signals and uncorrelated noise in a phased-array system.

the directivity of an element in the array and arises because of the arrangement of the elements on a line and the projection of the incoming EM wave on that line.

These two contributions and the total directivity are plotted in Fig. 2.5 with the beam scanned to broadside and towards end fire. As the beam is scanned to end fire, the total directivity in the main beam decreases and the width of the beam increases. From the EM wave source point of view, this is because it sees a shorter effective length that captures less EM energy due to the angle of arrival. Therefore, even though the analysis started by assuming isotropic elements, energy conservation dictates that when these elements are put into an array, the element directivity is changed to $\cos (\theta)[29][31]$.

So what does directivity mean to the circuit designer? Concentrating on the array factor and assuming isotropic element factors, consider the array in Fig. 2.6. Each input receives signal power $S_{i n}$. It is important to note that the signals are fully correlated between elements, regardless of their phase differences. The output signal is related to the array factor, such that:

$$
S_{\text {out }}(\theta)=S_{\text {in }} \cdot|A F(\theta)|^{2}
$$

Now consider the noise right after the antenna element. It consists of the inputreferred noise of the element electronic circuits and the antenna EM noise. The antenna noise finds its origin in the perturbations in the electromagnetic field, which for reasons of simplicity is assumed here to have a uniform noise temperature. The noise powers between antennas are fully uncorrelated ${ }^{2}$. As a result, the powers are always summed in the same manner in the summing node, regardless of the intervening

\footnotetext{
${ }^{2}$ It is assumed that there is no mutual coupling.
} 
phase shifts and it can be stated that for all angles:

$$
N_{\text {out }}=N_{\text {in }} \cdot \sum_{n=0}^{N-1}\left|a_{n}\right|^{2} .
$$

If the ratio between signal power and noise power is taken:

$$
\frac{S_{\text {out }}}{N_{\text {out }}}(\theta)=\frac{S_{\text {in }}}{N_{\text {in }}} \cdot|A F(\theta)|^{2} / \sum_{n=0}^{N-1}\left|a_{n}\right|^{2}=\frac{S_{\text {in }}}{N_{\text {in }}} \cdot D_{A},
$$

it is found that directivity is the improvement in signal-to-noise ratio (SNR) compared to a single isotropic antenna due to the directional properties of the aperture. In a similar way, it can be proven that the same property holds for the element directivity and for any other antenna aperture.

In publications, the element factor is usually not taken into account, because it is fundamentally the same for all phased arrays, and only the array directivity is reported. The maximum in array directivity can be found in the beam pointing direction, where [31]:

$$
\max \left(D_{A}\right)=\left|A F\left(\theta_{0}\right)\right|^{2} / \sum_{n=0}^{N-1}\left|a_{n}\right|^{2}=\left(\sum_{n=0}^{N-1}\left|a_{n}\right|\right)^{2} / \sum_{n=0}^{N-1}\left|a_{n}\right|^{2} \equiv \epsilon_{T} \cdot N,
$$

where $\epsilon_{T}$ is the taper efficiency (and equal to one for uniform tapering). In other words, the maximum directivity occurs for a uniform taper and is equal to $10 \cdot \log (N)$ dB.

\subsubsection{Amplitude Tapering}

Although a uniform tapering yields maximum directivity (and therefore minimum beam width), there is an advantage to choosing a different amplitude distribution over the antenna elements which has sparked significant research attention [34][35]. This advantage is the ability to manipulate the sidelobe level relative to the main beam and thus provide better spatial filtering outside of the main beam. Most tapers have a parameter which controls the amount of sidelobe reduction.

Figure 2.7a plots the array factor for a uniform, Gaussian and Taylor taper with the associated amplitude distribution in Fig. 2.7b. Indeed, the sidelobe level is reduced at the cost of increased beam width. The equation of the half-power or $-3 \mathrm{~dB}$ width of the beam (2.10) can be modified to include this effect [30]:

$$
u_{-3 d B}=0.886 \cdot B_{b} \frac{\lambda}{L},
$$

where $B_{b}$ is the beam broadening factor, equal to unity for the uniformly illuminated array. From equation 2.30 the loss in directivity with respect to a uniform taper was 


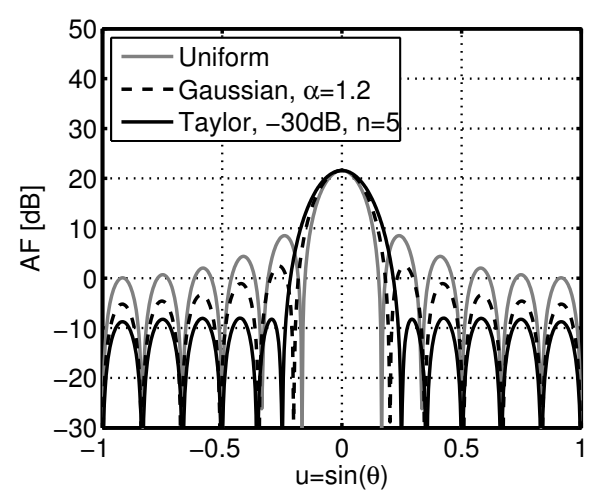

(a)

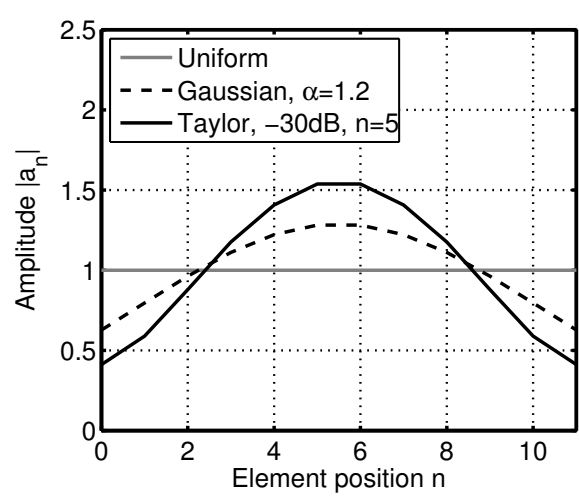

(b)

Figure 2.7: 12-element (a) array factor and (b) amplitude distribution for three amplitude tapers.

expressed in the taper efficiency $\epsilon_{T}$, which can be rewritten as:

$$
\frac{1}{\epsilon_{T}}=1+\frac{1}{N} \sum\left(\frac{\left|a_{n}\right|}{\text { mean }\left|a_{n}\right|}-1\right)^{2} .
$$

This equation states that the taper efficiency is always smaller than one and worsens when the amplitudes deviate more from the mean amplitude. For the specific Gaussian and Taylor tapers shown in the picture, the taper efficiency is -0.2 and $-0.7 \mathrm{~dB}$ respectively, with a beam broadening factor of 1.12 and 1.26 respectively.

The choice for a specific taper type is determined by its particular properties. For example, a Chebychev taper has equal sidelobe levels but has an impractical amplitude distribution for large arrays (which are mitigated in the derived Taylor taper), while the Gaussian taper provides the optimum balance between beam width and overall sidelobe level ${ }^{3}$. Amplitude tapering is most effective for large arrays which have pencil beams and where the sidelobes dominate the array factor.

\subsubsection{Null Steering}

In a receiver environment with few antenna elements, amplitude tapering is less effective, as the main beam will widen significantly. For example, in a 4 element array, the two sidelobes are $-10 \mathrm{~dB}$ below the main beam, resulting in a modest rejection.

However, in the case of a single dominant interferer, the rejection can be significantly increased if a null would be adaptively steered on its direction. It is assumed that the direction of the interferer is known a-priori or is determined by an additive

\footnotetext{
${ }^{3}$ The uniform taper is just a special case of the Gaussian taper with $\alpha=0$.
} 


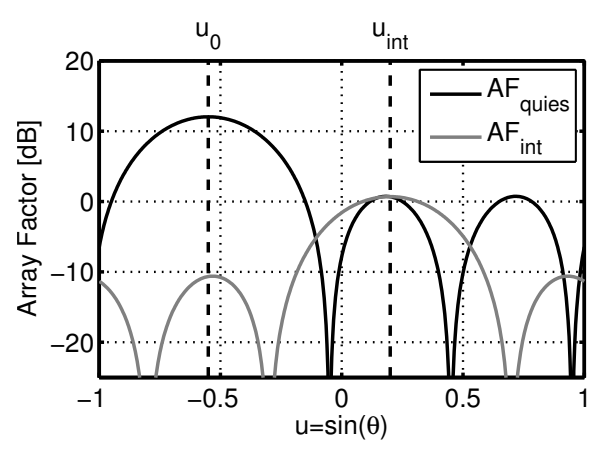

(a)

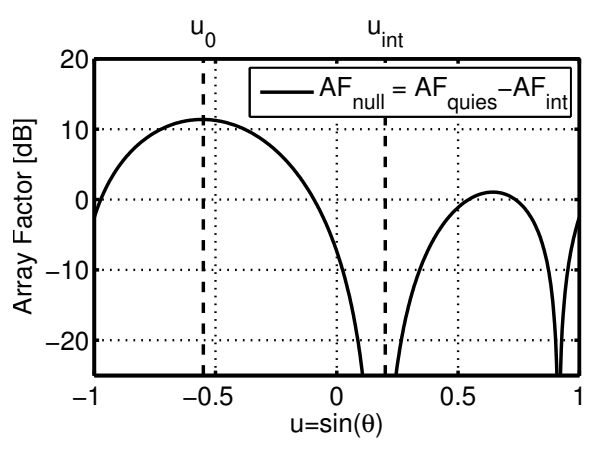

(b)

Figure 2.8: Subtraction of (a) interferer pattern $A F_{\text {int }}$ from quiescent pattern $A F_{\text {quies }}$ results in (b) an array factor with a null on the interferer position.

steering algorithm. From the beamforming properties analyzed in this chapter so far, it is clear that the position of the nulls is rather fixed with respect to the main beam. Therefore, a different kind of pattern synthesis is required.

A graphical representation of the beam pattern synthesis to enable null steering is illustrated in Fig. 2.8a [36]. The goal is to adapt the regular beam pattern $A F_{\text {quies }}$, steered towards the location of the signal-of-interest $u_{0}$, such that it has a null on the location of the interferer, $u_{\text {int }}$.

In order to do so, we rely on the linear properties of the array factor, i.e. a linear combination of array factors is equal to a single array factor with the same linear combination of the weights. If two array factors can be created such that their subtraction creates a null on the desired location, then the subtraction of the weights for these array factors will result in new weights for a single array factor with the same nulling.

The cancellation array factor, $A F_{\text {int }}$, is introduced with main beam at the location of the interferer, $u_{\text {int }}$, and scaled to the height of the quiescent array factor at $u_{\text {int }}$. Therefore, subtraction of the quiescent and cancellation array factor will result in a new array factor with a null at the interferer location, as shown in Fig. 2.8b. This new array factor is:

$$
A F_{\text {null }}(u)=A F_{\text {quies }}(u)-A F_{\text {int }}(u)=A F_{\text {uni }, u_{0}}(u)-A F_{\text {uni }, u_{0}}\left(u_{\text {int }}\right) \cdot \frac{1}{N} A F_{\text {uni }, u_{\text {int }}}(u)
$$

Inserting the array factor for uniform tapering (2.8):

$$
A F_{\text {null }}(u)=\sum_{n=0}^{N-1} e^{j \pi n\left(u-u_{0}\right)}-\frac{\sin \left[N \cdot \frac{\pi}{2}\left(u_{\text {int }}-u_{0}\right)\right]}{N \cdot \sin \left[\frac{\pi}{2}\left(u_{\text {int }}-u_{0}\right)\right]} e^{j \pi \frac{N-1}{2}\left(u_{\text {int }}-u_{0}\right)} \cdot \sum_{n=0}^{N-1} e^{j \pi n\left(u-u_{\text {int }}\right)}
$$




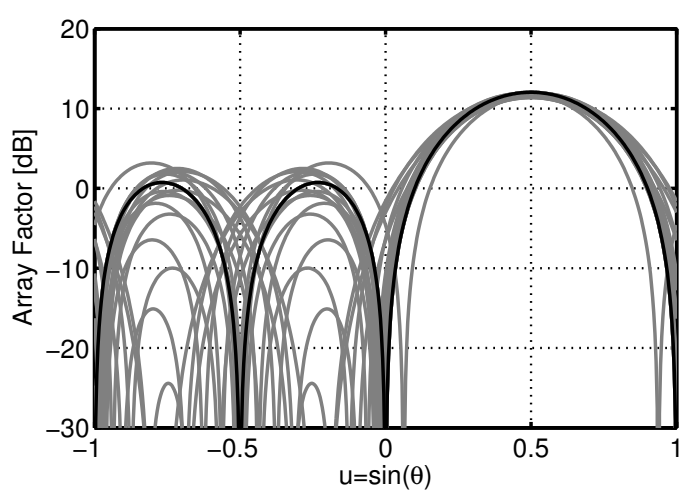

(a)

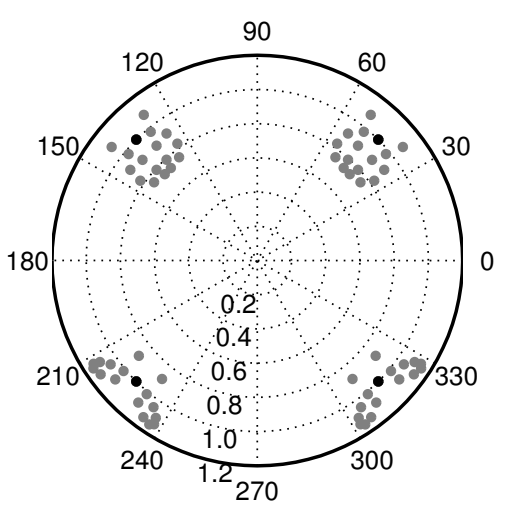

(b)

Figure 2.9: Adaptive nulling (a) array factors and (b) element weights with main beam at $u=0.5$ and interferer direction swept from $u=-1$ to $u=0.1$.

And some reordering gives the total array factor as:

$A F_{\text {null }}(u)=\sum_{n=0}^{N-1} \underbrace{\left(e^{-j \pi n \cdot u_{0}}-\frac{\sin \left[N \cdot \frac{\pi}{2}\left(u_{\mathrm{int}}-u_{0}\right)\right]}{N \cdot \sin \left[\frac{\pi}{2}\left(u_{\mathrm{int}}-u_{0}\right)\right]} \cdot e^{j \pi\left(\frac{N-1}{2} \cdot\left(u_{\mathrm{int}}-u_{0}\right)-n \cdot u_{\mathrm{int}}\right)}\right)}_{a_{n}} \cdot e^{j \pi n u}$

From Fig. 2.8a, it can be deduced that the main beam remains largely unaffected as long as the interferer has a sufficiently different angle-of-arrival, as the main beam is in the sidelobe of the cancellation pattern. In a worst case scenario the interferer is at a quiescent pattern sidelobe, being scaled $10 \mathrm{~dB}$ below the main beam to ensure nulling. At the main beam, the cancellation sidelobe is another $10 \mathrm{~dB}$ lower, resulting after subtraction in at most a modest $-1 \mathrm{~dB}$ gain loss. This is illustrated in Fig. 2.9a, which plots the array factors of the adaptive nulling algorithm for different interferer directions.

Similarly, from (2.35) it can be deduced that the resulting element weights for null steering are a small perturbation of the original weights for beam steering (Fig. 2.9b). The scaling of the cancellation pattern results in the second part of the subtraction having an amplitude between zero and one-third, whereas the first part of the subtraction has unity amplitude. Therefore, the resulting amplitudes $\left|a_{n}\right|$ are close to unity themselves.

\subsubsection{Random Errors}

In practice, a phased-array implementation cannot provide the exact phase and gain response that are desired for the beamforming pattern. There are mismatches be- 


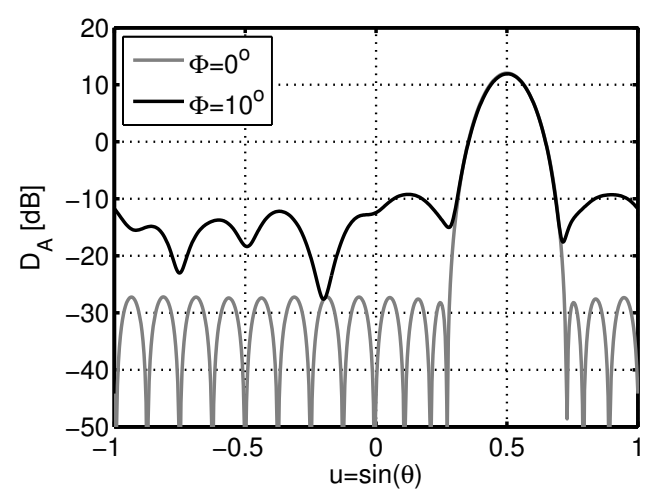

(a)

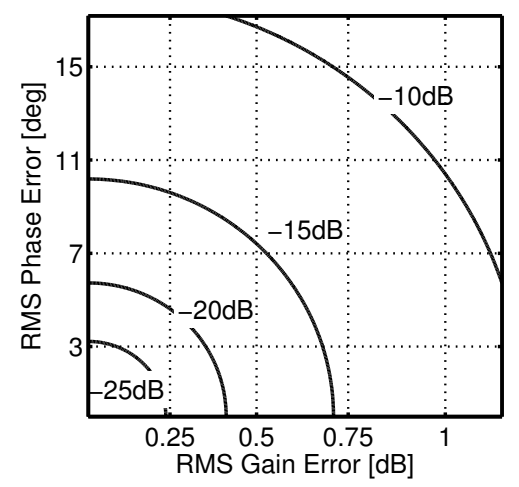

(b)

Figure 2.10: (a) Directivity with and without random phase errors and (b) graph for determining the mean sidelobe level due to random phase and gain errors.

tween components during the technology processing and mechanical variations in the assembly of the dies and PCB. Accumulating all effects for each antenna element into phase error $\Phi_{n}$ and gain error $\delta_{n}$, the array factor can be expressed as:

$$
A F(u)=\sum_{n=0}^{N-1}\left|a_{n}\right|\left(1+\delta_{n}\right) \cdot e^{j \pi \cdot n \frac{f}{f_{0}}\left(u-u_{0}\right)} e^{j \Phi_{n}} .
$$

Skolnik shows that when these errors are described as additive random errors with a Gaussian distribution and zero mean, the average power array factor is [37]:

$$
|\overline{A F(u)}|^{2}=e^{-\bar{\Phi}^{2}} \cdot\left|A F_{0}(u)\right|^{2}+\left(1-e^{-\bar{\Phi}^{2}}+\bar{\delta}^{2}\right) \cdot \sum_{n=0}^{N-1}\left|a_{n}\right|^{2}
$$

where $\bar{\delta}^{2}$ is the gain ratio variance normalized to unity and $\bar{\Phi}^{2}$ is the phase error variance in radians squared. This result shows that the error-less array factor $\left|A F_{0}(u)\right|^{2}$ is reduced slightly by the $e^{-\bar{\Phi}^{2}}$ factor, but more importantly, an additional term is introduced due solely to the random errors. In fact, the gain and phase errors take a fraction of the energy from the main beam and distribute this energy to the sidelobes. An important observation can be made if equation (2.26) is applied to determine the array directivity:

$$
\left|\overline{D_{A}(u)}\right|^{2}=e^{-\bar{\Phi}^{2}} \cdot\left|D_{A, 0}(u)\right|^{2}+\left(\bar{\Phi}^{2}+\bar{\delta}^{2}\right) .
$$

The error sidelobe level in the directivity plot is independent of the applied weights and solely depends on the added variance power of the phase and gain errors. From these equations, we can see that the random phase and gain errors are treated equally. 
This stands to reason as it is the combined error distance in the phasor diagram that is relevant for constructive and destructive summing.

As an example, Fig. 2.10a plots the array directivity with and without random phase errors. Indeed, the errors increase the sidelobe level while the main beam is almost unaffected. In order to estimate the average sidelobe level, Fig. 2.10b plots $\left(\bar{\Phi}^{2}+\bar{\delta}^{2}\right)$ on a dB scale. For the plotted $10^{\circ}$ of phase error variance, this graph indicates a $-15 \mathrm{~dB}$ average sidelobe level, which is in correspondence with Fig. 2.10a.

In most cases it is not the average sidelobe level, but the peak sidelobe level relative to the main beam which is of interest. In the directivty plot, the main beam increases with $10 \cdot \log (N)$, so that the relative sidelobe level increases with the number of antenna elements. Moreover, Allen has shown that over-designing the average sidelobe level to be $10 \mathrm{~dB}$ below the desired peak sidelobe level is sufficient to ensure good operation in practical cases [38]. This gives the following expression for the sidelobe level $S L$ relative to the main beam.

$$
S L<10 \cdot \log \left(\bar{\Phi}^{2}+\bar{\delta}^{2}\right)-10 \cdot \log (N)+10 \quad[\mathrm{~dB}]
$$

For example, a 4-element array with $2^{\circ}$ root-mean-square (RMS) phase error and 0.2 $\mathrm{dB}$ RMS gain error will have its error sidelobes more than $20 \mathrm{~dB}$ below the main beam. In the case of few antenna elements and adaptive pattern nulling, the same design equations can be used to determine the null depths that can be guaranteed.

\subsubsection{Phase Quantization Errors}

For half $\lambda$ antenna spacing and practical tapers, the beam width can be approximated as $(2.10)$ :

$$
u_{-3 d B} \approx \frac{2}{N} .
$$

In u-space a complete sweep of the beam goes in steps of $u_{-3 d B}$ from $u=-1$ to $u=1$, so that $N$ beams can cover the entire sweep span, with a $3 \mathrm{~dB}$ ripple on reception (Fig. 2.11a):

$$
u_{0}=-1+\frac{2 i}{N}, \quad i=0,1,2, . ., N-1
$$

From the phase shifter weights in narrowband arrays (2.14) we find that the smallest phase steps are for the beam which is pointed one beam width away from broadside $(i=N \pm 1)$. If the phase steps are not small enough, they have to be rounded off and groups of elements with the same phase shift occur, resulting in grating lobes (Fig. $2.11 b)$. So, for a $-3 d B$ scan range in an $N$-element array without grating lobes, the minimum of uniformly quantized phase shifter steps is $N$.

If the required phase shifter steps are not available, quantization lobes will occur in the array factor. Miller has considered the quantization lobe level for a continuous 

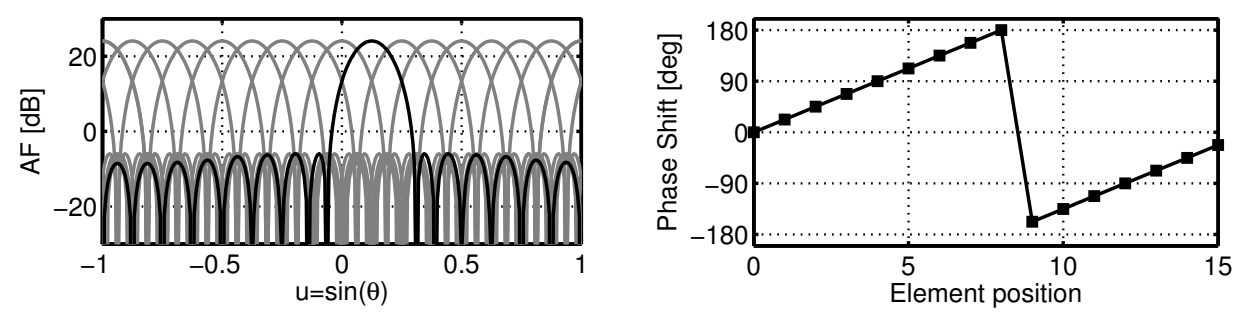

(a)
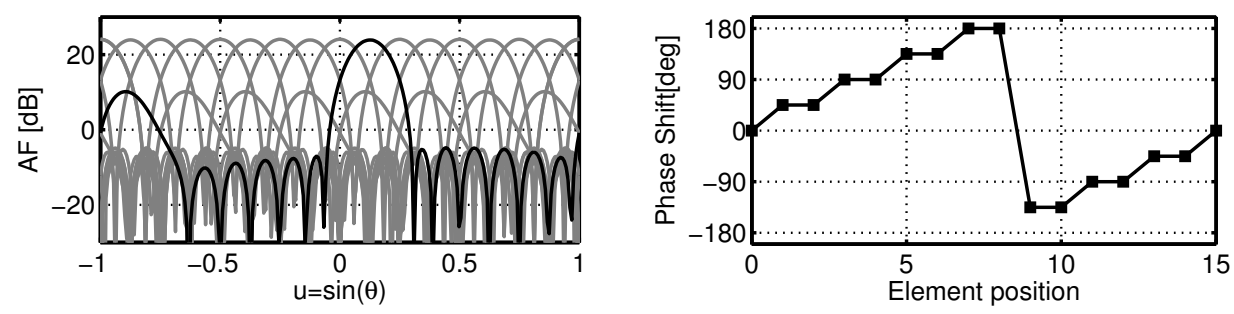

(b)

Figure 2.11: Swept beams in a 16-element array with -30 dB Taylor taper and (a) 4-bit and (b) 3-bit phase quantization.

aperture by assuming the quantization error to have a triangular error distribution [39]. He concluded that for each bit in the phase shifter, the quantization lobes are about $6 \mathrm{~dB}$ below the main beam, a familiar result from amplitude quantization in $\mathrm{AD}$ converters. For a discrete aperture (like a phased array) however, the math is more intricate as the error can become periodic and is no longer randomly distributed. Mailloux [40] has shown that these effects worsen the quantization lobe level to about $4 \mathrm{~dB}$ with respect to a continuous aperture. Therefore, a safe design choice can be made if the following rule of thumb is used:

$$
Q L<-6 b+4 \quad[\mathrm{~dB}]
$$

For example, a 5 bit phase control will result in quantization lobes more than $26 \mathrm{~dB}$ below the main beam.

\subsubsection{Amplitude Quantization Errors}

The effects of amplitude quantization are harder to capture in closed form equations than the effects of phase quantization. The work of Mailloux [40] describes the effect of continuous amplitudes being applied to quantized apertures (in subarrays), but there is little work on closed-form expressions of amplitude quantization. In practice, simulations have to be performed to calculate its effects. 


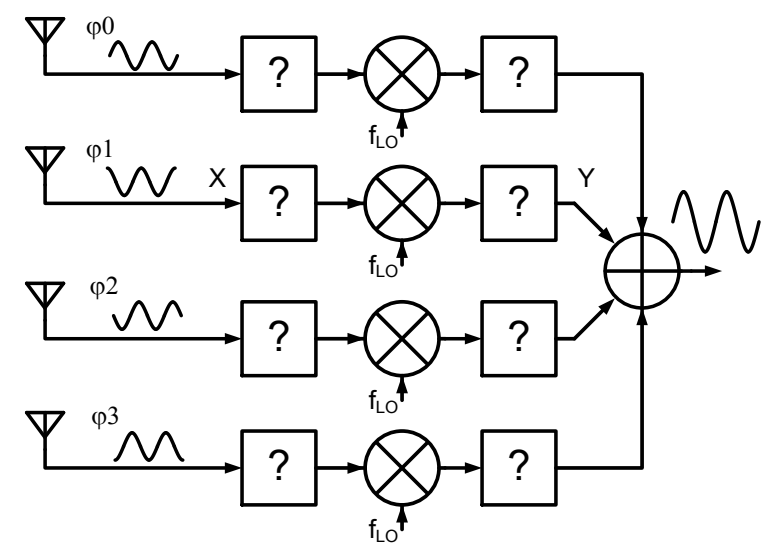

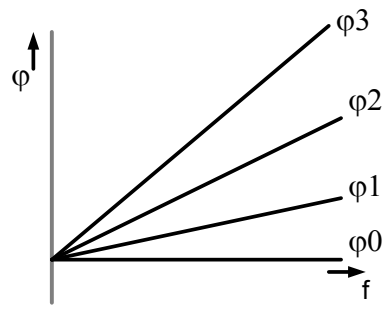

(b)

(a)

Figure 2.12: (a) Beamforming system with downconversion and (b) phases of the incoming signals as a function of frequency.

\subsection{Frequency Translation and Beamforming}

Downconversion is necessary in receivers to convert high frequency radio signals to low frequency baseband signals, suitable for digitizing by an ADC. The inclusion of frequency translation into the beamforming network gives additional degrees of freedom, that might be exploited in the architecture. As Fig. 2.12a indicates, phase shift and/or time delay can be inserted before or after the mixer that performs downconversion.

In particular, this section deals with the different possible cascades that can be constructed from the three building blocks presented in Fig. 2.13, while maintaining phased-array functionality. For each block, a phase plot of the input signal $X$ and output signal $Y$ as function of the frequency $f$ is given to illustrate its operation. It is assumed that all signals have unity magnitude. The phase diagram of the mixer (Fig. 2.13a) visualizes the horizontal shift by frequency $f_{L O}$ that the signal experiences when going from input to output. In contrast, a phase shift (Fig. 2.13b) performs a vertical shift by phase $\Delta \varphi$. The time delay block (Fig. 2.13c) adds phase $2 \pi f \Delta \tau$, changing the slope of the signal phase.

\subsubsection{Effects of Phase Shift and Time Delay}

Concentrating again on Fig. 2.12a, our efforts can be focused on a single path. The antenna signal on the n-th element has delay $\tau_{n}$ :

$$
X=e^{j 2 \pi f\left(t+\tau_{n}\right)} .
$$




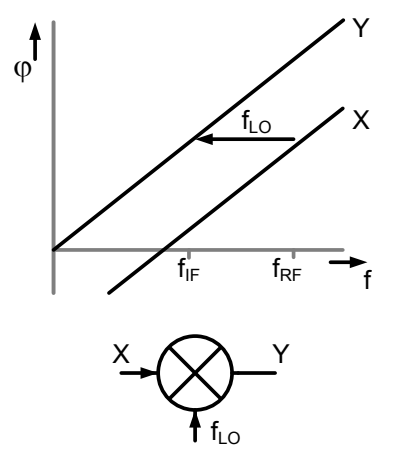

(a)
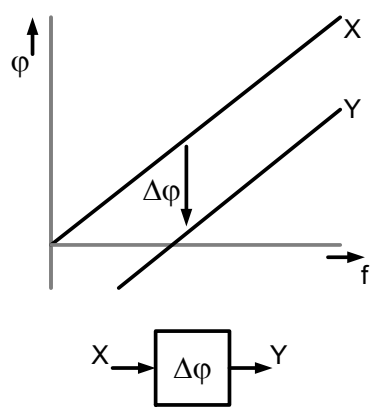

(b)
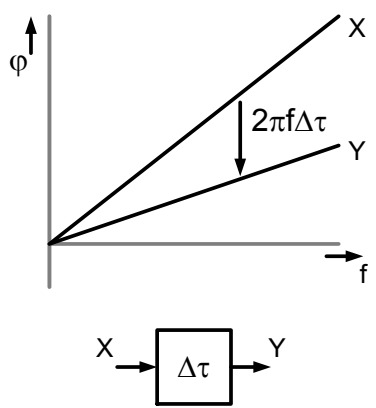

(c)

Figure 2.13: Block diagram and phase plot for (a) a mixer block, (b) a phase shift block and (c) a time delay block.

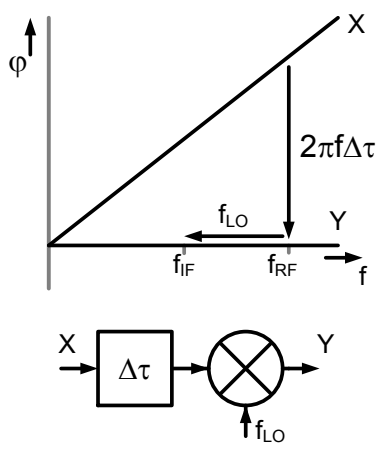

(a)
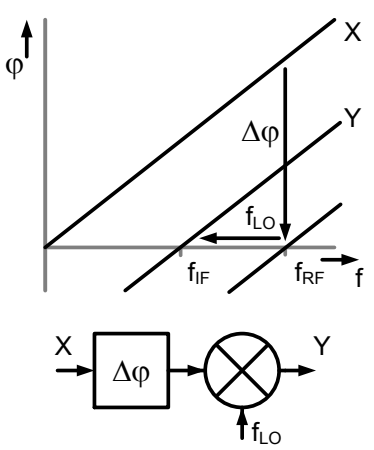

(b)

Figure 2.14: Block diagram and phase plot for (a) RF time delay and (b) RF phase shift.

This delay has to be removed in order to get maximum constructive summing, while downconversion is also performed. Therefore, the ideal signal at node $Y$ can be expressed as:

$$
Y=Y_{0}=e^{j 2 \pi\left(f-f_{L O}\right) t}
$$

The most straightforward solution is presented in Fig. 2.14a. The phase at node $X$ is sloped due to the delay $\tau_{n}$. For constructive summing for all frequencies, the phase should be zero, which is accomplished by first putting a delay block with delay $\Delta \tau=-\tau_{n}$. The phase is now aligned with the frequency axis and a frequency 


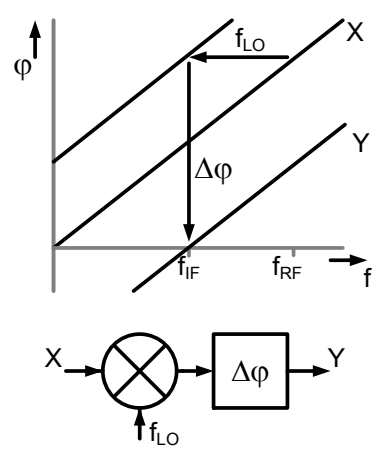

(a)

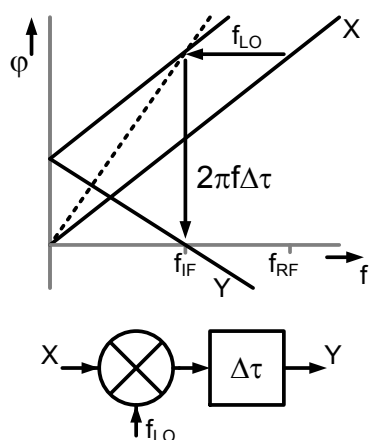

(b)
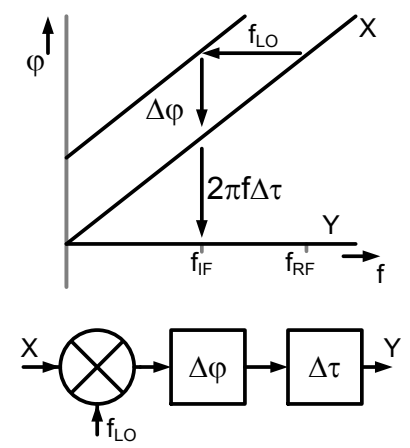

(c)

Figure 2.15: Block diagram and phase plot for (a) IF phase shift, (b) IF time delay and (c) IF time delay + phase shift.

translation can be performed without disrupting the phase:

$$
\begin{aligned}
Y & =e^{j 2 \pi f\left(t+\tau_{n}\right)} \cdot e^{j 2 \pi f \Delta \tau} \cdot e^{-j 2 \pi f_{L O} t} \\
& =e^{j 2 \pi\left(f-f_{L O}\right) t} \cdot e^{j 2 \pi f\left(\tau_{n}+\Delta \tau\right)}=Y_{0} \quad, \Delta \tau=-\tau_{n} .
\end{aligned}
$$

In section 2.1.2, the use of phase shifters to approximate delay in a narrow band was explored, resulting in beam squint. When the delay is replaced with a phase shift (Fig. 2.14b), the output is:

$$
\begin{aligned}
Y & =e^{j 2 \pi f\left(t+\tau_{n}\right)} \cdot e^{j 2 \pi \Delta \varphi} \cdot e^{-j 2 \pi f_{L O} t} \\
& =e^{j 2 \pi\left(f-f_{L O}\right) t} \cdot e^{j 2 \pi\left(f \tau_{n}+\Delta \varphi\right)}
\end{aligned}
$$

The phase $\varphi$ can only be made zero for a single center frequency $\Delta \varphi=2 \pi f_{0} \tau_{n}$ and excess phase remains:

$$
Y=e^{j 2 \pi\left(f-f_{L O}\right) t} \cdot e^{j 2 \pi\left(f-f_{0}\right) \tau_{n}}=Y_{0} \cdot e^{j 2 \pi\left(f-f_{0}\right) \tau_{n}}
$$

By realizing that $\tau_{n}=n \frac{u_{0}}{2 f_{0}}$ and $f-f_{0}=-\Delta f$, it is seen that the excess phase is equal to the previous analysis for phase shifter based arrays in equation (2.16). The increasing phase deviation around the center frequency gives rise to the beam squint. Moving the phase from the RF to the IF port of the mixer (Fig. 2.15a) results in the same output $Y$, as the order of frequency translation and phase shift can be exchanged. It can be stated that a mixer is transparent for phase.

However, if the time delay is moved from RF to IF (Fig. 2.15b), the phase plot reveals that beam squint will appear. Where does the squint come from? Well, a delay at RF frequencies gives a phase line through the origin of the phase plot, but 


\begin{tabular}{|c||c|c|c|c|c|}
\hline Type & RF & IF & $\Delta \varphi$ & $\Delta \tau$ & $\alpha$ \\
\hline \hline TD @ RF & TD & & & $-\tau_{n}$ & 0 \\
\hline PS @ RF & PS & & $2 \pi f_{0} \tau_{n}$ & & 1 \\
\hline TD @ IF & & TD & & $-\frac{f_{0}}{f_{0}-f_{L O}} \tau_{n}$ & $\frac{f_{L O}}{f_{0}-f_{L O}}$ \\
\hline PS @ IF & & PS & $2 \pi f_{0} \tau_{n}$ & & 1 \\
\hline PS+TD @ IF & & PS+TD & $2 \pi f_{L O} \tau_{n}$ & $-\tau_{n}$ & 0 \\
\hline
\end{tabular}

Table 2.1: Beam squint summary.

the downconversion shifts it off the origin:

$$
\begin{aligned}
Y & =e^{j 2 \pi f\left(t+\tau_{n}\right)} \cdot e^{-j 2 \pi f_{L O} t} \cdot e^{j 2 \pi\left(f-f_{L O}\right) \Delta \tau} \\
& =e^{j 2 \pi\left(f-f_{L O}\right) t} \cdot e^{j 2 \pi\left(f \tau_{n}+f \Delta \tau-f_{L O} \Delta \tau\right)}
\end{aligned}
$$

Like the phase shift solution, the phase line can only go through zero for one frequency $f=f_{0}$ :

$$
Y=Y_{0} \cdot e^{j 2 \pi\left(f-f_{0}\right) \frac{f_{L O}}{f_{0}-f_{L O}} \tau_{n}} \quad, \Delta \tau=\frac{f_{0}}{f_{0}-f_{L O}} \tau_{n}
$$

and beam squint occurs for other frequencies. Therefore, it can be stated that a mixer is not transparent for delay. Compared to (2.16), the excess phase has an additional factor of $\frac{f_{0}}{f_{0}-f_{L O}}$, so a time delay at IF will have a larger beam squint than a phase shifter based array.

In order to eliminate the beam squint for IF time delays, an additional phase shift is needed (Fig. 2.15c) to correct for the shift of the phase plot due to downconversion. The horizontal shift in the plot by the downconversion is canceled by the vertical shift of phase shifter, realigning the phase line with the origin. In mathematical form:

$$
\begin{aligned}
Y & =e^{j 2 \pi f\left(t+\tau_{n}\right)} \cdot e^{-j 2 \pi f_{L O} t} \cdot e^{-j \Delta \varphi} \cdot e^{j 2 \pi\left(f-f_{L O}\right) \Delta \tau} \\
& =Y_{0} \quad, \Delta \tau=-\tau_{n}, \quad \Delta \varphi=2 \pi\left(f-f_{L O}\right) \tau_{n} .
\end{aligned}
$$

To summarize the beam squinting results for the different architectures, equation (2.18) is modified with an additional $\alpha$ factor.

$$
\frac{\Delta u(f)}{u_{0}}=\alpha \frac{\Delta f}{f}
$$

Or in other words, the $\alpha$ parameter links the relative frequency deviation to the relative beam shift. The results thus obtained are summarized in Table 2.1.

\subsubsection{Transformations}

In the analysis so far, only the RF and IF possibilities for phase shift and time delay were considered. If the $\mathrm{LO}$ port of the mixer is also taken into account as an option to 


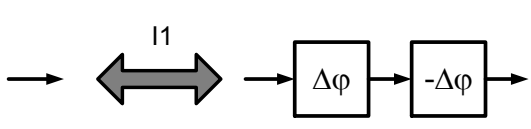

(a)

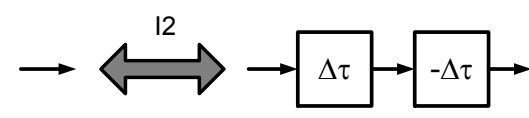

(b)

Figure 2.16: Identities (a) I1 and (b) I2.

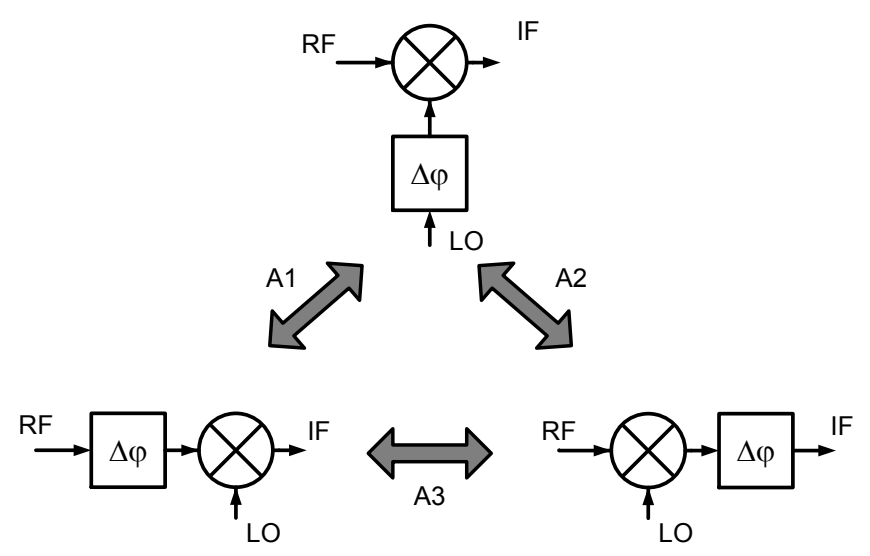

Figure 2.17: Transformation A1, A2 and A3.

insert a phase shift or time delay, the number of combinations increases many times. A more practical approach is to find configurations that are equivalent, so that an unfamiliar form can be transformed into a familiar one.

First, the two identities in Fig. 2.16 are considered. Identity I1 states that a phase shift followed by the same negative phase shift is equivalent to doing nothing. For delay, the same is stated in identity I2. These identities might look trivial, but they allow the spawning of phase shift and time delay blocks in any point of a block diagram.

From the previous analysis, it was concluded that a mixer is transparent for phase. A phase shift at IF is therefore equivalent to a phase shift at $\mathrm{RF}^{4}$ :

$$
I F(t)=L O(t) \cdot R F(t) \cdot e^{j 2 \pi \Delta \varphi} \equiv \quad I F(t) \cdot e^{-j 2 \pi \Delta \varphi}=L O(t) \cdot R F(t) .
$$

Of course, this property can be extended to the LO port as the mixer is in essence a three-port device. These observations are captured in the three A transformations in figure 2.17, stating that a phase shift can be shifted through the mixer to another port.

\footnotetext{
${ }^{4}$ The sign of the block at the IF port in the block diagram is inverted with respect to the equation, because the arrow points outwards of the mixer.
} 


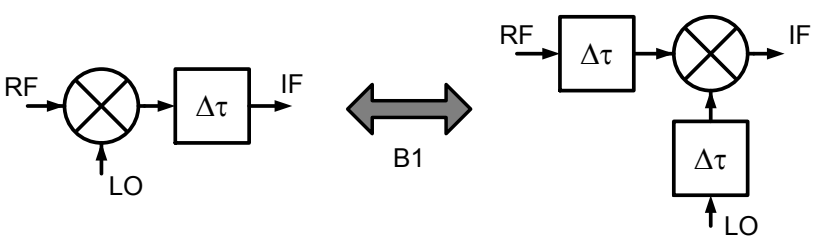

(a)

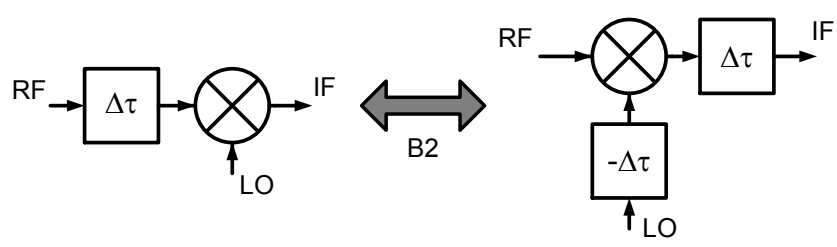

(b)

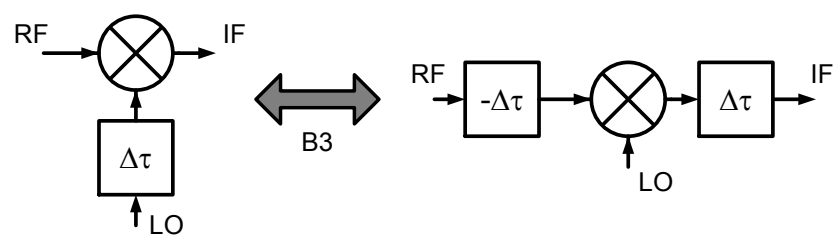

(c)

Figure 2.18: Transformation (a) B1, (b) B2 and (c) B3.

This property is not the same for a time delay. Instead, the principle of causality can be used to derive its appropriate transformations. Consider a system with a time delay at the IF port and a system with a time delay at the RF and LO port. If these system are viewed as a black box, they cannot be distinguished from one another. Therefore the equivalency

$$
I F(t-\Delta \tau)=L O(t) \cdot R F(t) \quad \equiv \quad I F(t)=L O(t+\Delta \tau) \cdot R F(t+\Delta \tau)
$$

exist. This property is captured in the B1 transformation in Fig. 2.18a. To treat a system with a time delay at the RF port, first apply identity 2 to the LO port, then apply transformation B1 to arrive at transformation B2 in Fig. 2.18b. With a similar procedure, transformation B3 in Fig. 2.18c can be derived. This illustrates the power of these transformations, as new block diagrams are created from known ones.

So far, the phase shift and time delay transformation stand on their own. They are connected by applying the restriction that the LO port does not contain a full spectrum signal, but only carries the single-tone clock frequency $f_{L O}$. Now, a time delay at the LO port is equal to a phase shift at the LO port:

$$
\Delta \varphi=2 \pi f_{L O} \Delta \tau
$$



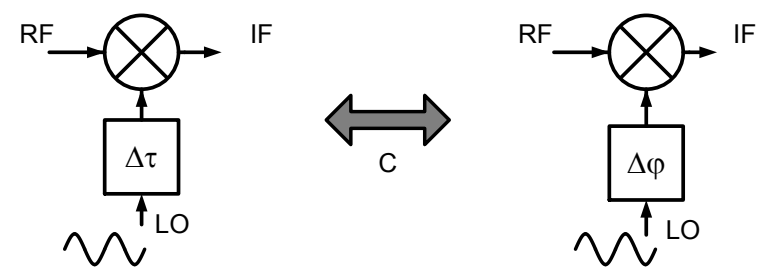

Figure 2.19: Transformation $\mathrm{C}\left(\Delta \varphi=2 \pi f_{L O} \Delta \tau\right)$.
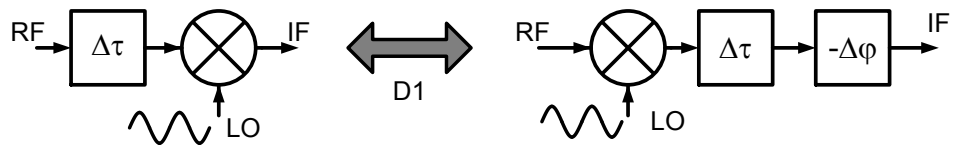

(a)
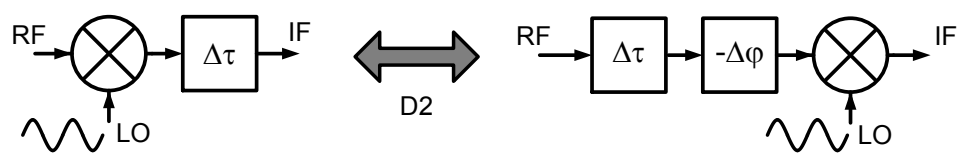

(b)

Figure 2.20: Transformation (a) D1 and (b) D2 $\left(\Delta \varphi=2 \pi f_{L O} \Delta \tau\right)$.

which is stated in the $\mathrm{C}$ transformation in Fig. 2.19.

This opens up new possibilities. Starting with a time delay at RF, one can apply first the B2 transformation to move the delay to the IF and LO port, followed by the $\mathrm{C}$ transformation to turn the LO delay into a phase shift. After moving the phase shift to the IF port with transformation A2, the new D1 transformation is finished (Fig. 2.20a). What was concluded in the previous subsection with some math is now again apparent from these transformations: a time delay at RF is equivalent to a time delay and phase shift at IF.

Starting with a time delay at IF and applying transformations B1, C and A1 yields transformation D2 in Fig. 2.20b. Now, it is seen that a time delay at IF is equivalent to a time delay and phase shift at RF. The extra phase shift will produce beam squint, as was calculated earlier.

\section{$2.3 \quad$ RF System Design Aspects}

Now that the theoretical framework for beamforming has been laid out, it can be applied to receivers for consumer wireless standards. Table 2.2 lists several of these 
Table 2.2: Consumer Wireless Standards

\begin{tabular}{|c|c|c|c|}
\hline Standard & $\begin{array}{c}\text { Frequency } \\
\text { band } \\
{[\mathrm{GHz}]}\end{array}$ & $\begin{array}{c}\text { Channel } \\
\text { bandwidth } \\
{[\mathrm{MHz}]}\end{array}$ & $\begin{array}{c}\text { Smallest } \\
\text { wavelength } \\
{[\mathrm{cm}]}\end{array}$ \\
\hline \hline GPS & 1.57 & 20 & 20 \\
\hline DECT (Europe) & $1.88-1.9$ & 1.7 & 14 \\
\hline UMTS & $1.9-2.1$ & 5 & 14 \\
\hline Bluetooth & $2.4-2.5$ & 1 & 12 \\
\hline $\mathrm{WiFi} 802.11 \mathrm{~b} / \mathrm{g} / \mathrm{n}$ & $2.4-2.5$ & 20 or 40 & 12 \\
\hline $\mathrm{WiFi} 802.11 \mathrm{a} / \mathrm{n}$ & $4.9-5.9$ & 20 or 40 & 5 \\
\hline
\end{tabular}

standards, along with their channel bandwidth and the smallest wavelength associated with their frequency band. From these specifications, several key system design aspects can be derived.

\subsubsection{Bandwidth and Architecture}

Among the standards, the highest fractional bandwidth is still only $1 \%$. So all can be considered narrowband standards and beam squinting becomes a problem only when more than 100 antenna elements are needed. Therefore it is safe to use phase shifters instead of time delays to steer the beam.

The respective carrier wavelengths are in the order of 5 to $20 \mathrm{~cm}$. With halfwavelength spacing between antenna elements and usable form factors in consumer products, only a few antenna elements can be supported. To meet these requirements, a number of 4 elements has a reasonable aperture and still beamforming properties. Tapering is not an option to ensure low sidelobes, so adaptive nulling should be used to cancel the dominant interferer.

In this chapter, it was demonstrated that transformations A1, A2 and A3 (Fig. 2.17) can be used to position the phase shift at either the RF, LO and IF port of a downconverter in the beamforming system. Putting the Phase shifter at the RF port requires it to support the high bandwidth of the entire RF carrier and can add little parasitics to input and output nodes. The LO port location has to deal with the large LO swing. Therefore, phase shifters at the IF port are favorable, as parasitics can be absorbed and the bandwidth has been reduced to the necessary channel bandwidth.

With these considerations, a Zero-IF receiver can be transformed into a beamforming receiver as shown in Fig. 2.21. A split is made right after the downconverter, and phase/amplitude control blocks are added together with a summing node. Only the circuits before the split are duplicated for each antenna element, while the circuits after the split are kept singular. Note that the summing node includes a scaling factor of $1 / N$, which arises naturally when summing in the current domain. 

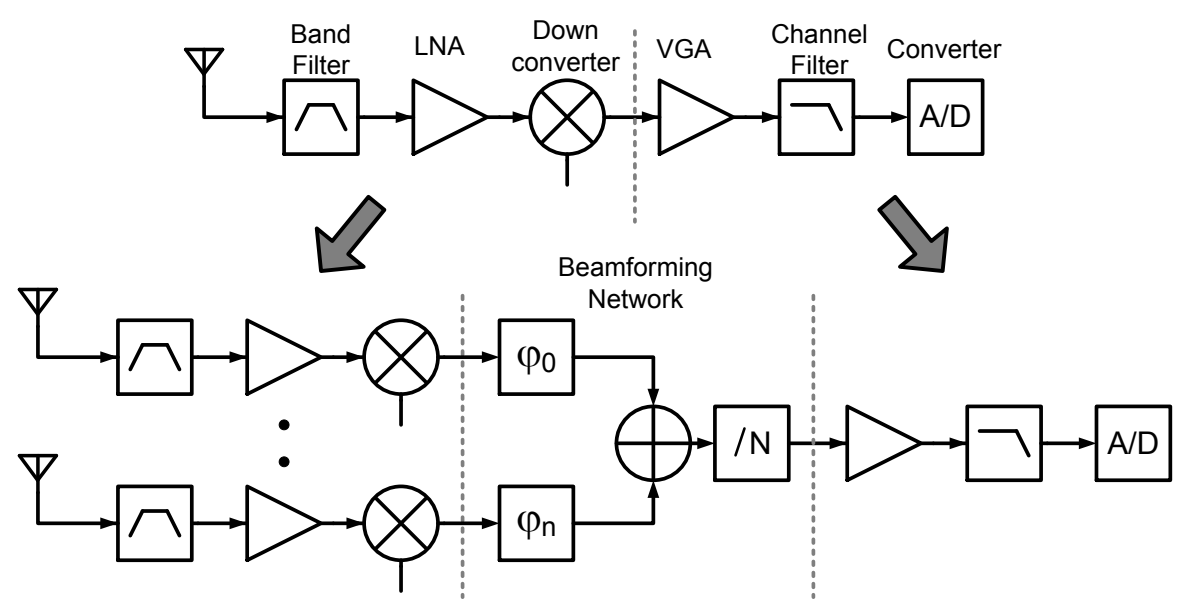

Figure 2.21: Transforming a Zero-IF receiver into a beamforming receiver.

\subsubsection{Linearity and Noise}

In transforming the singular receiver into a beamforming receiver, the overall aim was to improve sensitivity and reduce the distortion due to large-swing interferers in the baseband processing. For a singular receiver, the contribution of a circuit block's noise and distortion to the overall system noise and distortion can be calculated when the gain of the signal to the input of that block is known. These equations only apply for single-input, single-output systems, so some insights are necessary to apply them to the multiple-input, single-output beamforming system. In order to keep the results insightful and simple, it is assumed that the beamforming network introduces no noise or distortion and the phase/amplitude blocks have unity gain.

First, consider the situation for signals in the main beam of the antenna pattern, so that no spatial filtering occurs. This produces signals at the output of the downconverters with the same amplitude as on the splitting node in the singular receiver, which are coherently summed in the summing node. It is important to realize that due to the scaling factor $1 / N$, the signal amplitude at the output of the beamforming network is the same as at each of its inputs. This means that the signal amplitude at the input of each block in the beamforming receiver has not changed with respect to the singular receiver.

From this result, it can immediately be concluded that the in-band, in-beam linearity performance of the beamforming receiver is equal to that of the singular receiver. Of course, the spatial filtering will ensure that the out-of-beam linearity due to the blocks after the summing node is still increased.

In the section on directivity and sensitivity 2.1 .3 , it was concluded that the sensitivity increases with $3 \mathrm{~dB}$ per doubling of array elements, due to the fact that noise 
adds non-coherently and signals add coherently in the summing node. To keep up with the decreasing sensitivity of the circuits blocks before the beamforming network, it can be found that the noise of the circuit blocks after the summing node has to also decrease proportionally. From impedance scaling theory, it is known that noise and supply power scale linearly as long as the impedance level is scaled accordingly, so that a $3 \mathrm{~dB}$ increase of sensitivity corresponds to a doubling in supply power for the circuits after the summing node. But, the doubling of the number of array elements also corresponds to a doubling in supply power of the circuits before the summing node, which can be explained as an alternate way of impedance scaling. So, an important conclusion is reached: The sensitivity improvement from beamforming requires a proportional increase in supply power, as with impedance scaling.

Impedance scaling a complete singular receiver usually does not work, as the antenna impedance is fixed. For a beamforming receiver, it is as if the antennas are in parallel and result in a virtual antenna with lower impedance.

\subsection{Conclusions}

The fundamental properties of linear phased arrays were summarized in the first part of this chapter. The basic beam pattern was introduced and the effects on it by beamsteering, amplitude tapering and the phase shifter approximation was summarized, along with the degradation due to errors in the array.

Next, a framework for including downconversion in the beamforming architecture has been presented, along with transformation methods to move time delay and/or phase shift between the RF, LO and IF domain. It was found that the reallocation of the beamforming to the $\mathrm{LO}$ or RF domain can give rise to beam squinting, as is the case with the phase shifter approximation in general array theory. Furthermore, the linearity and sensitivity properties of beamforming circuit implementations were covered, concluding that beamforming can be viewed as a form of impedance scaling. 


\section{Chapter 3}

\section{Highly-Linear Mixer-First Receiver Front-Ends}

In the previous chapter the transparency of mixers for phase shifter based beamforming was introduced, such that beamforming could be applied at either RF or IF. For applications where IF beamforming is used for interferer rejection, interferers are rejected at the summation point. This means that the major part of the receiver frontend is still subjected to interferes, while the baseband chain is protected. Therefore, the downconversion and first amplification stage must still be able to withstand these large signals. In this chapter we explore the possibilities for a highly-linear receiver front-end in advanced CMOS.

The key radio receiver specification in this case is the spurious free dynamic range (SFDR), characterizing the maximum distance from signal to the noise plus distortion. The SFDR is limited by the linearity and the noise floor. If the third order distortion is dominant, the SFDR can be expressed as [41]:

$$
S F D R=\frac{2}{3}[I I P 3-N F+174 \mathrm{dBm} / \mathrm{Hz}-10 \cdot \log (B W)]
$$

where $N F$ is the noise figure, IIP3 is the input-referred third order intercept point and $B W$ is the channel bandwidth. As receivers already have a low noise figure and a channel bandwidth defined by the radio standard, there is mainly room for improving the SFDR by increasing the linearity. It is challenging to maintain or even improve linearity intercept points in future CMOS processes with lower supply voltages, due to the strong relation between distortion and voltage swing.

In a conventional LNA-first receiver (Fig. 3.1, top), the bandpass-filtered antenna input is first amplified by the LNA, so the mixer noise will have a small contribution to the system noise figure. The linearity of the LNA is limited due to the low supply 

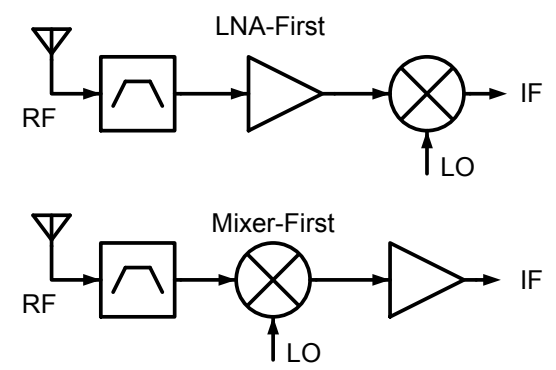

Figure 3.1: LNA-first (top) and mixer-first (bottom) receiver architecture.

voltage and limited loop gain available at RF to linearize with feedback. Moreover, after the LNA gain, the mixer linearity becomes even tougher.

If amplification is postponed to IF, much more loop gain is available to linearize the amplifier. This leads to the mixer-first receiver concept (Fig. 3.1 bottom). The linearity of the mixer is relaxed and can be maximized by using passive mixers. Problem now is maintaining a good system noise figure, despite of the mixer conversion loss and its inherently high noise figure.

\subsection{Switched-RC Mixers and Samplers}

Mixing and sampling seem to be quite different functions and are analyzed differently, but can be implemented by similar circuits. Some examples are shown in Fig. 3.2.

The basic building block of these circuits consists of a resistor and a capacitor in series, which are switched to the input voltage $V_{i n}$. As there is no parallel resistance across the capacitance when the switch is off, the capacitor holds its charge in the off-state. The circuits are purely passive, because the switches act as time-variant resistances and no power gain is possible.

Individual switched-series-RC circuits have been analyzed on a case-by-case basis in literature by posing restrictions on the design parameters. The differential switching mixer (Fig. 3.2b) has a fixed 50\% duty cycle [42]. The switched-RC of Fig. 3.2c is in use as a sampling mixer [43][44], but also as a track-and-hold sampler [45]. In both cases the RC-time is considered to be infinitely small in order to simplify the analysis. The conversion gain and noise figure for the I/Q image reject mixer (Fig. $3.2 \mathrm{~d}$ ) as proposed by Tayloe [46] have only been calculated for DC output frequency [47].

In this section a unified frequency domain analysis is proposed, that can be applied to all of these switched-series-RC circuits, while including arbitrary duty cycle, RCtime and output frequency. 


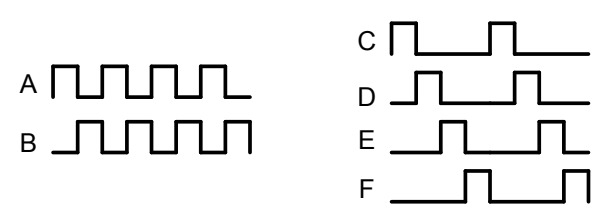

(a)

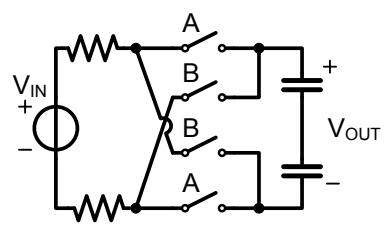

(b)

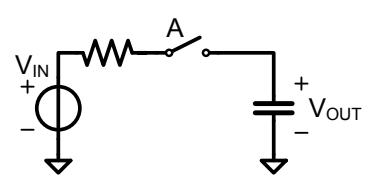

(c)

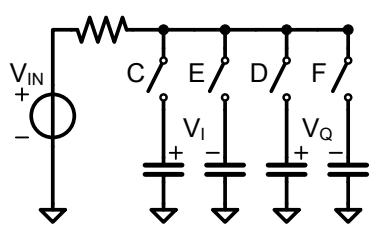

(d)

Figure 3.2: (a) Polyphase clocks A-F for switched RC application examples: (b) Switching Mixer (c) Sampling Mixer / Track and Hold (d) I/Q Mixer.

\subsubsection{LPTV Systems}

The switching nature of the kernels requires the use of linear periodically time variant (LPTV), instead of linear time invariant (LTI), network theory. The time-invariance in an LTI network dictates that a sinusoidal signal with frequency $f_{i}$ at the input of the network results in a sinusoidal tone at its output with the same frequency, albeit with a change in amplitude and phase. The amplitude and phase characteristic over frequency is defined as the transfer function.

In contrast, the impulse response of an LPTV network is time-variant and repeats itself after the time period $T_{s}$. Fig. 3.3 shows a generic model for such a system. There are several paths from input to output, each consisting of a frequency shift and LTI filter $H_{n}$. An input spectrum can thus be translated to multiple locations in the output spectrum, with frequency shifts $n \cdot f_{s}$, where $n$ is an integer and $f_{s}$, the repetition frequency, is the inverse of $T_{s}$.

This statement can be captured into the equation [48]:

$$
V_{o}\left(f_{o}\right)=\sum_{n=-\infty}^{\infty} H_{n}\left(f_{o}\right) V_{i}\left(f_{o}-n f_{s}\right)
$$

$H_{n}\left(f_{o}\right)$ constitute the harmonic transfer functions (HTFs) that define the response of the LPTV system. Due to the frequency shifts, the frequency variable $f_{i}$ in the input spectrum $V_{i}$ has to be separately defined from the frequency variable $f_{o}$ in the output spectrum $V_{o}$. For each frequency shift, with harmonic index $n$, the relation between input and output frequency is defined as:

$$
f_{i}=f_{o}-n f_{s}
$$




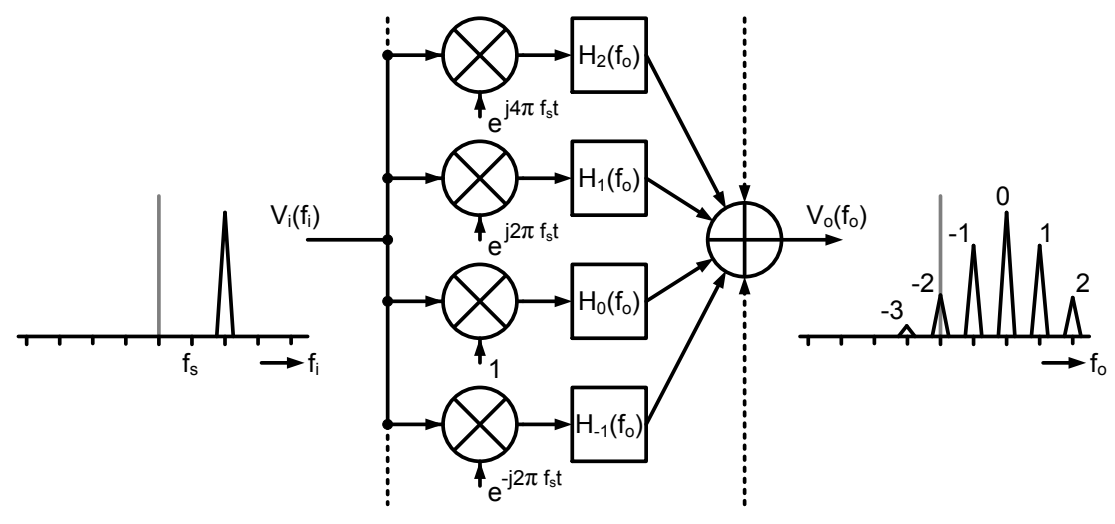

Figure 3.3: Generic frequency domain model of an LPTV system with harmonic transfer function $H_{n}$.

The frequency shifting property of LPTV systems makes them inherently suitable for implementation as mixers. One example is given in Fig. 3.4a, where a multiplication is performed of the input signal with a rectangular clock signal. The multiplication in the time domain is equivalent to a convolution in the frequency domain with the Fourier transform of the clock wave.

For a periodic clock, this transform consists of a train of impulses weighted with the sinc function [49]. The sinc definition used here is the normalized sinc function as defined in (B.1), which is commonly used in digital signal processing and information theory. The convolution of the input signal with an impulse train can be understood to be the frequency shifting property of LPTV systems and some reshuffling gives the form needed for (3.2). The resulting HTFs are the Fourier coefficients of the clock wave:

$$
H_{n, \text { mixer }}=\frac{1-e^{-j \pi n}}{j 2 \pi n}=\operatorname{sinc}(n / 2) \cdot e^{-j \pi n / 2} .
$$

In a mixer design, one of the frequency shifts, with index $n=w$, is the desired conversion. For frequency downconversion, this is usually $n=-1$. Other shifts needs to be suppressed, either by filtering or multi-path polyphase techniques (section 3.1.4).

Switching circuits are also used as sampler in discrete-time systems. Fig. 3.4b gives an example of a sample-and-hold system. The sample process is represented by multiplying the input signal with an impulse train in the time domain, after which a zero-order-hold $(\mathrm{ZOH})$ holds the sample till the next is available. As with the mixer, the multiplication with an impulse train gives rise to the frequency shifting property of LPTV systems. For the sampler however, the ZOH perform a convolution of the sampled input signal with a rectangular pulse in the time domain. This is equivalent to a multiplication in the frequency domain with the Fourier transform of a rectangular 


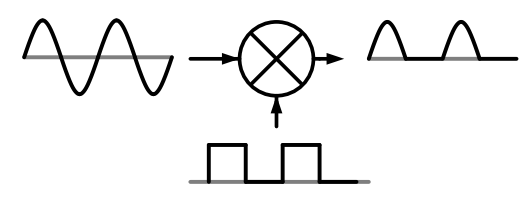

(a)

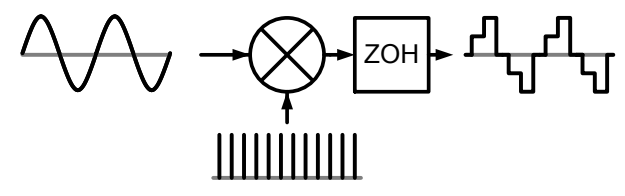

(b)

Figure 3.4: Time domain example of (a) switching mixer and (b) sample and hold.

pulse [49], and the HTFs can be written down as:

$$
H_{n, \text { sampler }}=\frac{1-e^{-j 2 \pi f_{o} / f_{s}}}{j 2 \pi f_{o} / f_{s}}=\operatorname{sinc}\left(f_{o} / f_{s}\right) \cdot e^{-j \pi f_{o} / f_{s}} .
$$

Comparing equations (3.4) and (3.5), it is seen that mixing with a rectangular clock results in HTFs weighted by the sinc as function of harmonic index $n$, while performing a sample-and-hold results in HTFs weighted by the sinc as function of output frequency $f_{o}$.

Note that the mixing properties of a system are defined in the frequency domain and linked to the frequency shifting properties of LPTV systems. On the other hand, sample-and-holding is defined in the time domain, indicating if the output has voltage steps corresponding to input samples. It is therefore possible for a circuit to have both qualities, explaining why the exact same circuit in Fig. 3.2c is used both as mixer and sampler.

\subsubsection{Decomposition into Polyphase Kernels}

Returning to the circuits in Fig. 3.2, we would like to determine the HTFs for each of them. The complexity of the LPTV calculation grows by the number of circuit states, which in this case are the capacitor voltages in the circuit, and the calculation has to be repeated for each circuit. One important observation about these circuits can be made though: the clock phases are polyphase, defined as that they have the same duty cycle and start at uniformly spaced intervals within the period time, avoiding clock overlap.

The absence of clock overlap results in the capacitor voltage being independent of each other, so that they can be evaluated separately. Moreover, the equal duty cycles and uniform distribution of the clock phases ensures that the calculation of each capacitor voltage is essentially the same, except for a shift in time. Keeping these properties in mind, the determination of the circuit's response can now be simplified. The analysis is broken up into two separately evaluated parts: 


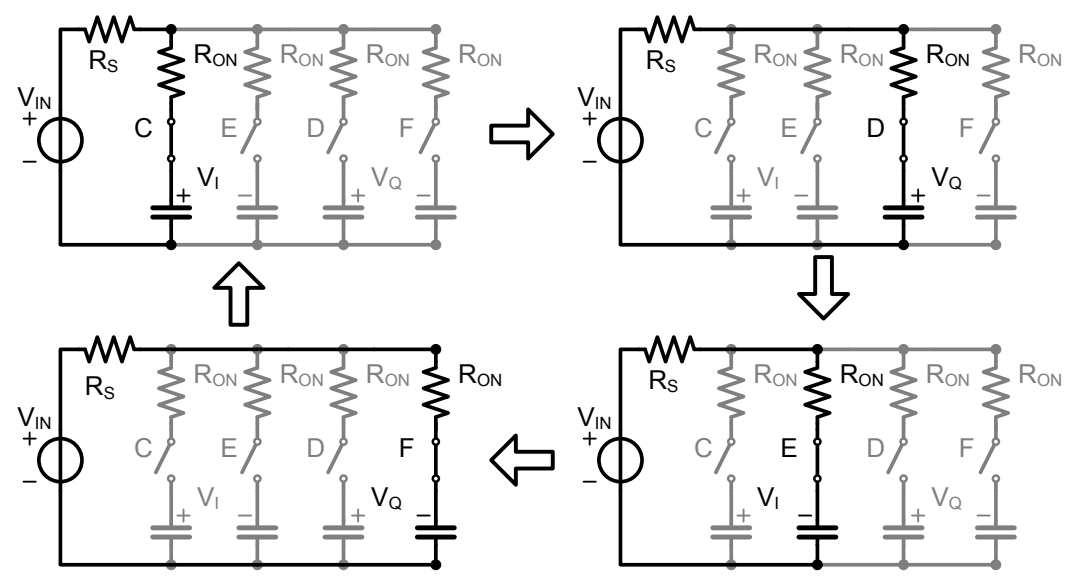

Figure 3.5: Polyphase SE kernel example.
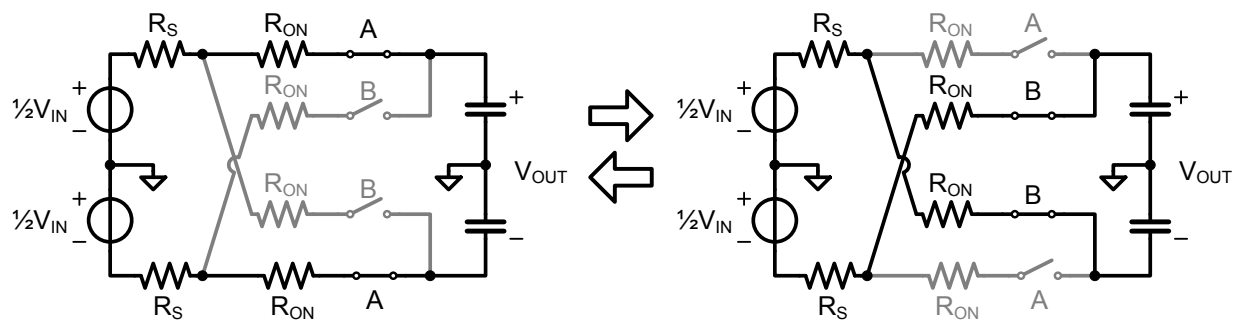

Figure 3.6: Polyphase DI kernel example.

1. Calculate the transfer to a single capacitor voltage with a single-state LPTV calculation.

2. Calculate the effect of combining the capacitor voltages in a polyphase system.

With this approach, the combination of basic single-state building blocks (kernels) into complex multi-state systems is possible, severely simplifying the analysis.

To clarify the polyphase kernel concept, the I/Q mixer of Fig. 3.2d is plotted step-by-step in Fig. 3.5. The switches are modeled with infinite off-resistance and finite on-resistance $R_{o n}$. The source is characterized by the resistance $R_{s}$. During each of the switch intervals, there is current flowing in a single loop from the source through $R_{s}$ and $R_{o n}$ into the capacitor, and back to the source. For calculating the transfer function, $R_{s}$ and $R_{o n}$ can be replaced by the equivalent series resistance:

$$
R=R_{s}+R_{\text {on }}
$$

Analysis of this current loop, defined as the single-ended (SE) kernel (Fig. 3.7b), is sufficient to find the capacitor voltage. 


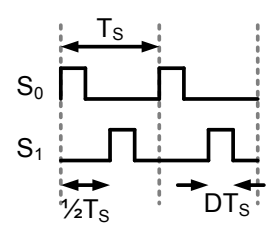

(a)

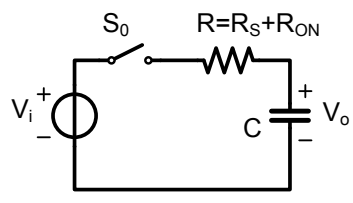

(b)

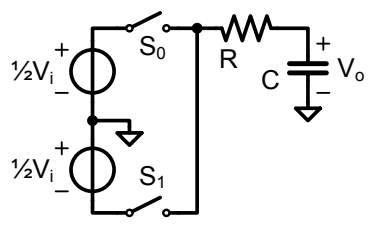

(c)

Figure 3.7: (a) Switching clocks $S_{0}$ and $S_{1}$ with duty cycle $D$ for the (a) Single-ended (SE) and (b) Differential (DI) kernel.

For differential inputs a second kernel is needed, as the example in Fig. 3.6 shows. Here the same capacitor is connected to the input twice in each period. In the definition of the differential (DI) kernel (Fig. 3.7c), the second switch is delayed half a period after the first switch and has the same duty cycle.

\subsubsection{Kernel Analysis}

For calculating the HTFs of the SE and DI kernel, the method based on state space ${ }^{1}$ system modeling as described by Ström and Signell [51], which is described in detail in appendix A.1, is used. In summary, this method uses the fact that LPTV circuits with ideal switches have a finite number of intervals (the clock phases), during each of which the circuit follows LTI behavior. By connecting the initial and final values of these intervals, the circuit states sampled at the switching moments can be determined. Using the switching moments as initial conditions, the response during the intervals can now be inserted to complete the total circuit response.

Only two degrees of freedom are present in the kernels: the duty cycle $D$ and the RC-time associated with the resistor and capacitor. The duty cycle $D$ is defined as the fraction of the period $T_{s}$ that the switch is closed. The RC bandwidth is defined as:

$$
f_{r c} \equiv \frac{1}{2 \pi R C} .
$$

Using the state space system modelling, Appendix A.2 derives the exact SE kernel HTFs for all duty cycles $D$ and $R C$ bandwidths $f_{r c}$ :

$$
H_{n, S E}\left(f_{o}\right)=P_{S E}\left(f_{o}\right)\left[\frac{1-e^{-j 2 \pi D n}}{j 2 \pi n}+\frac{e^{j 2 \pi(1-D) f_{o} / f_{s}}-1}{j 2 \pi f_{o} / f_{s}} G_{S E}\left(f_{o}-n f_{s}\right)\right],
$$

where $P_{S E}$ is a filter as function of the output frequency and $G_{S E}$ is a filter as function of the input frequency.

The HTFs can also be represented by the block diagram in Fig. 3.8a. There are two parallel paths, the upper corresponds to the interval in which the switch is closed

\footnotetext{
${ }^{1}$ For a treatment on state space system theory, consult [50].
} 


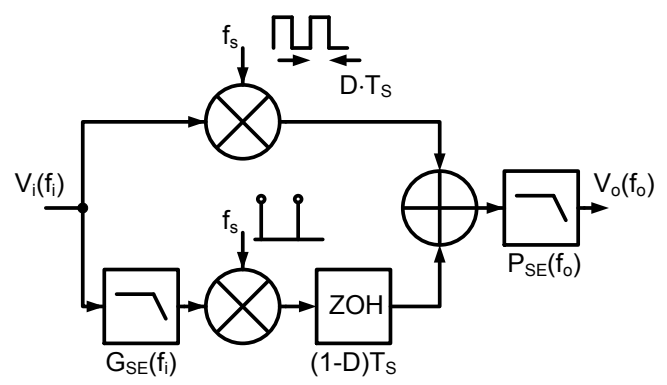

(a)

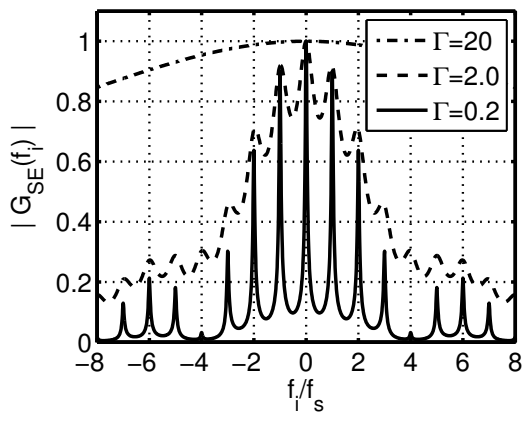

(b)

Figure 3.8: (a) SE kernel block diagram, equivalent to equation (3.8). (b) Filter $G_{S E}$ plotted for several $\Gamma$ and duty cycle $D=0.25$.

and the input is tracked. This tracking interval reflects in a mixing operation in the block diagram, with duty cycle $D$. The lower path corresponds to the interval when the switch is opened and there is a voltage being held on the capacitor. This holding interval is reflecting in the sampler and zero-order-hold in the diagram.

The bandwidth of the output node, after summation of the contributions of the two intervals, is limited by the single-pole lowpass output filter $P_{S E}$ :

$$
P_{S E}\left(f_{o}\right)=\frac{1}{1+j \frac{f_{o}}{f_{r c}}}
$$

Input filter $G_{S E}$ has a more complex expression:

$$
G_{S E}\left(f_{i}\right)=\frac{e^{j 2 \pi D f_{i} / f_{s}}-e^{-2 \pi D f_{r c} / f_{s}}}{e^{j 2 \pi f_{i} / f_{s}}-e^{-2 \pi D f_{r c} / f_{s}}} \frac{1}{1+j \frac{f_{i}}{f_{r c}}} .
$$

The factor $2 \pi D \frac{f_{r c}}{f_{s}}$ can be written as the ratio between switch-on time and the RCtime constant, it will be designated with $\Gamma$ :

$$
2 \pi D \frac{f_{r c}}{f_{s}}=\frac{D T_{s}}{R C} \equiv \Gamma .
$$

A plot of the $G_{S E}\left(f_{i}\right)$ is given in Fig. $3.8 \mathrm{~b}$ for three values of $\Gamma$ as defined in equation (3.11). The filter represents the transfer function at the switching-moments and determines the filtering of the samples for the $\mathrm{ZOH}$. From the plots, it is evident that the filter is differently shaped for different values of $\Gamma$.

In fact, simpler approximations for the HTFs can be calculated in the limit for $\Gamma \rightarrow 0$ and $\Gamma \rightarrow \infty$. The design space can be divided into two regions, with border $\Gamma=2$. The choice for this particular border (as opposed to $\Gamma=1$ or any other value) will be motivated at the end of this section. 

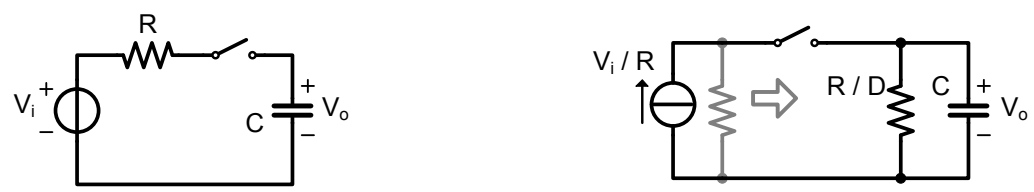

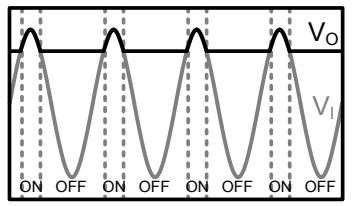

(a)

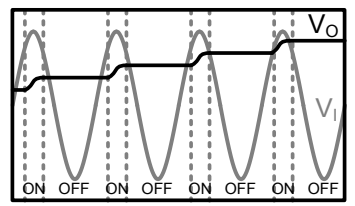

(b)

Figure 3.9: SE Kernel (a) Thevenin equivalent for $\Gamma \gg 2$ (sampling region) and (b) Norton equivalent for $\Gamma \ll 2$ (mixing region).

When the RC-time constant is relatively small $(\Gamma \gg 2)$, the behavior of the circuit can be understood by looking at the node voltages (in the Thevenin equivalent in Fig. $3.9 \mathrm{a})$. When the switch is closed the capacitor voltage will follow the input, and when the switch is open the last voltage will be held on the capacitor. Such operation is commonly referred to as track-and-hold and is widely used in samplers. Therefore the part of the design space for which $\Gamma \gg 2$ is defined as the sampling region.

Appendix A.3 calculates approximation HTFs for the sampling region $(\Gamma \gg 2)$ :

$$
\begin{aligned}
& H_{n, S E}\left(f_{o}\right) \approx \\
& D \operatorname{sinc}(D n) e^{-j \pi D n}+(1-D) \frac{\operatorname{sinc}\left((1-D) f_{o} / f_{s}\right)}{1+j \frac{-n f_{s}}{f_{r c}}} e^{-j \pi\left[(1-D) f_{o} / f_{s}+2 D n\right]} .
\end{aligned}
$$

The equation reflects the tracking and holding interval in its two terms. In the approximation, output filter $P_{S E}$ is considered to be flat, while single-pole lowpass input filter $G_{S E}$ provides filtering of higher harmonic indices for the sample-and-hold.

For a relatively large time constant $(\Gamma \ll 2)$, the operation of the circuit can be understood by looking at the branch currents (the Norton equivalent in Fig. 3.9b). Due to the large RC-time constant, the capacitor voltage changes only slowly each clock cycle. The resistor can conceptually be moved after the switch ${ }^{2}$, but is sized larger (by division with the duty cycle) as it is connected to the capacitor for the full clock period. We see that the input current is multiplied by the clock and low pass filtered at the output, identical in operation to a switching mixer. Therefore the part of the design space for which $\Gamma \ll 2$ is defined as the mixing region.

\footnotetext{
${ }^{2}$ The source current now has no place to go when the switch is open. However, in the practical circuit implementations the number of polyphase paths is such, that there is always a switch closed at any given time and the current is always routed to a capacitor.
} 


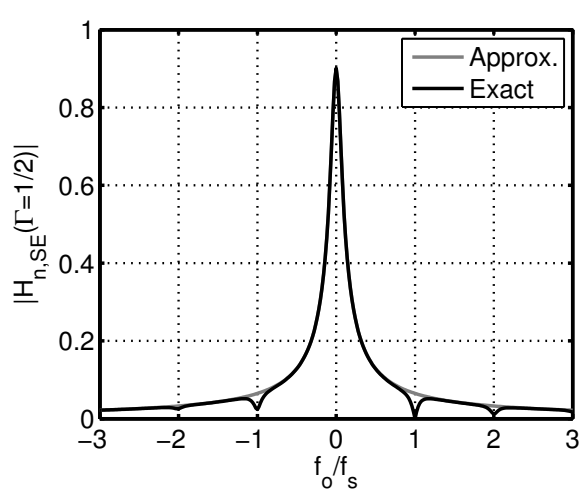

(a)

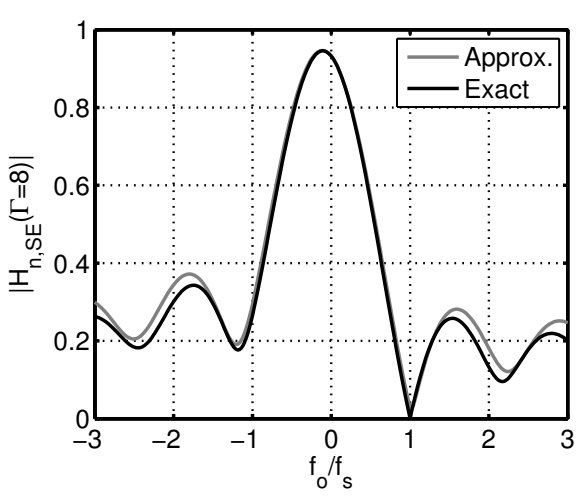

(b)

Figure 3.10: $\operatorname{SE~kernel~} \operatorname{HTF}(n=-1)$ with $25 \%$ duty cycle for the (a) mixer region $(\Gamma=1 / 2)$ and (b) sampling region $(\Gamma=8)$.

Appendix A.3 also derives the following HTF for the mixing region $(\Gamma \ll 2)$ :

$$
H_{n, S E}\left(f_{o}\right) \approx \frac{\operatorname{sinc}(D n)}{1+j \frac{f_{o}}{D f_{r c}}} e^{-j \pi D n}
$$

This equation includes the familiar mixing term from equation (3.4) and a lowpass output filter with bandwidth $D \cdot f_{r c}$.

The accuracy of these approximation is illustrated in Fig. 3.10, which plots exact and approximated HTFs of the mixing and sampling region. Note that the naming of the regions does not reflect the application but merely the similarity in operation, i.e. a sampling region SE kernel can also be used as a downconverter.

For the DI kernel, the same calculation method can be used to get the HTFs. Appendix A.2 derives:

$$
H_{n, D I}\left(f_{o}\right)=P_{D I}\left(f_{o}\right)\left[\frac{1-e^{-j 2 \pi D n}}{j 2 \pi n}+\frac{e^{j 2 \pi\left(\frac{1}{2}-D\right) f_{o} / f_{s}}-1}{j 2 \pi f_{o} / f_{s}} G_{D I}\left(f_{o}-n f_{s}\right)\right]
$$

where:

$$
\begin{gathered}
P_{D I}\left(f_{o}\right)=\frac{\left(1-e^{-j 2 \pi \frac{1}{2} n}\right)}{2} \frac{1}{1+j \frac{f_{o}}{f_{r c}}} \\
G_{D I}\left(f_{i}\right)=-\frac{e^{j 2 \pi D f_{i} / f_{s}}-e^{-2 \pi D f_{r c} / f_{s}}}{e^{j 2 \pi \frac{1}{2} f_{i} / f_{s}}+e^{-2 \pi D f_{r c} / f_{s}}} \frac{1}{1+j \frac{f_{i}}{f_{r c}}} .
\end{gathered}
$$

Compared to $P_{S E}$, the output filter $P_{D I}$ now includes an extra $\left(1-e^{-j 2 \pi \frac{1}{2} n}\right) / 2$, which evaluates to zero for all even $n$ and to unity for all odd $n$. This means that the DI 


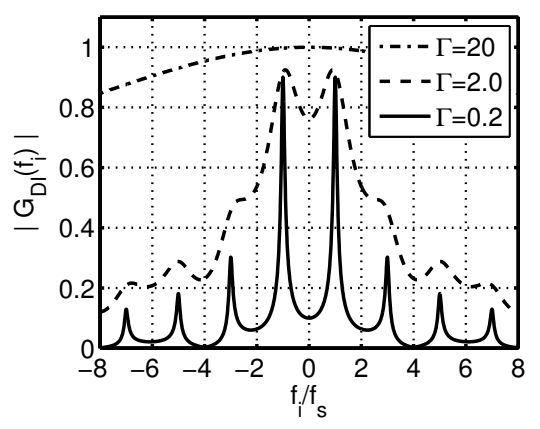

Figure 3.11: Filter $G_{D I}$ plotted for several $\Gamma$ and duty cycle $D=0.25$.

kernel already cancels all even-order harmonics. As is plotted in Fig. 3.11, the input filter $G_{D I}$ now has no spikes at even multiples of the clock frequency, and each spike has double the width.

When the DI kernel mixing region approximation is calculated $(\Gamma \ll 2)$ :

$$
H_{n, D I}\left(f_{o}\right) \approx \begin{cases}\frac{\operatorname{sinc}(D n)}{1+j \frac{f_{o}}{2 D f_{r c}}} e^{-j \pi D n} . & \text {, odd } n \\ 0 & , \text { even } n\end{cases}
$$

it is seen that the bandwidth has doubled with respect to the SE kernel. This is due to the fact that the capacitor is connected to the input twice each clock cycle, thereby halving the effective resistance. The sampling region approximation is given by $(\Gamma \gg 2)$ :

$$
\begin{aligned}
& H_{n, D I}\left(f_{o}\right) \approx
\end{aligned}
$$

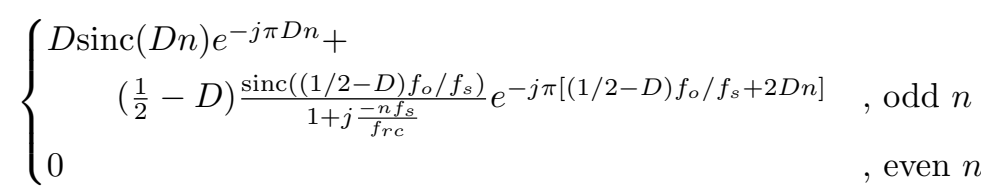

and shows a smaller duty cycle of $\left(\frac{1}{2}-D\right)$ for the holding interval.

Due to the similarities between the SE and DI kernel, the rest of the analysis will concentrate on the SE kernel. All of the derived properties also apply to the DI kernel, when it is taken into account that it has double the bandwidth in the mixing region.

\subsubsection{Polyphase Multi-path Analysis}

Polyphase multi-path systems allow the combination of multiple kernels into a larger system. It will be shown how such a configuration can cancel unwanted harmonics in $H_{n}$, while passing the wanted harmonics. 


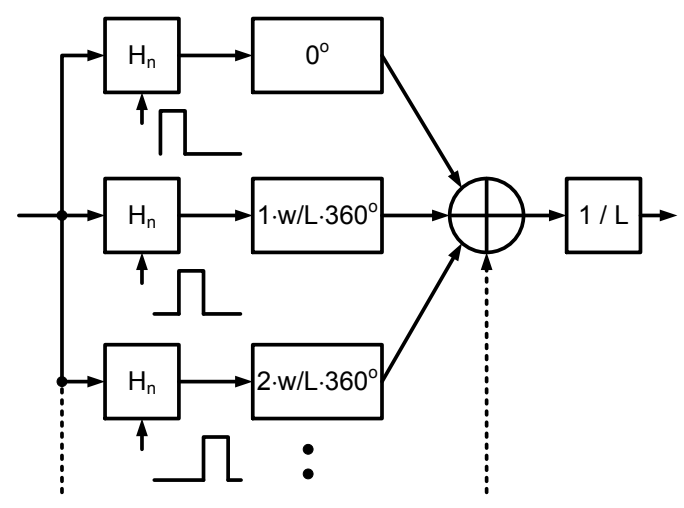

Figure 3.12: Polyphase multi-path system model with $L$ paths.

The generic polyphase multi-path model is shown in Fig. 3.12. A number of $L$ parallel branches, each consisting of an LPTV system with shifted clocking and a fixed LTI phase shift, are summed into a common output node. The basic idea here is that the time shift in the clocking of the LPTV blocks corresponds to different phase shifts for each of the harmonics in the HTF. By then applying a second phase shift on all of the harmonics with an LTI circuit, the combined phase shifted signals at the output node sum up to either zero or $H_{n}\left(f_{o}\right)$.

First, the phase shift of the LPTV blocks is considered. From Fourier analysis, it is known that shifting a signal in time causes a linear phase shift in the frequency domain. Mathematically, if the time shift is $\sigma_{d}$, the Fourier pair of signal $v$ equals:

$$
v\left(t-\sigma_{d}\right) \stackrel{\mathcal{F}}{\leftrightarrow} V(f) e^{-j 2 \pi f \sigma_{d}} .
$$

For an LPTV system with HTFs $H_{n}$, shifting the clocking (and thus the switching moments) by $\sigma_{d}$ has the same effect as shifting both the input and output by $-\sigma_{d}$ :

$$
\begin{aligned}
v_{i}\left(t+\sigma_{d}\right) & \stackrel{\mathcal{F}}{\leftrightarrow} V_{i}\left(f_{i}\right) e^{j 2 \pi f_{i} \sigma_{d}} \\
v_{o}\left(t+\sigma_{d}\right) & \stackrel{\mathcal{F}}{\leftrightarrow} V_{o}\left(f_{o}\right) e^{j 2 \pi f_{o} \sigma_{d}}
\end{aligned}
$$

Such that the frequency response of an LPTV system shifted in time can be expressed as:

$$
\begin{aligned}
V_{o}\left(f_{o}\right) e^{j 2 \pi f_{o} \sigma_{d}} & =\sum_{n=-\infty}^{\infty} H_{n}\left(f_{o}\right) V_{i}\left(f_{o}-n f_{s}\right) e^{j 2 \pi\left(f_{o}-n f_{s}\right) \sigma_{d}} \\
V_{o}\left(f_{o}\right) & =\sum_{n=-\infty}^{\infty} H_{n}\left(f_{o}\right) e^{-j 2 \pi n f_{s} \sigma_{d}} V_{i}\left(f_{o}-n f_{s}\right) .
\end{aligned}
$$

and it is seen that the HTFs get a phase term equal to $e^{-j 2 \pi n f_{s} \sigma_{d}}$ when the switching moments are shifted forward by $\sigma_{d}$ in time. 
For the polyphase multi-path system, it was defined that the parallel branches are switched by a polyphase clock, with clock phases that are distributed uniformly across the clock period. In other words: the $l$-th path has a clock shifted by $\sigma_{d}=l / L \cdot T_{s}$, resulting in a phase shift of $-n \cdot l \cdot 360^{\circ} / L$.

Next, the LTI phase shift is applied, adding a phase shift of $l \cdot w \cdot 360^{\circ} / L$ for the $l$-th path. The $w$ parameter indicates the harmonic index of the first uncanceled harmonic (provided that $w<L$ ), as will be shown shortly. Each path now has the total phase term:

$$
e^{-j 2 \pi n l / L} e^{j 2 \pi w l / L}=e^{-j 2 \pi(n-w) l / L} .
$$

Summing the signals over all paths gives the HTFs of the total system:

$$
\begin{aligned}
H_{n, \text { polyphase }}\left(f_{o}\right) & =\frac{1}{L} \cdot \sum_{l=0}^{L-1} H_{n}\left(f_{o}\right) e^{-j 2 \pi(n-w) l / L} \\
& = \begin{cases}0 & , \frac{n-w}{L} \neq \text { integer } \\
H_{n}\left(f_{o}\right) & , \frac{n-w}{L}=\text { integer }\end{cases}
\end{aligned}
$$

In other words, a polyphase multi-path system passes all frequency shifts with a harmonic index that satisfies:

$$
n=i \cdot L+w \text { where } i=\ldots,-2,-1,0,1,2, \ldots
$$

while the transfer functions for all other frequency shifts are zero. Indeed, the first uncanceled harmonic is the one with index $w$. For example, with $L=2$ and $w=-1$, this results in cancellation for all $n=\ldots,-2,0,2, \ldots$. It can also be seen that for more than two paths $(L>2$ ), either $n$ or $-n$ (or both) are canceled, automatically resulting in image rejection.

Two familiar examples of a polyphase system are the differential mixer in Fig. 3.13a and the Hartley image rejection mixer in Fig. 3.13b ${ }^{3}$. The scaling factor of $1 / L$ in Fig. 3.12 is a normalization factor, and might not always be present, but its absence will not modify the fundamental harmonic canceling properties of the multi-path polyphase system.

\subsubsection{Noise}

Up to this point, the analysis has been concerned with the transfer function of deterministic signals. Of additional interest is the behavior of these LPTV systems

\footnotetext{
${ }^{3}$ In an image-reject receiver the I and Q outputs are usually digitized separately and summed in the digital domain. Compared to analog summing, the resulting fundamental system image rejection is equivalent.
} 


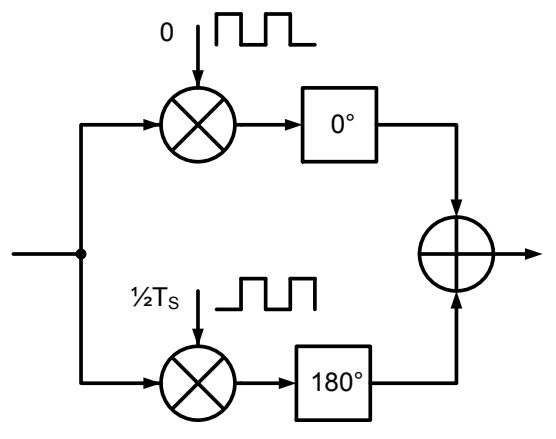

(a)

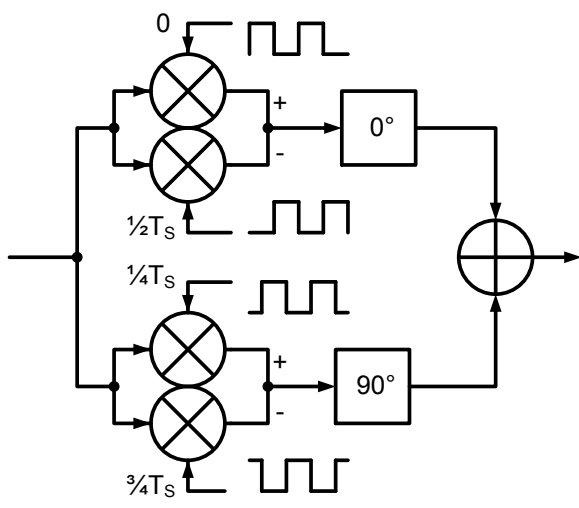

(b)

Figure 3.13: Polyphase multi-path example with (a) $L=2, D=50 \%$ and (b) $L=4$, $D=50 \%$.

with random, noisy inputs. For random signals, the relation between the input power spectral density (PSD), $N_{i}$, and output PSD, $N_{o}$, in LPTV systems is [52]:

$$
N_{o}\left(f_{o}\right)=\sum_{n=-\infty}^{\infty}\left|H_{n}\left(f_{o}\right)\right|^{2} N_{i}\left(f_{o}-n f_{s}\right) .
$$

Compared to the HTF for deterministic signals in (3.2), random signals are also shifted in frequency and summed together, but now according to the absolute HTF squared, as the equation is expressed in terms of power.

It will now be derived how the differences in filtering between the mixer and sampling regions expresses itself in the intrinsic noise PSD of the kernel. Returning to the Thevenin model of the SE kernel in Fig. 3.9a, it can be seen that the intrinsic input noise is solely due to the resistor $R$. Its equivalent noise voltage source can directly be considered to form the input voltage for the kernel, so that the doublesided PSD at the input is expressed as:

$$
N_{i}=2 k T R
$$

Applying equation (3.25) gives the double-sided PSD as:

$$
N_{o}\left(f_{o}\right)=2 k T R \sum_{n=-\infty}^{\infty}\left|H_{n}\left(f_{o}\right)\right|^{2} .
$$

The evaluation of this expression requires us to sum the absolute HTF squared over all harmonics, which proves to gives mathematical difficulties for the exact HTF of the kernels. 


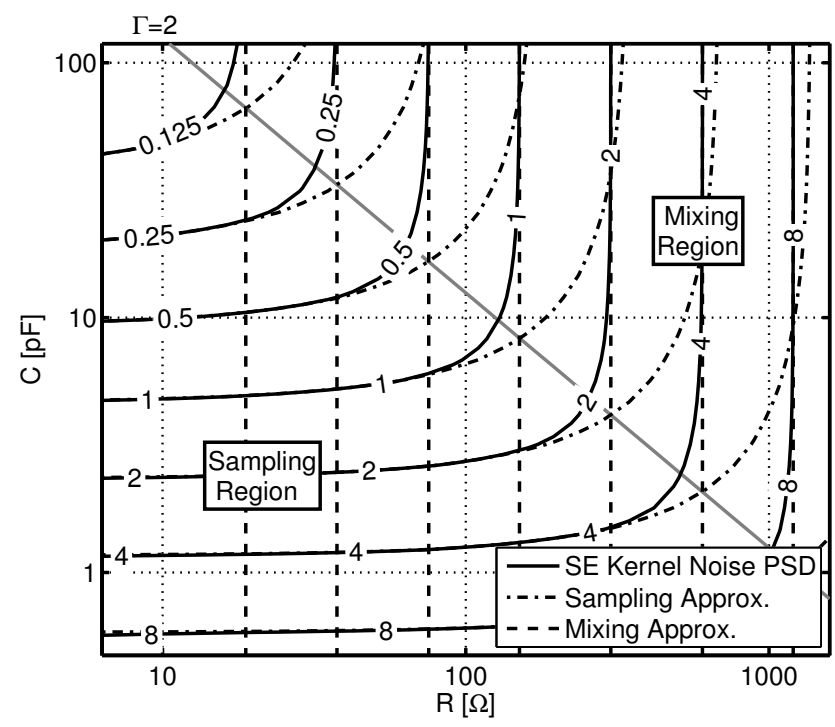

Figure 3.14: Contour plot of the SE kernel noise one-sided PSD ( $f_{o}=0 \mathrm{~Hz}, f_{s}=100$ $\mathrm{MHz}, D=25 \%$ ) $\left[10^{-17} \mathrm{~V}^{2} / \mathrm{Hz}\right]$ calculated by substituting HTFs (3.8) into (3.25), and the sampling (3.29) and mixing (3.28) region approximation.

Instead, the evaluation for the mixing and sampling region is performed. The precise derivation is given in appendix A.4 and involves some integrals and sums. Evaluation for DC output frequency $\left(f_{o}=0\right)$ gives the following expression of the output noise PSD for the mixing region:

$$
N_{o}(0)=2 k T R \frac{1}{D} \quad, \Gamma \ll 2 .
$$

Which is equal to the PSD of a resistor $R$, but increased by the inverse of the duty cycle. This can be attributed to the folding of white resistor noise by the mixing harmonics. Moreover, the PSD for the sampling region gives:

$$
N_{o}(0)=2 k T\left[(2-D) R+\frac{(1-D)^{2}}{2 f_{s} C}\right] \quad, \Gamma \gg 2 .
$$

This equation is very similar to the one derived in a time-domain analysis of sampleand-hold circuits [45], with an identical second term and slightly modified first term. The first term is associated with the switch noise directly showing at the output during the track interval and is more accurate in this case. The second term is dominant and represents the sampled noise at the switching moment which is held on the capacitor during the hold interval and is identical to [45].

To show the accuracy of these calculations, Fig. 3.14 plots a contour of the approximate mixing region PSD from (3.28) and sampling region PSD from (3.29) in 
dashed lines, and the 10000 term evaluation of the exact PSD using (3.25) and (3.8) in solid lines. In the limit, the approximations converge to the exact solution, and simulations in Spectre RF confirm the results ${ }^{4}$.

The figure also shows that (3.28) and (3.29) are equal for $\Gamma=2$, i.e. the dashed lines intersect on the grey $\Gamma=2$ line. This motivates the choice for this particular border between the two regions.

An additional property that can be extracted from the figure is that for the mixing region, the noise PSD is determined mostly by the resistance $R$, while for the sampling region the noise PSD is determined mostly by the capacitance $C$. When dimensioning a circuit for noise, this difference between the two regions is crucial.

As a final step, appendix A.4 evaluates (3.28) and (3.29) for non-zero $f_{o}$, so that the output noise PSD can be integrated to give the total noise power:

$$
P_{o}=\int_{-\infty}^{\infty}\left|N_{o}\left(f_{o}\right)\right|^{2} d f_{o}
$$

We find that for both the regions, the total integrated noise evaluates to:

$$
P_{o}=\frac{k T}{C}
$$

which is well known for sampled-data systems and is shown here to also hold for the mixing regime.

For a polyphase multi-path system, the same analysis can be applied, but now with the appropriate polyphase HTFs (3.23). But, there is a shorter path to derive the impact of polyphase combination without re-evaluating the sums and integrals.

The crux of the derivation consists of the realization that the source resistance $R_{s}$ is shared between polyphase paths, but is never connected to multiple paths at the same time. Assuming white input noise, the noise voltage at one time is uncorrelated to the noise voltage at a different time. Therefore, the noise powers of the polyphase paths are completely uncorrelated.

As a consequence, the effect of the summing node and scaling factor $1 / L$ in Fig. 3.12 is the averaging of the output noise:

$$
N_{o, \text { polyphase }}=N_{o, \text { kernel }} / L
$$

where $N_{o, \text { kernel }}$ is the output PSD of a single kernel.

On the other hand, a deterministic input signal results into completely correlated output signals on each of the polyphase branches. The averaging effect of the summing node and scaling factor now results in the same output signals compared to a single kernel.

\footnotetext{
${ }^{4}$ Simulation in Spectre RF of especially the sampling region noise PSD is very involving, due to the large number of harmonics that have to be evaluated by the simulator. For an excellent treatment on this subject, consult [53].
} 


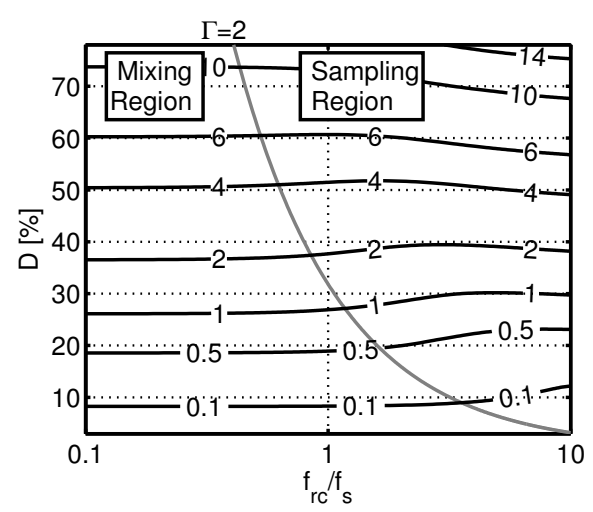

(a)

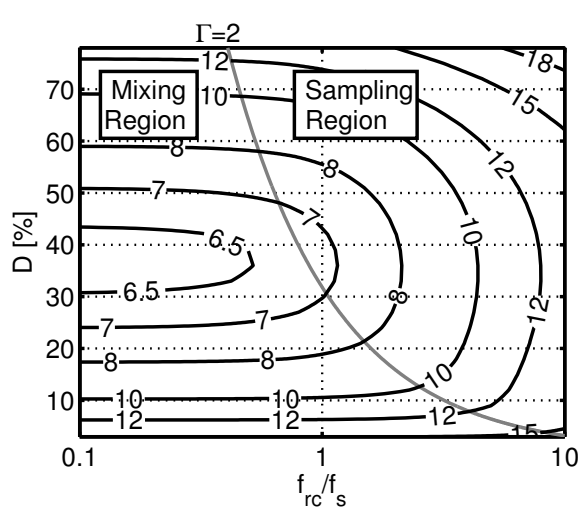

(b)

Figure 3.15: SE kernel (a) conversion loss in $\mathrm{dB}$ and (b) NF in dB for downconversion $(w=-1)$.

Thus, the net effect of polyphase multiphase combining on the circuit noise is an increase in SNR equal to the number of paths $L$. It can be understood that the cancellation of harmonics reduces the amount of noise folding.

\subsubsection{Application to Low-Noise Passive Mixers}

This section started with the desire to build low-loss, low-noise passive mixers, for use in the mixer-first architecture. The first parameter to consider is the conversion gain, $C G_{w}\left(f_{o}\right)$, defined as the HTF with harmonic index $w$ :

$$
C G_{w}\left(f_{o}\right) \equiv H_{n=w}\left(f_{o}\right)
$$

From a plot of the SE kernel conversion loss as a function of the duty cycle and RC bandwidth (Fig. 3.15a), it is evident that a lower duty cycle results in a lower conversion loss. Note that the RC bandwidth of the kernel has a rather small effect on the conversion loss.

The second parameter to consider is the single side band (SSB) noise figure, defined as the ratio between the input and output SNR:

$$
\begin{aligned}
F_{w}\left(f_{o}\right) & =\frac{\mathrm{SNR}_{\text {in }}}{\mathrm{SNR}_{\text {out }}}=\frac{N_{o}\left(f_{o}\right)}{N_{i}\left(f_{o}-w f_{s}\right)} \cdot \frac{1}{\left|C G_{w}\left(f_{o}\right)\right|^{2}} \\
N F_{w}\left(f_{o}\right) & =10 \log \left(F_{w}\left(f_{o}\right)\right),
\end{aligned}
$$

The input noise PSD $N_{i}$ and output noise PSD $N_{o}$ are given by equation (3.26) and (3.27) respectively. Together with the conversion gain, a plot of the kernel NF (Fig. $3.15 \mathrm{~b}$ ) can be made, and it is seen that the mixing region has an advantage in noise 


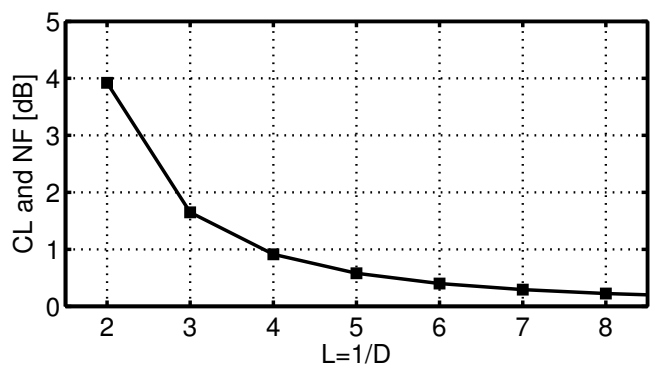

Figure 3.16: Mixing region conversion loss and minimum noise figure due to folding.

figure over the sampling region for equal duty cycle. Additionally, there is an optimum in noise figure a duty cycle of around $37 \%$.

One additional thing must be considered though. The loop resistance $R$ is in fact the sum of source resistance $R_{s}$ and switch-on resistance $R_{o n}$. Only the first represents the actual input noise, while the second is noise added by the circuit itself. In the total noise figure, the input noise is therefore a factor of $R_{s} / R_{s}+R_{o n}$ smaller.

Applying this to equation (3.34) gives the same expression for the kernel noise figure as before, but now with an extra factor:

$$
F_{w}\left(f_{o}\right)=\underbrace{\left(1+\frac{R_{o n}}{R_{s}}\right)}_{\mathrm{A}} \cdot \underbrace{\frac{\sum_{n=-\infty}^{\infty}\left|H_{n}\left(f_{o}\right)\right|^{2}}{\left|H_{w}\left(f_{o}\right)\right|^{2}}}_{\mathrm{B}} .
$$

The A factor is added and corresponds to the Linear Time Invariant (LTI) noise figure for the SE kernel with closed switch and no periodic switching at all. The B term represents the noise folding due to the circuit switching, plotted in Fig. 3.15b. Therefore, for calculating the NF, the LPTV kernel analysis can be done with the total resistance $R$ and the effect of $R_{o n}$ can be factored in afterwards.

Continuing by considering the results from the polyphase noise analysis, it is found that the noise figure decreases when the number of polyphase paths is increased. The limit on the number of paths is the requirement that the clock is non-overlapping, so for optimum noise figure we have to "fill the phases", and set the number of paths $L$ to:

$$
L=\frac{1}{D}
$$

If we furthermore use the approximate transfer function for the mixing region, the noise factor can be expressed as:

$$
F=\left(1+\frac{R_{\text {on }}}{R_{s}}\right) \cdot \frac{1}{|\operatorname{sinc}(D)|^{2}} .
$$


The noise contribution due to the switches can be minimized by lowering the switchon resistance ${ }^{5}$ and the contribution due to the noise folding can be minimized by decreasing the duty cycle (which increases the number of polyphase paths). As the conversion los of a single kernel is equal to $\operatorname{sinc}(D)$, we find that for zero switch-on resistance the fundamental minimum conversion gain and minimum noise figure of a polyphase passive mixer are equal to:

$$
C G_{\text {kernel }}[d B]=-N F_{\min }[d B]=-20 \cdot \log (\operatorname{sinc}(D))
$$

So although a single kernel has on optimum in noise for $37 \%$ duty cycle, a completely filled polyphase system of kernels has lower noise for smaller duty cycles.

Plotting the conversion gain and noise figure for the completely filled polyphase system in Fig. 3.16, it can be observed that the step from 2 paths and $50 \%$ duty cycle, to 4 paths and $25 \%$ duty cycle delivers a big improvement. Conveniently this requires four polyphase paths, which can be arranged as a differential In-phase and Quadrature output for image rejection and vector modulation. The improvements with a further increase of the number of paths is less pronounced.

To demonstrate the high SFDR of such a mixer-first receiver with a DI kernel, $25 \%$ duty cycle switched-RC mixer, the next section presents a chip design in advanced CMOS.

\subsection{Implementation in 65-nm CMOS}

Figure 12.4.2 shows the proposed mixer-first direct conversion architecture with a 4-phase $25 \%$ duty cycle differential mixer and feedback amplifiers at IF. A completely differential design cancels out second order distortions, ensuring that third order intermodulation harmonics dominate the linearity performance.

\subsubsection{Mixer and Feedback Amplifier}

The bandwidth $B W$ is set by the DI kernel capacitance:

$$
C=\frac{D}{\pi R \cdot B W}
$$

where the $B W$ must be smaller than $\pi D / f_{s}$ to ensure that the kernel operates in the mixing region. The resistance for the DI mixer kernel consists of the $5 \Omega$ switch on-resistance added to the $50 \Omega$ source impedance, which together with $65 \mathrm{pF}$ on-chip capacitance results in $22 \mathrm{MHz}$ of mixer bandwidth. The switch resistance increases the mixer NF to $1.9 \mathrm{~dB}$ and limits the IIP3 to $26 \mathrm{dBm}$.

\footnotetext{
${ }^{5}$ The switch-off resistance is rarely a limiting factor in practical MOSFET switches.
} 


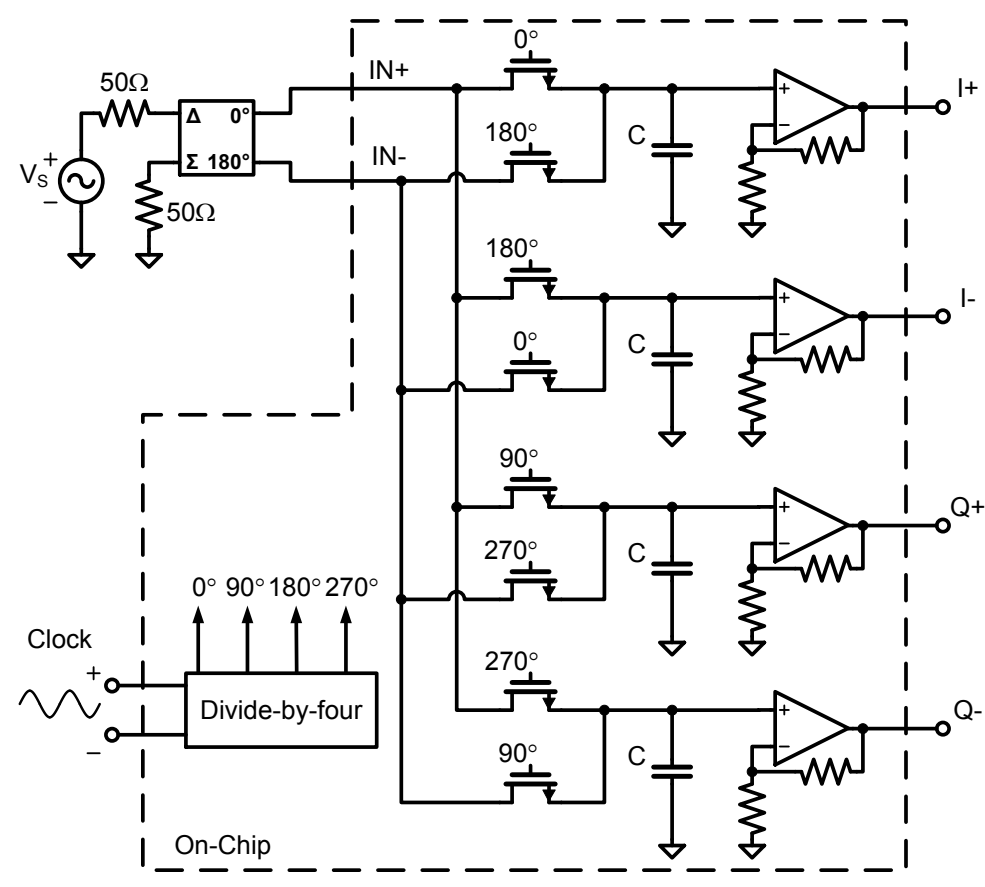

Figure 3.17: Direct conversion receiver architecture.
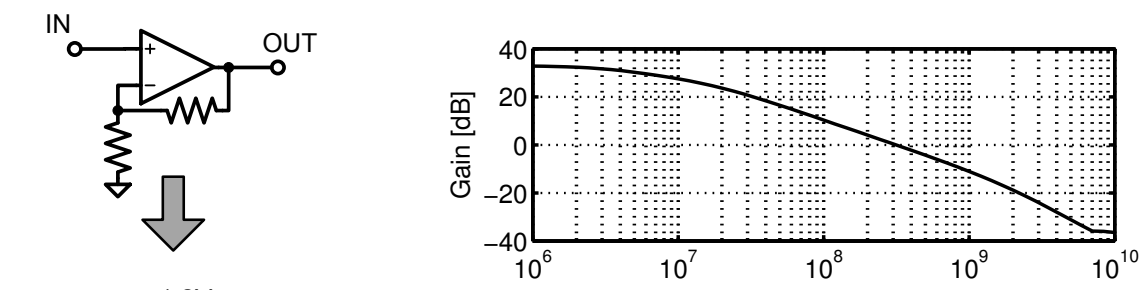

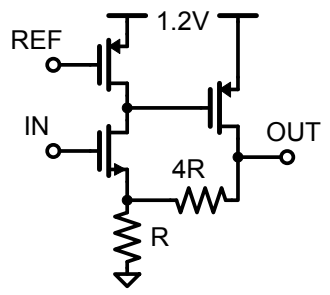

(a)

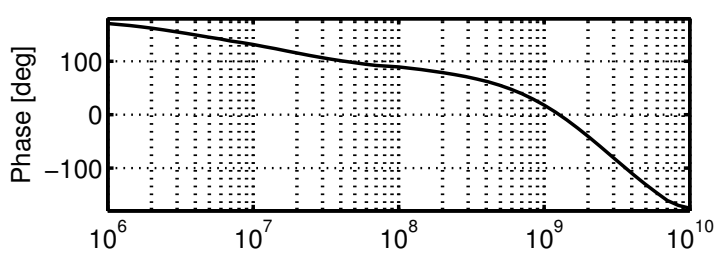

(b)

Figure 3.18: Negative feedback amplifier (a) schematic with (b) simulated loop gain and phase. 


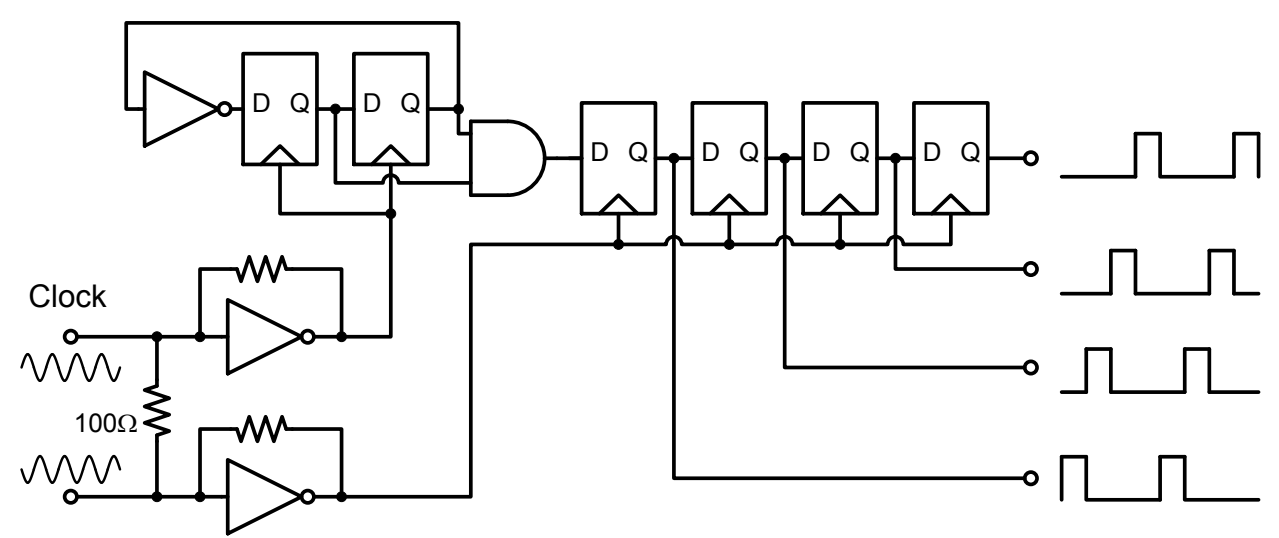

Figure 3.19: Clock divider.

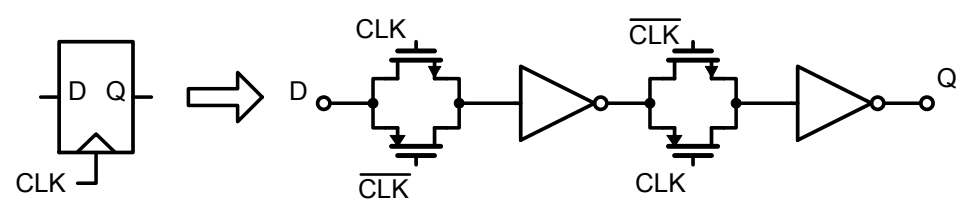

Figure 3.20: Flipflop.

Fig. 3.18a shows the feedback amplifier. The NMOS with current source load provides most of the loop gain. It is followed by a PMOS common-source stage driving the resistive feedback network with feedback to the source of the NMOS. The 4 to 1 ratio in the feedback network results in a $13.5 \mathrm{~dB}$ voltage gain and transistor sizes result in a $3 \mathrm{~dB}$ amplifier NF. The simulated loop gain shown at the right side of Fig. 3.18b is about $32 \mathrm{~dB}$ and rolls of to $29 \mathrm{~dB}$ at $9 \mathrm{MHz}$.

The decreasing loop gain diminishes the linearizing effect of the feedback at higher IF frequencies, resulting in a lower IIP3 for out-of-band interferers. This effect is compensated by the increasing conversion loss of the mixer for higher IF frequencies, resulting in a minimum system IIP3 of $12.5 \mathrm{dBm}$.

In this proof-of-concept design the amplifier is kept simple and its IIP3 is still dominant for the system linearity.

\subsubsection{Clock Generation}

For correct operation of the mixer, a $25 \%$ duty cycle 4-phase rectangular clock is required. As a rectangular clock has a much higher harmonic content than a sinusoidal one, it is unpractical to transport such a clock on a PCB. Moreover, RF signal generators only output a single-phase sinusoidal signal and microwave passive components 


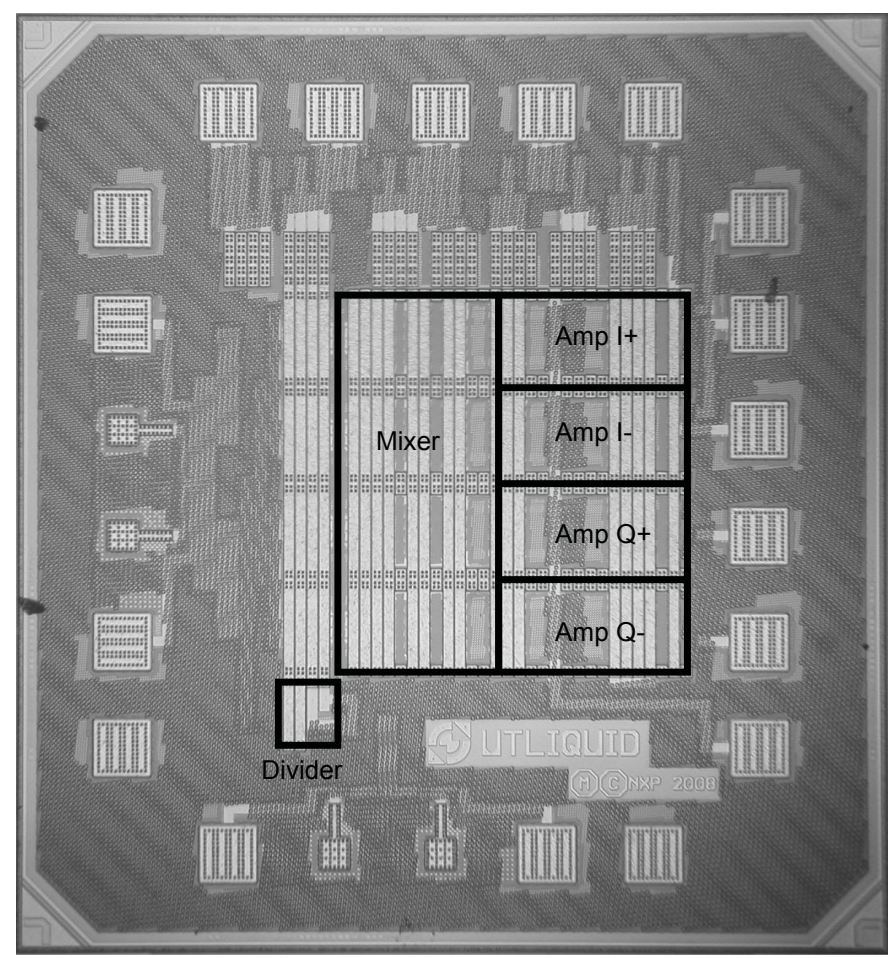

Figure 3.21: Photograph of the $0.95 \mathrm{~mm}$ by $1.1 \mathrm{~mm}$ die.

are not able to generate four accurate phases from this signal. The solution is to apply an external sinusoidal clock at four times the LO frequency and divide-by-four on-chip to generate a 4 -phase $25 \%$ duty cycle clock.

The clock divider is plotted schematically in Fig. 3.19. The external differential sinusoidal clock at 4 times the LO frequency is first clipped by two self-biased inverters to serve as a master clock for the rest of the circuit. A loop of two flipflops and an inverter serves as a divide-by-four. The AND gate combines two $50 \%$ duty cyle pulses from this loop and combines them to produce a $25 \%$ duty cycle clock. The required four phases are then created by means of a four-flipflop shift register.

Each flipflop is implemented by dynamic transmission gate logic, Fig. 3.20. During the first clock phase, the first transmission gate turns on and the input is put on the gates of the first inverter, which acts as a memory device. During the second clock phase, the second transmission gate turns on and the voltage on the internal node is passed on towards the output inverter. This logic omits the feedback inverters and relies on the fact that the data is updated regularly, before charge on the internal nodes leaks away.

As the flipflops are completely dynamic, the dissipated power from the supply will 


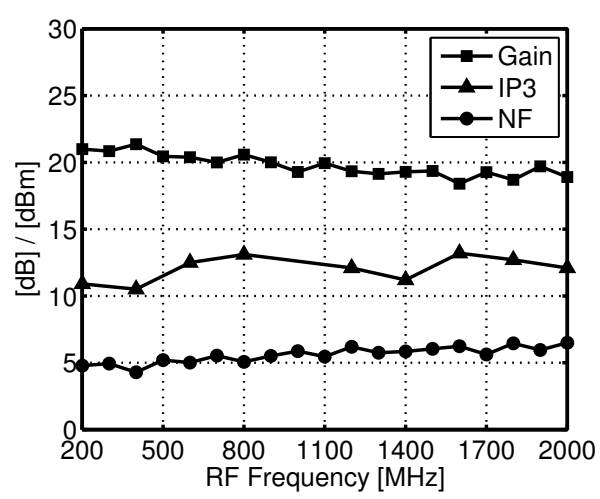

(a)

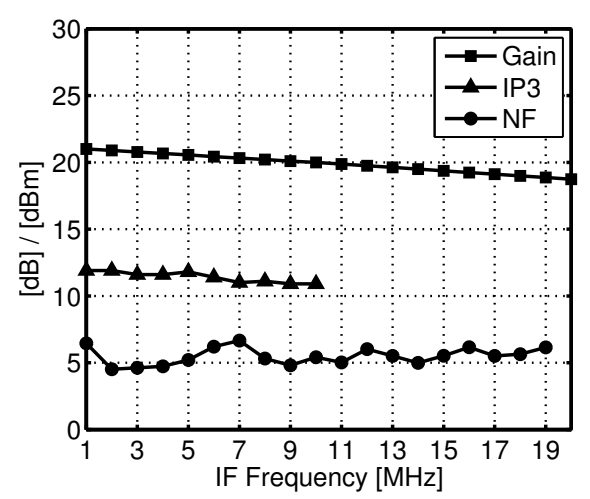

(b)

Figure 3.22: Measured Gain, NF and in-band IIP3 versus (a) RF and (b) IF frequency.

depend linearly on the switching frequency, resulting in a low power for low speeds and a high power for high speeds.

\subsubsection{Measurements}

The circuit is fabricated in a standard 65-nm LP CMOS technology. Fig. 3.21 shows a die micrograph, where the die size is $0.95 \mathrm{~mm}$ by $1.1 \mathrm{~mm}$.

At the RF input of the circuit, a microwave hybrid is used to perform the sideband single-ended to differential conversion. As will be explored further in section 5.1.2, the input of the mixer is fundamentally unmatched to the source impedance, presenting a high input impedance in the pass band. This results in a passive $6 \mathrm{~dB}$ of voltage gain at the input [54], but leaves a difficult design for a possible RF band filter, while presenting reflections when further impedance mismatches are present in the measurement setup.

Fig. 3.22b shows the measured performance figures at different IF frequencies and an RF frequency of $500 \mathrm{MHz}$ as markers, while the simulated figures are shown as lines. The measured voltage conversion gain rolls off from $22 \mathrm{~dB}$ to $20.5 \mathrm{~dB}$ due to the mixer IF pole. The NF is lower than $6.5 \mathrm{~dB}$ and increases at lower IF frequencies due to flicker noise of the amplifiers and at higher IF frequencies due to a decrease in gain. The measured minimum IIP3 is $+11 \mathrm{dBm}$.

Fig. 3.22a shows the measured performance figures at different RF frequencies and an IF frequency of $5 \mathrm{MHz}$ as markers, while the simulated figures are shown as lines. The clock divider limits the RF frequency to $2 \mathrm{GHz}$. The phase mismatch between outputs was measured to be below 2 degrees. LO Radiation occurs at the input at multiples of the clock frequency with a maximum level of $-65 \mathrm{dBm}$. The 
Table 3.1: Performance Summary

\begin{tabular}{|c|c|}
\hline Technology & $65-\mathrm{nm}$ CMOS \\
\hline Active Area & $0.13 \mathrm{~mm}^{2}$ \\
\hline RF frequency band & $0.2-2.0 \mathrm{GHz}$ \\
\hline Power consumption & $64-100 \mathrm{~mW}{ }^{a}$ \\
\hline IF -3 dB bandwidth & $25 \mathrm{MHz}$ \\
\hline Gain & $19 \mathrm{~dB}$ \\
\hline NF & $6.5 \mathrm{~dB} \mathrm{DSB}$ \\
\hline In-band IIP3 & $11 \mathrm{dBm}$ \\
\hline In-band IIP2 & $65 \mathrm{dBm}$ \\
\hline SFDR in 1 MHz BW & $79 \mathrm{~dB}$ \\
\hline
\end{tabular}

${ }^{\mathrm{a}}$ of which $60 \mathrm{~mW}$ is static power.

clock divider consumes $7 \mathrm{~mW}$ and the mixer plus amplifiers consume $60 \mathrm{~mW}$ from a $1.2 \mathrm{~V}$ supply.

Table 3.1 summarizes the achieved performance. Applying the measured NF and IIP3 to equation (3.1) results in a SFDR of $79 \mathrm{~dB}$ in $1 \mathrm{MHz}$ bandwidth. In comparison, the commercially available Tektronix RSA2200A spectrum analyzer provides $80 \mathrm{~dB}$ of SFDR in the same bandwidth.

\subsection{Conclusions}

This chapter has analyzed the use of switched-RC donwconverters in a mixer-first receiver architecture. It was shown that a broad range of switched-RC samplers and mixers can be understood to be an assembly of simple kernels with polyphase clock timing. A unified frequency domain description was derived, highlighting the similarity in operation of these circuits. Moreover, it was shown that there are two distinct operating regions of the kernels, depending on the loop bandwidth with respect to the clock frequency and duty cycle.

Analysis shows that using a $25 \%$ duty cycle switched-RC kernel with low loop bandwidth and a 4-phase clock provides a low fundamental conversion loss and noise figure, while being compatible with a I/Q image-reject architecture. This configuration, together with baseband feedback amplifiers, is implemented in a $65 \mathrm{~nm}$ CMOS implementation and achieves $79 \mathrm{~dB}$ of SFDR in $1 \mathrm{MHz}$ bandwidth, which is in the range of commercial spectrum analyzers. 


\section{Chapter 4}

\section{Approximate-Sine Vector Modulator Beamforming}

In the previous chapter, the highly linear mixer-first architecture with passive mixing has been introduced to serve as a downconverting front-end. In phased arrays, the key additional component to be considered is the phase shifter, which function is to align the incoming antenna signals in phase. For this circuit, additional design trade-offs besides spurious free dynamic range need to be made, such as phase shift resolution, amplitude error and frequency range. A straight-forward design approach that uses classical filter design to build the correct phase response can be unpractical for CMOS integration, due to the poor integration of coils on-chip and limited operating frequency of active filters.

Alternatively, different ways of producing a phase shift have been introduced, such as injection locking [55], LO phase selection [56] and vector modulation [57][25][19]. Each approach has its own strengths and weaknesses, but they all share the property that signals are processed in the continuous-time domain. With CMOS technology being optimized for digital processing, which takes place in the discrete-time domain, there is potentially a lot to be gained in implementing discrete-time phase shifters. To this end, this chapter presents a phased-array architecture based on a switchedcapacitor vector modulator that operates in the discrete-time domain.

\subsection{Switched-Capacitor Phase Shifter}

In this section we will introduce the vector modulator for performing the phase shift and amplitude control in a phased array, and show how it can be implemented with switched-capacitor circuits. 


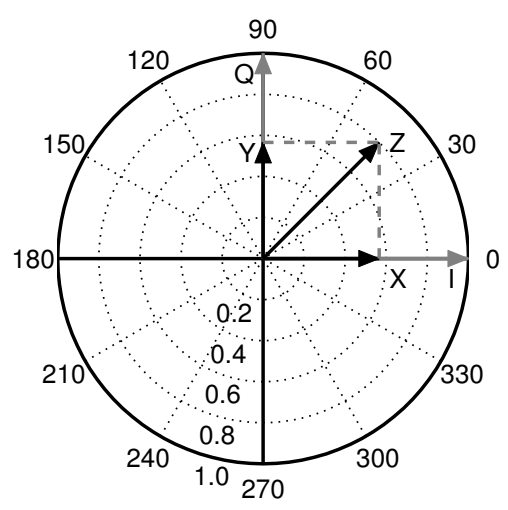

(a)

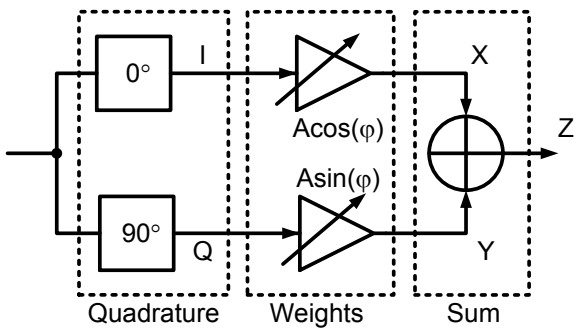

(b)

Figure 4.1: (a) Phasor diagram illustrating the principle of a vector modulator and (b) vector modulator block diagram.

\subsubsection{Vector Modulator Principle}

The vector modulator type of phase shifter is based on the trigonometric relation:

$$
\sin (\omega t+\varphi)=\sin (\varphi) \cdot \cos (\omega t)+\cos (\varphi) \cdot \sin (\omega t)
$$

This equation states that a signal with phase $\varphi$ can be decomposed in a contribution with no phase shift (the $\cos (\omega t)$ term) and a contribution with a fixed $90^{\circ}$ phase shift (the $\sin (\omega t)$ term).

This principle is further outlined in the phasor diagram in Fig. 4.1a, spanned by the real and imaginary axis. The vector to a point $\mathrm{Z}$ in the phasor diagram corresponds to the resultant signal: a phase shifted and amplitude scaled version of the input. $\mathrm{Z}$ can be decomposed into a contribution on the real axis (X vector) and imaginary axis (Y vector) with different lengths according the desired amplitude $A$ and phase $\varphi$ :

$$
\begin{aligned}
& \vec{Z}=\vec{X}+\vec{Y} \\
& \vec{X}=\vec{I} \cdot A \cos (\varphi) \\
& \vec{Y}=\vec{Q} \cdot A \sin (\varphi)
\end{aligned}
$$

Thus, $\mathrm{X}$ is a scaled version of $\mathrm{I}$, the original In-phase input signal and $\mathrm{Y}$ is a scaled version of $\mathrm{Q}$, the Quadrature (90 ${ }^{\circ}$ out-of-phase) version of the input signal. The steps in forming the amplitude-scaled, phase-shifted output $\mathrm{Z}$ from the input are given in the block diagram in Fig. 4.1b. First, In-phase and Quadrature versions of the input are obtained. Then each is weighted to $\mathrm{X}$ and $\mathrm{Y}$ respectively with two variable gain blocks. Finally, the output $\mathrm{Z}$ is formed by summing $\mathrm{X}$ and $\mathrm{Y}$. 


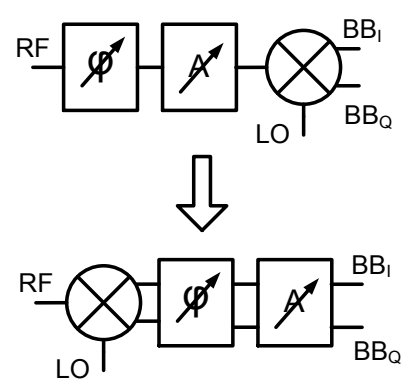

(a)

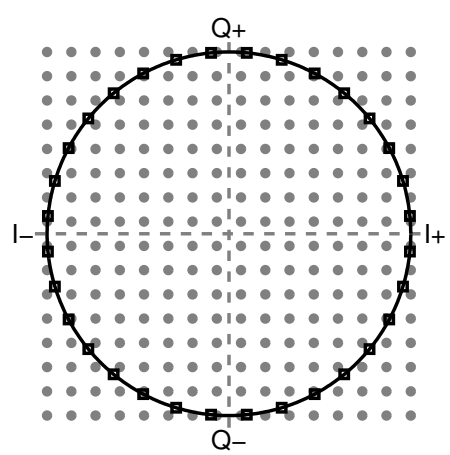

(b)

Figure 4.2: (a) IF phase shifting equivalency to RF phase shifting and (b) mapping onto uniform vector modulator weights.

One way of obtaining the I and Q signal is to include downconversion into the vector modulator. As was derived in section 2.2.2 in transformation A3, the phase shift for beamforming can be moved from the RF port to the IF port of the mixer without affecting the beam pattern. Often, a quadrature mixer with I/Q output is already present in receiver architectures for image rejection purposes, and it can directly be used as input for the vector modulator. Any amplitude scaling at RF can be similarly moved to IF, as is illustrated in Fig. 4.2a.

Furthermore, signals at baseband are often implemented differentially to reject common mode disturbances. From Fig. 4.1a it is apparent that the negative I and Q are also needed to address all four quadrants of the phasor diagram and get a full $0^{\circ}$ to $360^{\circ}$ phase shift range. This can be easily achieved by interchanging the differential signal lines at baseband.

\subsubsection{Sine/Cosine Approximation for Non-Uniform Weighting}

For the beam steering it was concluded that uniform phase steps are preferred (2.14), which require the lengths of the $\mathrm{X}$ and $\mathrm{Y}$ vector to vary according to the sine and cosine of the required phase shift (4.2). Implementing such a variable gain in a robust fashion can be managed in several ways.

With binary weighted parallel amplifier stages, it is straightforward to achieve a uniform quantization of the weights. This is the approach that is predominantly used in vector modulator designs, but it comes at a cost. As Fig. 4.2b illustrates, if the number of quantization steps is insufficient, the desired phase points fall in between implemented weight points. In order to get the required accuracy, a finer quantization 


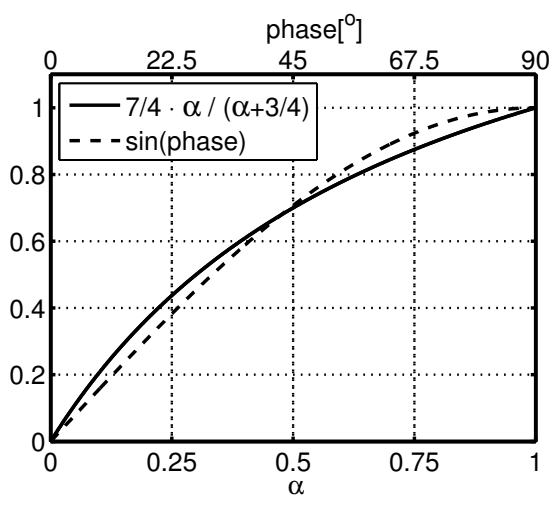

(a)

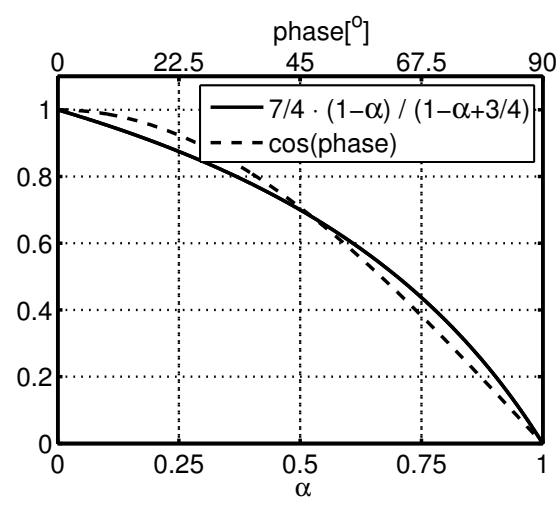

(b)

Figure 4.3: Rational (a) sine and (b) cosine approximation.

has to be applied, leading to many weight points being unused. From an efficiency point of view, this is undesirable and leads to complications in the circuit and layout design.

An approach to reduce the number of quantization steps, is to produce nonuniform quantization which mimics the sine and cosine distribution. To that end, it is proposed to implement an approximation to the sine function with the rational function:

$$
\sin \left(\alpha \cdot \frac{\pi}{2}\right) \approx \frac{7}{4} \frac{\alpha}{\alpha+3 / 4} \quad \alpha=[0,1]
$$

which is plotted in Fig. 4.3a. The $\alpha$ parameter ranges between zero and unity, corresponding to phase shifts from $0^{\circ}$ to $90^{\circ}$ degrees. The factor $3 / 4$ in the denominator is chosen to put the crossover point halfway, in order to minimize the approximation errors. The fraction is scaled to unity gain for $\alpha=1$ by including the factor $7 / 4$. The same function can be used to approximate the cosine, by substituting $\alpha$ with $1-\alpha$ (Fig. 4.3b):

$$
\cos \left(\alpha \cdot \frac{\pi}{2}\right)=\sin \left(\frac{\pi}{2}-\alpha \cdot \frac{\pi}{2}\right) \approx \frac{7}{4} \frac{(1-\alpha)}{(1-\alpha)+3 / 4} .
$$

The elegance of this approximation is that the rational part fits naturally on a voltage divider with variable impedance $\alpha$ and fixed impedance $3 / 4$. The implementation with a 2-phase switched-capacitor circuit is shown in Fig. 4.4a. In the first phase, variable capacitor $\alpha C$ is charged to voltage $V_{I N}$, creating charge $Q_{1}=\alpha C \cdot V_{I N}$. At the same time, a fixed capacitor $3 / 4 C$ is emptied of its charge. In the second phase, the capacitors are connected together and charge redistributes until the capacitor voltages are equal. The charge for the second phase can be expressed as $Q_{2}=(\alpha C+3 / 4 C) \cdot V_{O U T}$. Since charge is conserved, the transfer function evaluates 


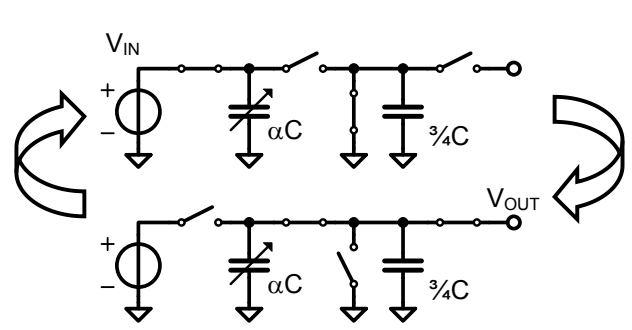

(a)

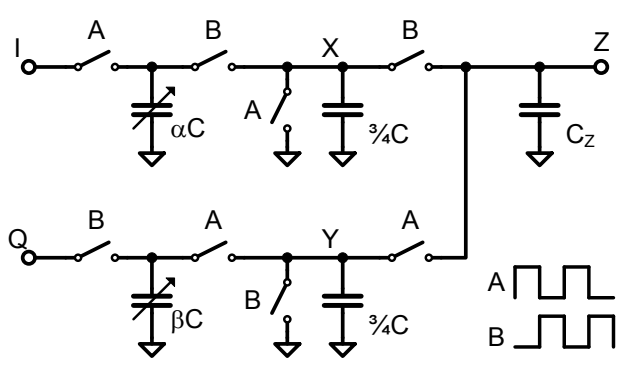

(b)

Figure 4.4: (a) Switching pattern of the charge redistribution principle and (b) the full sine plus cosine approximation circuit.

to the required fraction as a function of $\alpha$ :

$$
\frac{V_{I N}}{V_{\text {OUT }}}=\frac{\alpha}{\alpha+3 / 4}
$$

with a maximum transfer function of $4 / 7$ for $\alpha=1$. Note that as long as the voltages are allowed to settle, the transfer function only depends on capacitor ratios, which are accurately defined. Moreover, with this rational approximation, the required uniform steps in phase correspond to uniform steps in alpha, and thus in uniform steps in capacitance. A simple binary scaled capacitor bank can therefore be used to implement the variable capacitance, with sine and cosine related transfer functions as a result.

As a final step, the $\mathrm{X}$ and $\mathrm{Y}$ vector have to be summed into $\mathrm{Z}$. As the two-phase charge redistribution circuit produces an output for half the time, it is natural to interleave the processing of $\mathrm{X}$ and $\mathrm{Y}$ and de-interleave at $\mathrm{Z}$ with the configuration in Fig. 4.4b. At the output, during clock phase A the $\mathrm{Y}$ signal is present and during clock phase B the X signal is present. After low pass filtering, these voltages will be effectively averaged. The effect of interleaving is essentially a multiplication with a window function in the time domain. Such an operation represents a convolution of the signal with a sinc-scaled comb function in the frequency domain. In other words, the signal is folded around the LO harmonics. The low-pass filtering, which can already be achieved by the channel selection filter, removes these folded contributions and reconstructs the baseband part of the signal.

For uniform phase steps with unity amplitude, $\alpha$ is set to $\varphi \cdot 2 / \pi$ and $\beta$ to $1-\alpha$. To avoid phase points from two quadrants overlapping on the real or imaginary axis, $\alpha$ and $\beta$ are not quantized between 0 and 1 , but between $1 / 16$ and $15 / 16$. The resulting constellation for 32 phase steps is shown in Fig. 4.5, together with the systematic phase and gain error. 

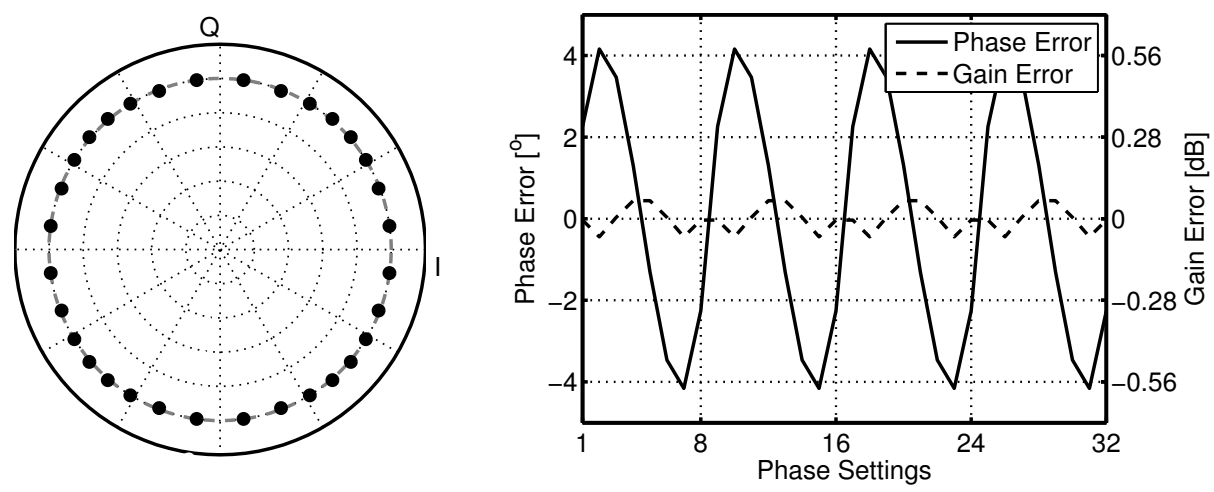

Figure 4.5: Vector modulator phase and gain error for: $C_{Z}=0$.
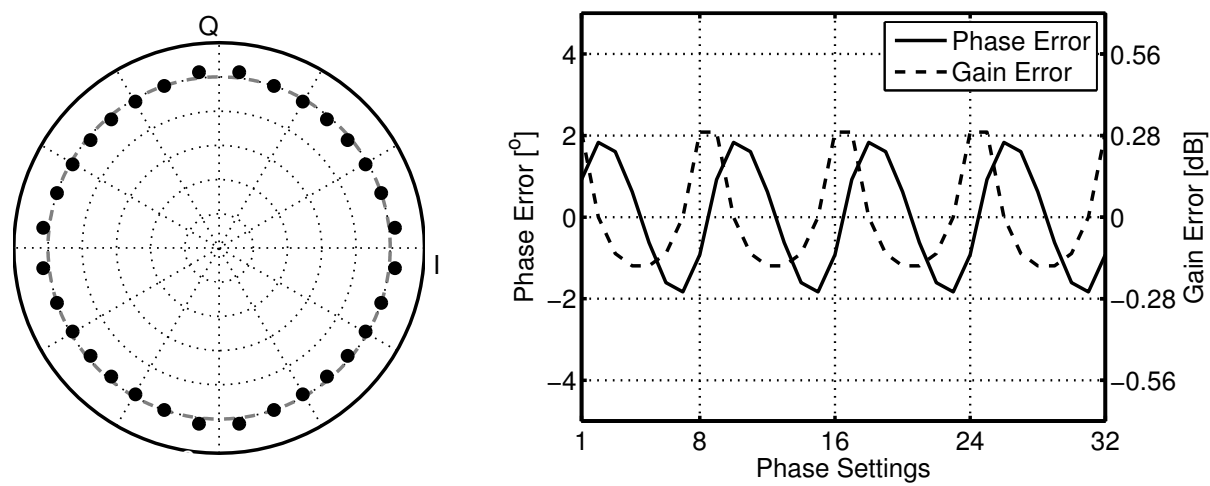

Figure 4.6: Vector modulator phase and gain error for $C_{Z}=3 / 8 C$.

In the circuit of Fig. 4.4b, any capacitance on node $\mathrm{Z}$ will introduce a memory effect. Charge from $\mathrm{X}$ and $\mathrm{Y}$ is retained on $C_{Z}$, resulting in a modification of the transfer function. The effect of this capacitance is shown in Fig. 4.6 for $C_{Z}=3 / 8 \cdot C$. Compared to Fig. 4.5 (with $C_{Z}=0$ ), the gain error increases while the phase error decreases.

The accuracy in phase affects the directions where constructive and destructive summing occurs, i.e. the pointing accuracy of the main beam and nulls. On the other hand, accuracy in gain affects the depth of the nulls. So the capacitance $C_{Z}$ helps to balance the contributions of the phase and gain error to obtain a balanced performance. To find a proper balance, the equivalence between random gain and phase errors can be used as a criterion [30]:

$$
\sigma_{A}=20 \cdot \log _{10}\left(1+\sigma_{\varphi} / 180^{\circ} \cdot \pi\right)
$$



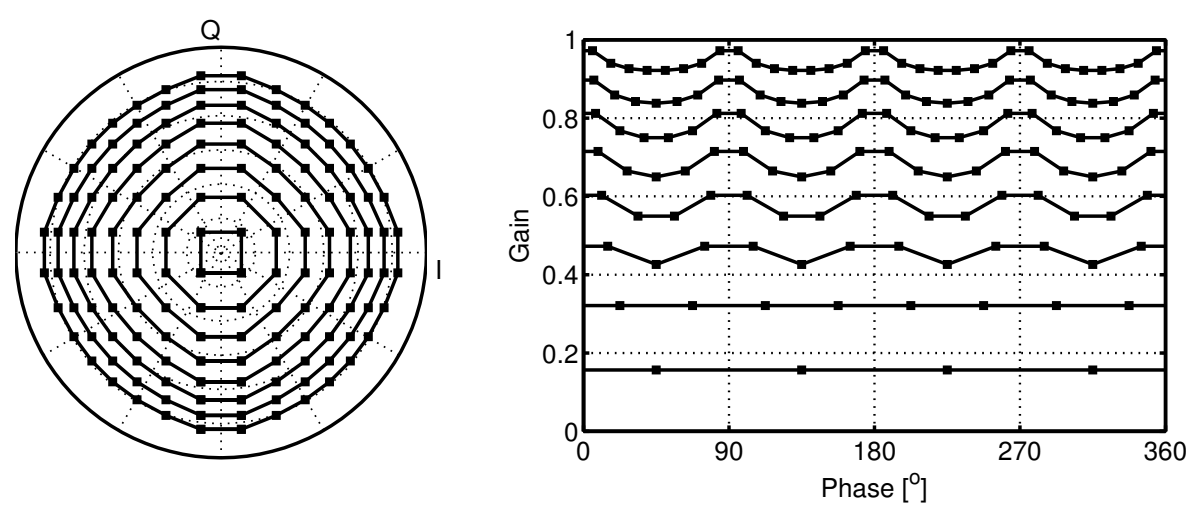

Figure 4.7: Vector modulator constellation with phase and amplitude control.

where $\sigma_{\varphi}$ is the RMS phase error in degrees and $\sigma_{A}$ is the RMS gain error in $\mathrm{dB}$. This equivalence is closely related to the realization that it is the error in distance in the phasor diagram which determines the beamforming performance, so that a distance in amplitude (axial) can be equated to a distance in phase (radial). For the case shown in Fig. 4.6, the RMS phase error is $1.3^{\circ}$ and the RMS gain error is $0.18 \mathrm{~dB}$, resulting in a balanced performance.

\subsubsection{Amplitude Control for Null Steering}

In order to implement the null steering algorithm in section 2.1.5, amplitude control has to be added to the vector modulator. With the proposed circuit in Fig. $4.4 \mathrm{a}$, the amplitude $A$ can be easily controlled by multiplying both $\alpha$ and $\beta$ with the amplitude factors:

$$
\begin{aligned}
& \alpha=A \cdot \varphi \cdot 2 / \pi \\
& \beta=A \cdot(1-\varphi \cdot 2 / \pi) .
\end{aligned}
$$

This is illustrated by the constellation in Fig. 4.7 of the vector modulator with amplitude and gain control, where phase points with equal gain settings are connected by lines. Due to the sine and cosine approximation, the amplitude control is nonuniformly quantized with more gain settings for higher amplitudes. This fits nicely to the requirements for the null steering algorithm, which produces amplitudes close to unity.

It is not trivial to calculate the effect of the non-uniform amplitude quantization on the resulting beam pattern. However, it is possible to estimate the finite null depth due to the uniform phase quantization. In the worst case, a null is needed in the direction of a grating lobe caused by the phase quantization. The height of a 


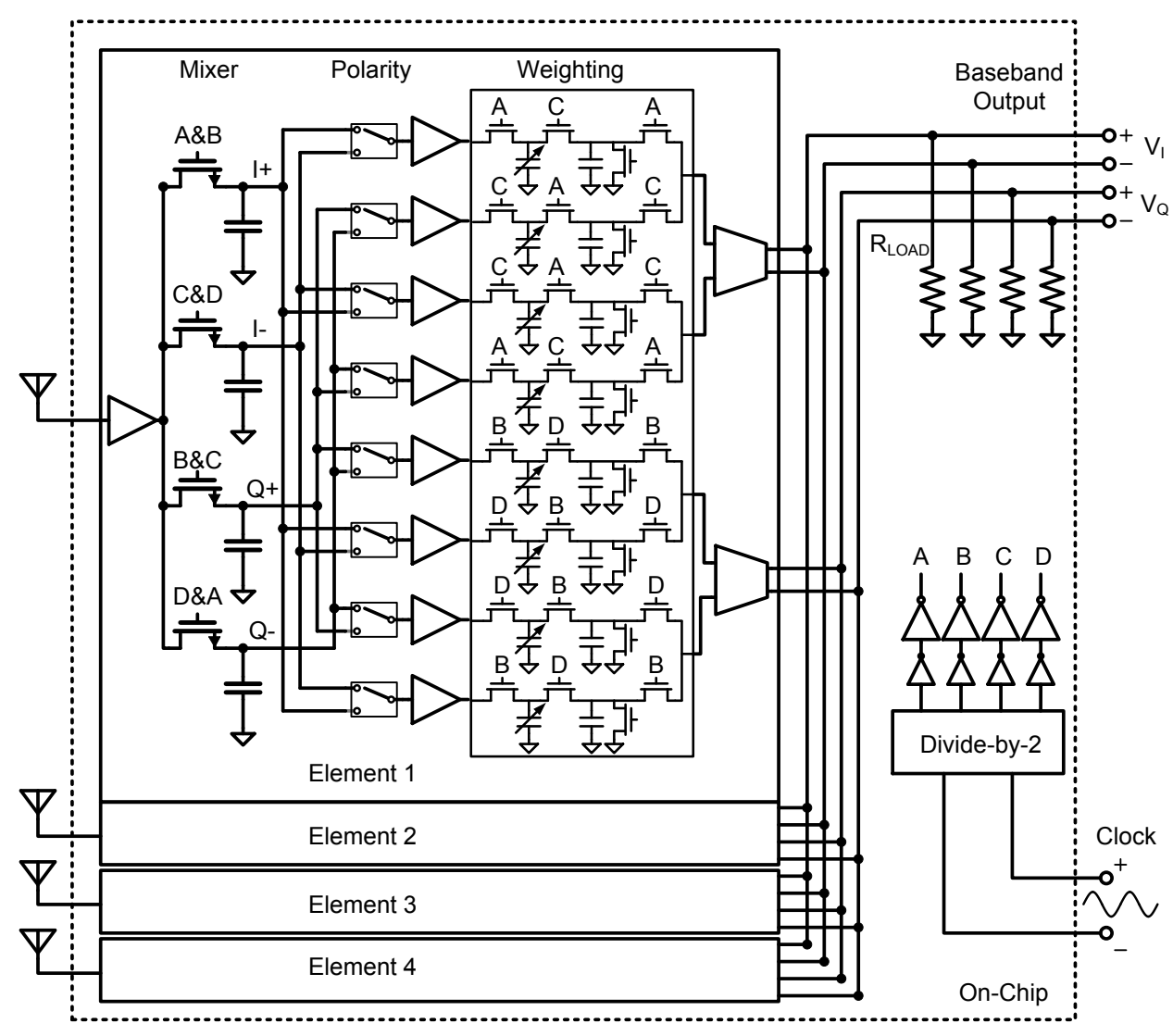

Figure 4.8: 4-element phased-array receiver architecture.

grating lobe below the main beam, $Q L$, can be estimated in $\mathrm{dB}$ as [30]:

$$
Q L \approx 6 M-4
$$

where $\mathrm{M}$ is the number of bits in the phase shifter. This results in a rejection of 26 $\mathrm{dB}$ for 5 bit phase quantization.

\subsection{Implementation in 65-nm CMOS}

To demonstrate the switched-capacitor beamforming, a 4-element phased-array receiver front end, depicted schematically in Fig. 4.8, was implemented. Each element is input matched with a common-gate input stage. Downconversion takes place with an image-reject passive mixer, after which a switched-capacitor vector modulator performs the phase shifting and amplitude scaling. The vector modulator output voltages 


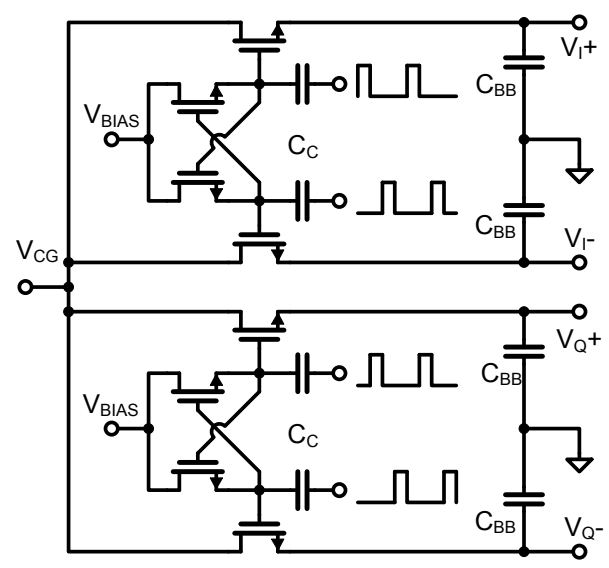

Figure 4.9: Mixer with clock bias.

are converted to current with a transconductance stage and are summed in the current domain. The summed current flows into the common load resistors $R_{\text {load }}$ to provide the IC output voltages. To provide the clock for the mixer and vector modulator, a differential off-chip master clock is divided-by-two to generate a 4 -phase $50 \%$ duty cycle LO.

\subsubsection{Mixer}

To implement the downconversion after the impedance-match input stage, a 4-phase $25 \%$ duty cycle passive mixer is used, as it automatically produces the four inputs required for the vector modulator. In chapter 3 , it was shown that this circuit can provide a high SFDR in the limited supply voltage that is available in 65-nm CMOS technology. In contrast to the mixer-first design, an SE kernel in the mixing region is used, as it is very difficult to route four differential transmission lines towards the RF inputs of the small IC package. Passive baluns are hard to integrate in CMOS, and an on-chip active balun would itself be limiting the second order distortion, invalidating the advantage of the DI kernel.

The mixer is shown in more detail in Fig. 4.9. From the mixer analysis in section 3.1.3, the baseband bandwidth of the SE kernel was found to be:

$$
B W=\frac{D}{2 \pi R C}
$$

In this case, the kernel resistance is formed by the output impedance of the commongate input stage, which is $180 \Omega$. With a baseband capacitor $C_{B B}$ of $3.3 \mathrm{pF}$ and a 


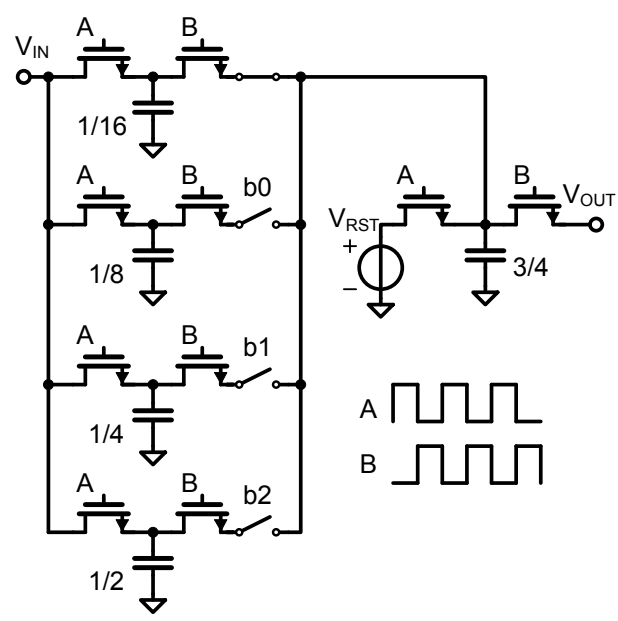

Figure 4.10: Single path in the weighting network.

$25 \%$ duty cycle, this results in about $65 \mathrm{MHz}$ of bandwidth, forming the dominant baseband pole in the system.

The voltage overdrive of the switches is maximized by raising the LO with $V_{\text {bias }}$, the bias voltage on the sources and drains of the mixer switches. This is accomplished by adding cross coupled transistors and coupling capacitors $C_{C}$. When a cross coupled transistor is turned on, the associated coupling capacitor $C_{C}$ is connected to the voltage source on the left plate and to zero voltage on the right plate. This conveniently charges $C_{C}$ to voltage $V_{\text {bias }}$ when the clock is low and the switch is off.

\subsubsection{Vector Modulator}

The architecture block diagram (Fig. 4.8) indicates that the four mixer output phases are offered to four copies of the sine and cosine charge redistribution circuit from Fig. $4.4 \mathrm{~b}$. With the clocking of the three additional copies of the charge redistribution circuit being shifted with respectively a quarter, one-half and three-quarter of the clock period, we can apply the polyphase analysis from section 3.1.4 and see that a differential In-phase and Quadrature output are created. These can be used to perform image rejection. Switches control the polarity of the signal being offered to charge redistribution circuit (selecting the quadrant in the phasor diagram), with voltage buffers added in between to reduce the loading on the mixer capacitors.

Each path in the switched-capacitor weighting network is implemented with the circuit in 4.10. A 3 bit binary capacitor bank is implemented with the three lower, binary weighted parallel paths. The switches are scaled along with the capacitors so that the switch parasitic capacitance scales along to ensure the proper capacitor 


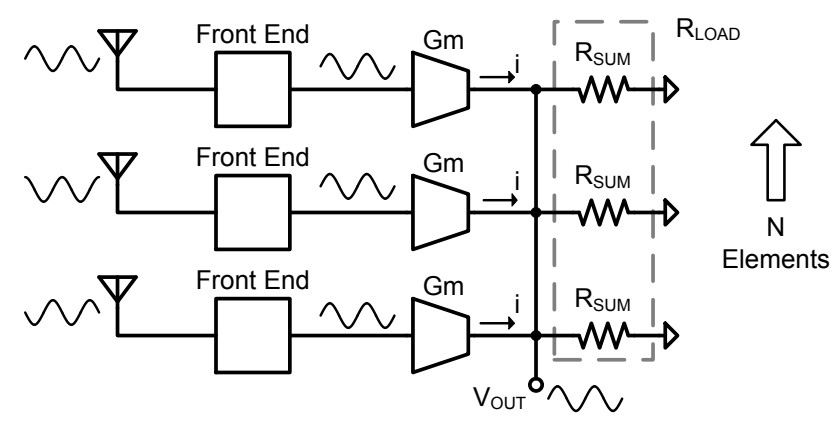

Figure 4.11: Summing in the current domain.

ratios. The upper path is added to provide the correct quantization of $\alpha$ and $\beta$ (always on), which also ensures a path is always present for proper biasing of the next stage. The output capacitor is reset to the bias voltage of the transconductance stage and the transconductance stage itself is scaled to present the correct input capacitance needed for a balanced phase and gain error, as expressed in equation (4.6).

As the mixer and vector modulator run at the same frequency and process at the same sample rate, frequency folding is limited to the already present harmonic upand downmixing of the mixer. In fact, the vector modulator re-uses the RF and channel filter, to perform anti-aliasing and sample reconstruction.

\subsubsection{Element Summing}

After the phase shifting of the signals in the elements, they have to be summed as the last step in beamforming. Fundamentally, this can be done in the voltage domain and in the current domain. Following Kirchhoff's rules, summing in the voltage domain is achieved by stacking voltages on top of each other to form a voltage loop. This is not a practical approach, as the bias voltages are also stacked. In a limited supply voltage, this will impose a strict limit on the number of elements that can be summed.

On the other hand, summing in the current domain is achieved by simply connecting the nodes together. This is illustrated in Fig. 4.11. Considering a single element, the front-end first performs amplification, downconversion and phase shifting. Then, the resulting signal is converted to current with transconductance $G m$ and converted back to voltage with resistor $R_{\text {sum }}$.

For the direction to which the beam is steered, the voltages on the resistors are equal and in-phase, so that connecting the element output nodes together has no net effect. This means that the output voltage has a fixed amplitude, regardless of the number of antenna elements $N$. In fact, increasing $N$ has the effect of lowering the output impedance $R_{\text {load }}$. It is therefore more exact to speak of averaging the signals 


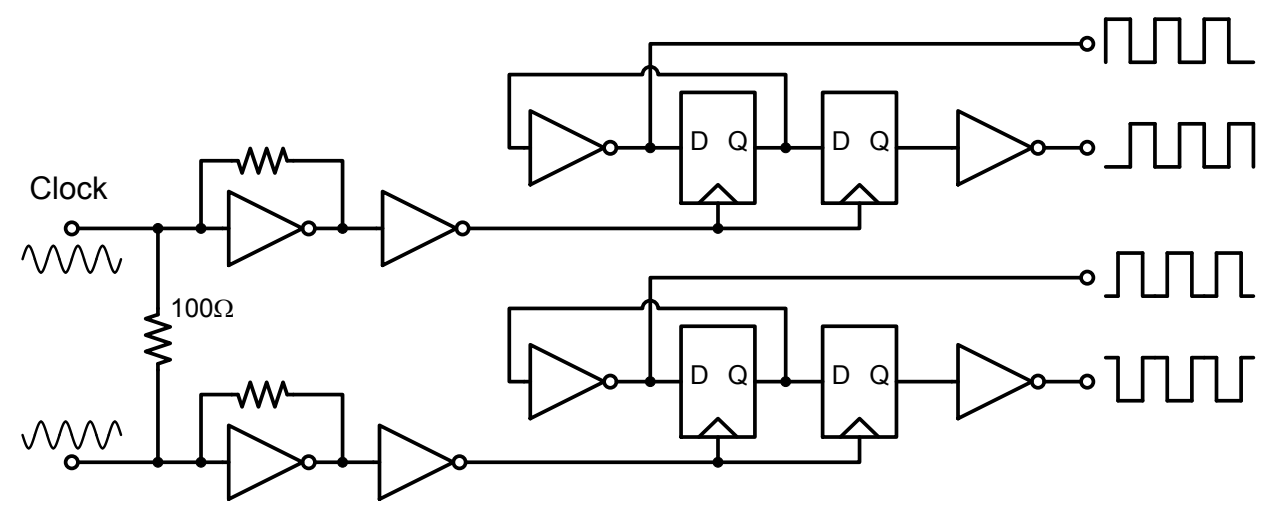

Figure 4.12: Clock divider.

instead of summing the signals. Of course, for directions outside of the main beam, currents will cross over between elements and be (partially) canceled. The noise from the different elements will be averaged out on the summing node, because they are uncorrelated.

One clear advantage of this method is the unlimited scalability in the number of antenna elements. As a phased array is a multiple-input system, the gain is not uniquely defined. Here, the gain is defined as the ratio between the voltage at the input of a element and the output of the array, i.e. it is equal to the gain of a single element and it does not change with the number of elements. For performance metrics like the IP3 and compression point, there is no ambiguity, as these are input related quantities (although we have to take care to excite all input ports at the same time).

For the noise figure of the array, things are different. Fundamentally, there is no noise figure definition for a multiple-input system. It is however possible to start with the noise figure of a single element and indicate the SNR improvement gained by the increased number of antenna elements. The resulting SNR can then be equated to the SNR from a single-antenna system with an equivalent NF. This leads to the negative noise figures (in $\mathrm{dB}$ ) that are sometimes reported for phased arrays and it is important to keep in mind here that the noise figure definition is used outside of its original context. For measuring the noise figure of a single element in an array configuration, the noise setup from [19] can be used.

\subsubsection{Clock generation}

In the mixer-first receiver design, the maximum LO frequency was limited by rise and fall times in the master clock for the divide-by-four clock circuit. By switching to a divide-by-two circuit (Fig. 4.12), the master clock frequency is effectively halved and 


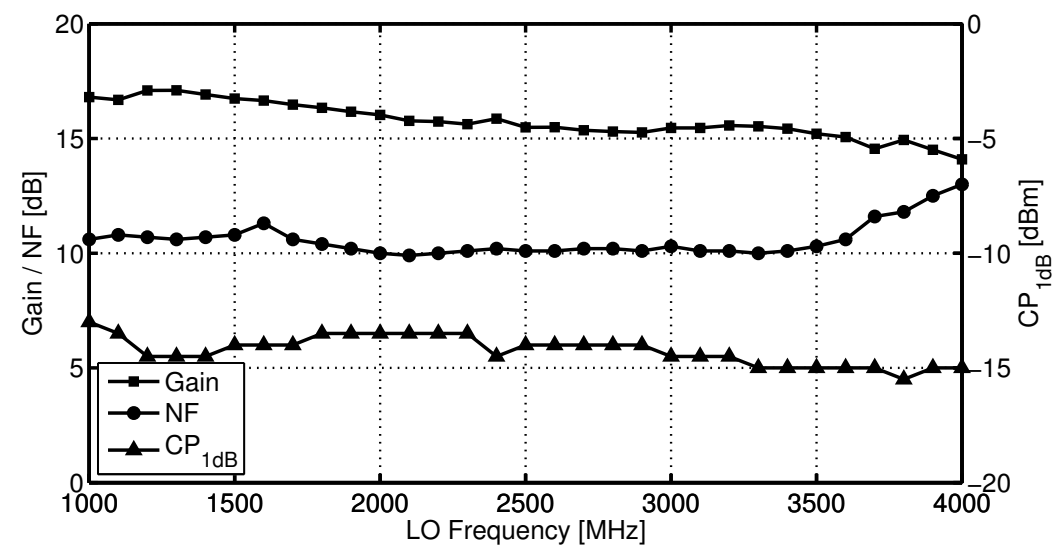

Figure 4.13: Measured receiver element gain, NF and compression point.

$\mathrm{RF}$ bands at higher frequencies can be received by the phased array.

A sinusoidal differential master clock running at twice the LO frequency is first converted to a rectangular signal by dual inverter stages. This drives the dynamic transmission gated flipflops in two divide-by-two loops, one triggering on the rising edge and the other triggering on the falling edge.

The output of the clock divider is distributed along the 4 elements with a binary clock tree, with inverters at each branching node. This effectively doubles the clock power after each clock branch, building up the power from the clock divider up to the power needed to drive all the switches in the elements. Care is taken that in the entire clock tree and clock divider, the capacitive load on the output of a block is twice the capacitance on the input of the block. In digital design terms, this is considered a fan-out of two and it is a compromise between low rise and fall times, and clock driving power.

\subsubsection{Measurements}

The chip is implemented in 65-nm CMOS (die photo in Fig. 4.14), measures $0.9 \mathrm{~mm}$ by $1.2 \mathrm{~mm}$ and has an active area of $0.44 \mathrm{~mm}^{2}$. The beamforming receiver works up to $4 \mathrm{GHz}$ with a $-3 \mathrm{~dB}$ bandwidth after downconversion of $65 \mathrm{MHz}$. With a $1.2 \mathrm{~V}$ supply, the static power consumption is $120 \mathrm{~mW}$, and an additional $188 \mathrm{~mW}$ dynamic power consumption for a $2.5 \mathrm{GHz} \mathrm{LO}$, the latter scaling linearly with LO frequency.

Measurements on a single element are plotted in Fig. 4.13 for LO frequencies between 1 and $4 \mathrm{GHz}$. In the middle of the $\mathrm{RF}$ range at $2.5 \mathrm{GHz} \mathrm{LO}$, a voltage gain of $16 \mathrm{~dB}$ (from the single element input to the differential I output), DSB NF of 10 $\mathrm{dB}$ and in-band input referred compression point of $-14 \mathrm{dBm}$ are measured. In-band 


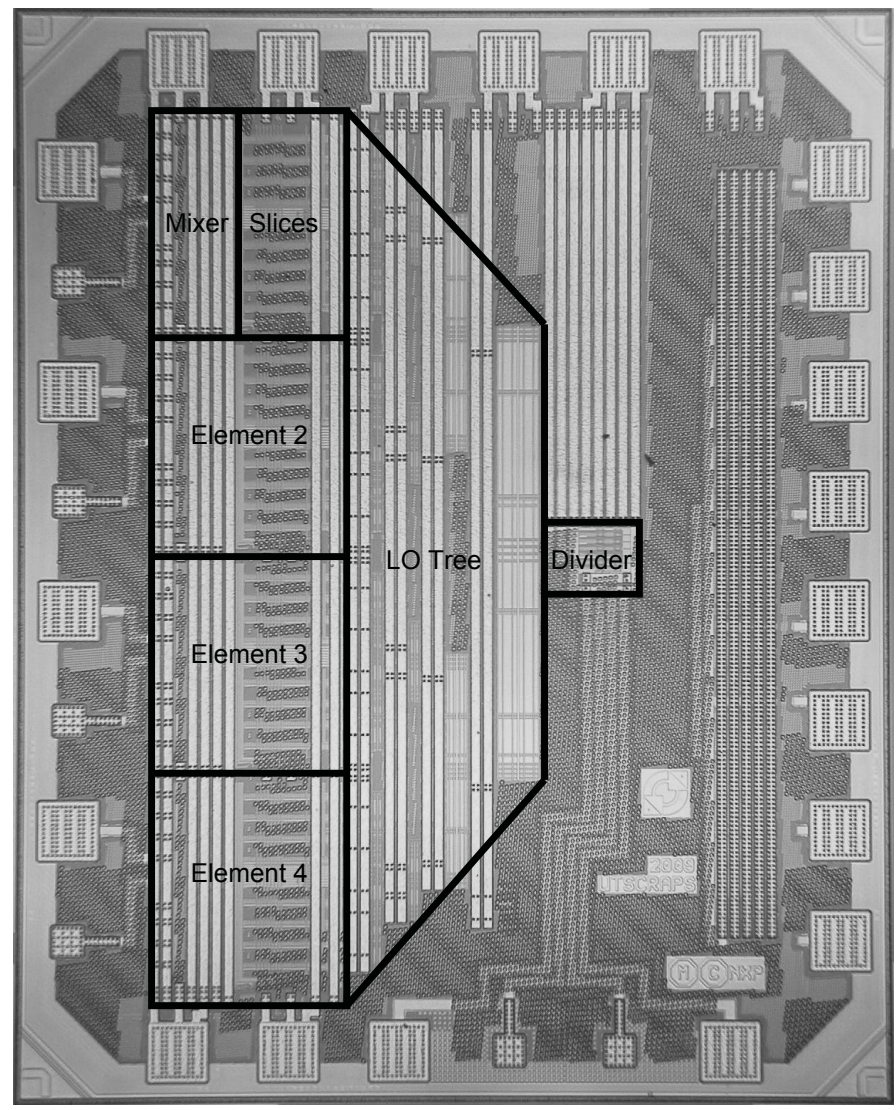

Figure 4.14: Photograph of the $0.9 \mathrm{~mm}$ by $1.2 \mathrm{~mm}$ die.

input referred IP3 is $-1 \mathrm{dBm}$. Above $3.5 \mathrm{GHz}$, the performance deteriorates as the finite rise and fall times of the clock prevent correct functioning of the clock divider.

The measured vector modulator phasor diagram for a single element is shown in Fig. 4.15. Compared to the theoretical constellation in Fig. 4.7, RMS phase error in Fig. 4.15b is as expected. On the other hand, the RMS gain error for the maximum gain setting in Fig. 4.15b has increased somewhat to $0.4 \mathrm{~dB}$. This is probably due to the parasitic layout capacitance on node $\mathrm{Z}$ in Fig. 4.4b and capacitive coupling between source and drain of the switches. The random phase and gain mismatch between vector modulator constellations of different elements are $0.2^{\circ}$ and $0.04 \mathrm{~dB}$ respectively.

To evaluate the actual beamforming patterns of the IC, a setup with four signal generators is used to emulate the incoming phase front from an antenna array and thus directly measure the array factor. The generators have well matched frequencies due to locked reference crystals, but the initial phase differences are unknown. These phase 


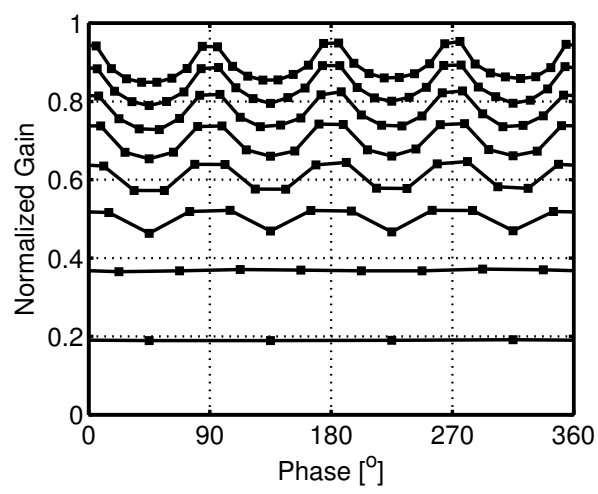

(a)

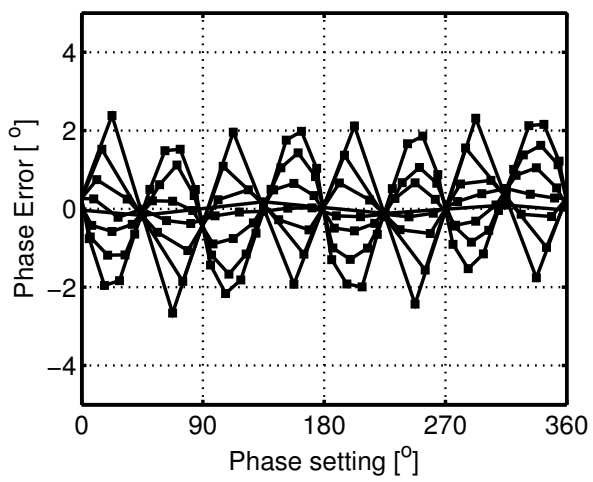

(b)

Figure 4.15: Measured (a) gain-phase and (b) phase-error plot indicating the vector modulator constellation.
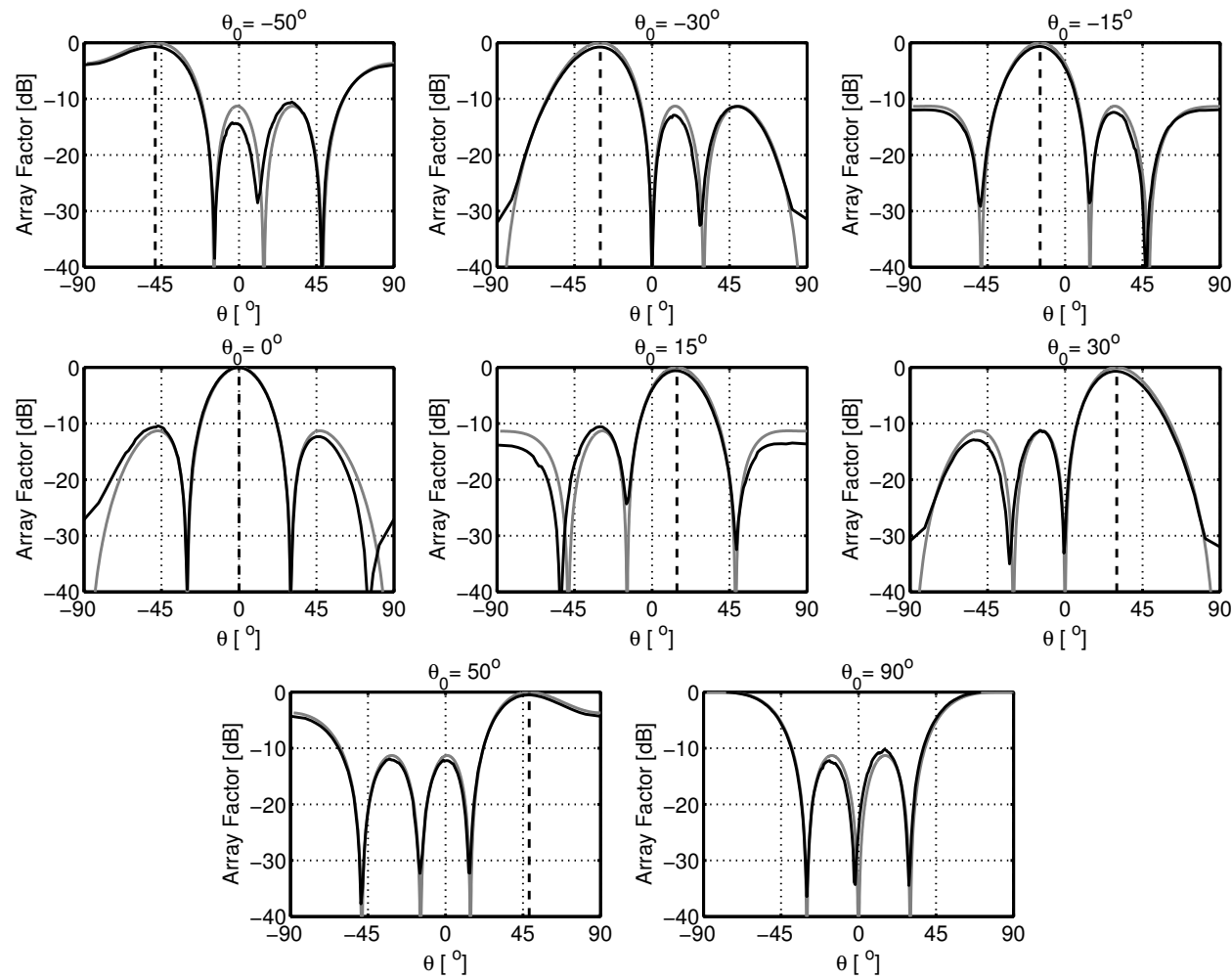

Figure 4.16: Array factors with main beam steered to $-50^{\circ},-30^{\circ},-15^{\circ}, 0^{\circ}, 15^{\circ}, 30^{\circ}$, $50^{\circ}$ and $90^{\circ}$ (ideal in grey, measured in black). 

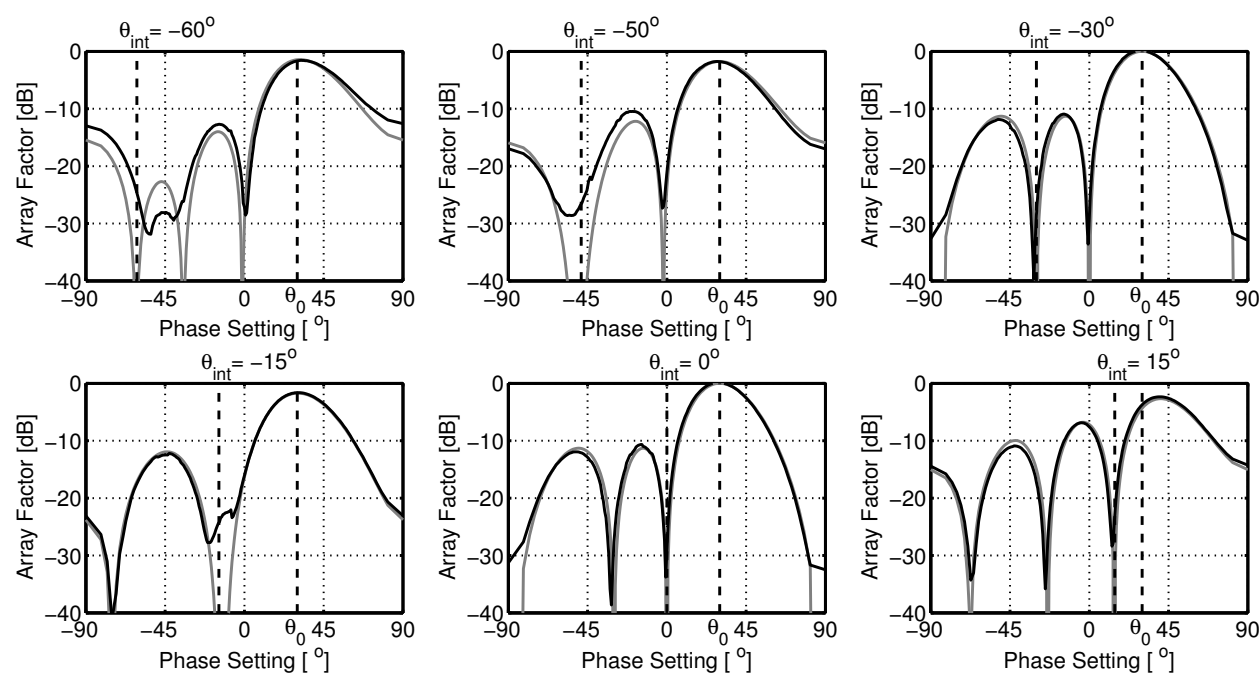

Figure 4.17: Measured array factors with main beam at $30^{\circ}$ and null steered to $-60^{\circ}$, $-50^{\circ},-30^{\circ},-15^{\circ}, 0^{\circ}$ and $15^{\circ}$ (ideal in grey, measured in black).

differences are dynamically calibrated out with a network summing the generator output powers, utilizing destructive summing to detect the phase difference between pairs of generators. A static calibration is done to account for the phase and gain differences introduced by the coupling network and cable up to the PCB connectors, leading to an estimated residual phase and gain errors between the chip inputs of $1^{\circ}$ and $0.2 \mathrm{~dB}$ respectively. In the setup, PCB micro strip coupling introduces -20 $\mathrm{dB}$ coupling between adjacent RF inputs, a situation which is likely to occur in a practical antenna array.

First, a measurement of the beam steering is performed with this setup. Fig. 4.16 plots the array factors for several beam steering settings, which correspond closely to the ideal array factors. Sources of error include amplitude and phase mismatches between elements, systematic errors due to the sine and cosine approximation, and coupling between elements.

Furthermore, the nulling capability through amplitude control is demonstrated by applying the nulling algorithm from section 2.1 .5 to produce vector modulator settings that give an array factor with a main beam at $30^{\circ}$ and a null between $-60^{\circ}$ and $15^{\circ}$. The measured patterns are plotted in Fig. 4.17. Even though the null is steered up to only $15^{\circ}$ from the main beam, a spatial rejection of more than $20 \mathrm{~dB}$ is reached for this 4-element beamformer. In light of the additional errors introduced by the measurement setup, this is not far off from the initial estimate of $26 \mathrm{~dB}$ of spatial interferer rejection.

The measured performance is summarized in Table 4.1. Approximating the sine 
Table 4.1: Performance Summary

\begin{tabular}{|l|c||l|c|}
\hline Phased Array: & Single Element: & @ 2.5 GHz RF \\
\hline Technology & $65-\mathrm{nm}$ CMOS & Gain & $16 \mathrm{~dB}$ \\
\hline Active area & $0.44 \mathrm{~mm}^{2}$ & Noise figure & $10 \mathrm{~dB} \mathrm{DSB}$ b \\
\hline RF frequency band & $1-4 \mathrm{GHz}$ & $\begin{array}{l}1 \mathrm{~dB} \text { compression } \\
\text { point }\end{array}$ & $-14 \mathrm{dBm}$ \\
\hline $\begin{array}{l}\text { Power } \\
\text { consumption }\end{array}$ & $308 \mathrm{~mW} @ 1.2 \mathrm{~V}^{\mathrm{a}}$ & In-band IIP3 & $-1 \mathrm{dBm}$ \\
\hline Array directivity & $6 \mathrm{~dB}$ & In-band IIP2 & $40 \mathrm{dBm}$ \\
\hline $\begin{array}{l}\text { Element phase } \\
\text { mismatch }\end{array}$ & $0.2^{\circ} \mathrm{RMS}$ & Phase control & $5 \mathrm{bit}$ \\
\hline $\begin{array}{l}\text { Element amplitude } \\
\text { mismatch }\end{array}$ & $0.04 \mathrm{~dB} \mathrm{RMS}$ & Phase error & $1.4^{\circ} \mathrm{RMS}$ \\
\hline $\begin{array}{l}\text { Main beam-to-null } \\
\text { ratio }\end{array}$ & $>20 \mathrm{~dB}$ & Amplitude error & $0.4 \mathrm{~dB}$ RMS \\
\hline
\end{tabular}

a $120 \mathrm{~mW}$ static, $188 \mathrm{~mW}$ dynamic @ $2.5 \mathrm{GHz} \mathrm{RF}$

b Additional 6dB SNR improvement for full array

and cosine in the vector modulator has produced low systematic gain and phase errors. Moreover, as the phase shift depends on the ratio of capacitors, the constellation points are well controlled and matching between elements is good.

\subsection{Conclusions}

In this chapter a switched-capacitor vector modulator has been presented and used in a single-chip 4-element phased-array receiver. A rational approximation of the sine and cosine was proposed in order to generate the non-uniformly quantized weights needed for uniform phase steps. This approximate-sine weighting is mapped on a charge-redistribution circuit and results in all vector modulator settings being used for effective phase shifts. As the phase shift depends on the ratio between capacitors, it is very well controlled.

The 65 -nm CMOS implementation with 5 bit phase control and 3 bit amplitude control in the vector modulator achieves an RMS systematic phase and gain error of $1.4^{\circ}$ and $0.4 \mathrm{~dB}$ respectively. Applications in crowded frequency bands, like the 2.4 $\mathrm{GHz}$ Industrial Scientific Medical (ISM) band, are enabled by the high $-1 \mathrm{dBm}$ IIP3. Moreover, a spatial interference rejection $>20 \mathrm{~dB}$ through nulling was demonstrated, to lower the linearity requirements on the baseband receiver part. 


\section{Chapter 5}

\section{All-Passive}

\section{Switched-Capacitor Phased}

\section{Array}

In chapter 3, it was demonstrated that a mixer-first architecture can have a large spurious-free dynamic range. Instrumental for this approach was the use of passive $25 \%$ duty cycle mixers. In the frequency domain analysis it was concluded that for low noise, mixing region switched-RC loops can be used in the mixer design. Even so, the linearity of the implemented prototype was not limited by the mixer, but by the active baseband stages.

On the other hand, chapter 4 introduced sampling region switched-RC loops for the phase shifter design. In this case, a time domain approach proved useful in finding the circuit transfer function and a very stable phase shifter performance was demonstrated. In the resulting prototype, it were also the active stages that limited the linearity performance.

The question arises if the mixer-first architecture can be combined with the sampling region phase shifter, without the use of intervening active stages, as to achieve a higher linearity. This fits naturally to the strengths of CMOS technology, as such a design would only use switches and capacitors. Of course, active stages have to be involved at some point, but can be inserted at places where they hurt the least.

With that in mind, the phased-array system design in Fig. 5.1 is proposed. The active CMOS baseband circuits are placed after the beamforming summing node, so that strong interferers can be spatially filtered. These interferers are still present in the beamforming circuits, which are passive and provide a $50 \Omega$ interface to the RF ports. RF band filters are necessary to filter out signals at the LO harmonics, 


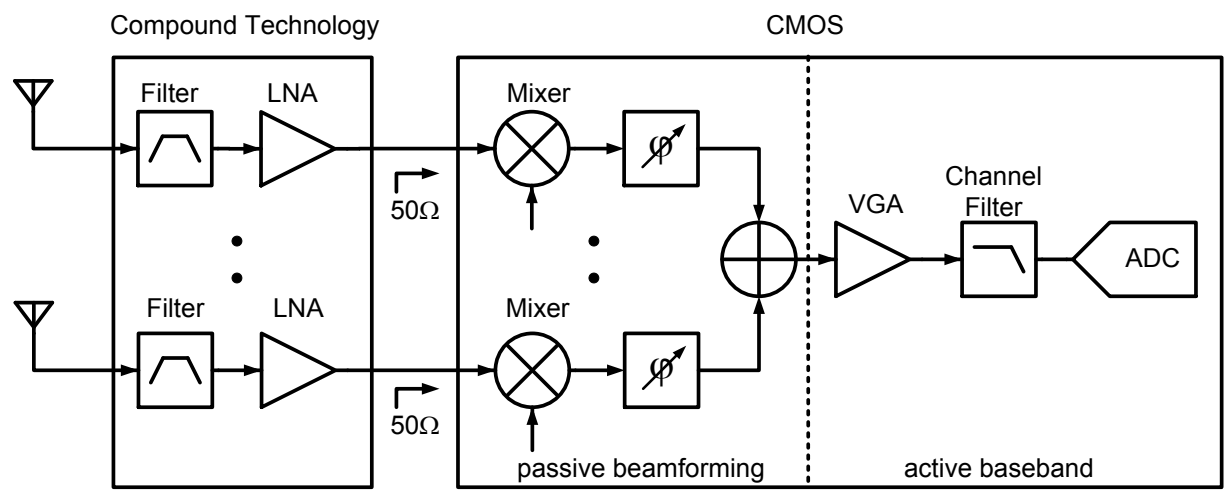

Figure 5.1: Proposed high-linearity beamforming architecture.

while LNAs in (relatively) high voltage IC technologies can be added for low noise applications without compromising the linearity.

For the CMOS passive beamforming part of the architecture, which provides the phase shifting, element summing and downconversion functionality, the absence of active stages influences the design in two ways. First, the impedance matching at the input ports, normally provided by the LNA, has to be implemented by other means. Secondly, consider that the passive mixer and switched-capacitor phase shifter can not be directly connected together without seriously affecting each others functionality. This is due to the loading of one stage to another, as these circuits operate on careful charge balancing. The previous analysis results from chapter 3 and 4 do not take these effects into account and are not sufficient to perform the design.

Therefore, this chapter first introduces a method for designing larger systems with a mixture of mixing and sampling region switched-RC loops. This design methodology is then used to design a fully-passive coupled switched-capacitor circuits that perform the required functionality for the beamforming architecture.

\subsection{Coupling of Switched-RC Loops}

In the analysis of switched-RC loop coupling, it is instructive to begin by reviewing the operation of switched-RC loops in the time domain. To this end, the step response of the RC network in Fig. 5.2 is analyzed. The switch closes at $t=0$ and opens again at $t=T_{O N}$, simulating one cycle in a switched-RC loop. Initially the capacitor is charged to voltage $V_{0}$, the final value of the voltage at the previous cycle.

As was discussed in the frequency domain analysis of Chapter 3, it depends on the switch-on time $T_{O N}$ what the behavior of this circuit is in a periodically switched network. If the switch opens much later than twice the $R C$ time constant $(\Gamma \gg 2)$, 

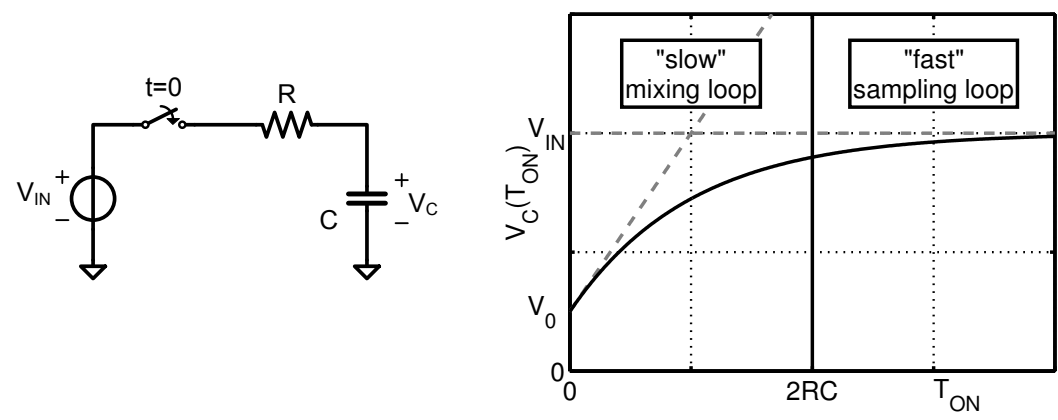

Figure 5.2: RC model of switching loop with time response.

the capacitor voltage has time to settle to the input voltage. The kernel operates in the sampling region and is defined as being a fast loop. The charge $Q$ that is transferred from the source to the capacitor is just the capacitance times the voltage difference:

$$
Q\left(T_{O N}>>2 R C\right) \approx\left(V_{I N}-V_{0}\right) \cdot C
$$

So, the amount of transferred charge does not depend on the resistance or switch-on time, as long the switch is closed long enough to ensure settling.

On the other hand, if the switch opens much earlier than twice the $R C$ time constant $(\Gamma \ll 2)$, the capacitor voltage has not had enough time to settle to the input voltage. The kernel operates in the mixing region and is defined as being a slow loop. It can be seen from the time response that the capacitor voltage rises approximately linear, with the asymptote proportional to the resistance in the loop. In this case, the charge transferred to the capacitor is:

$$
Q\left(T_{O N}<<2 R C\right) \approx\left(V_{I N}-V_{0}\right) \cdot \frac{T_{O N}}{R}
$$

Now, the transferred charge is proportional to the switch-on time and the resistance, while being independent of the capacitance.

This difference in behavior in the time domain is consistent with the spot noise of an RC kernel in Fig. 3.14 being proportional to resistance for slow loops and proportional to capacitance for fast loops. Moreover, these insights will help in making the correct approximations in deriving the coupled behavior of fast and slow loops ${ }^{1}$.

\footnotetext{
${ }^{1}$ The question might arise: why does the boundary between the two regions turn out to be twice the RC time constant? In the step response in Fig. 5.2, it is seen that the step response starts to fall on top of the asymptotes for the sampling and mixing region at the respective right and left border of the figure. On the time axis, twice the RC time constant is halfway between those points.
} 


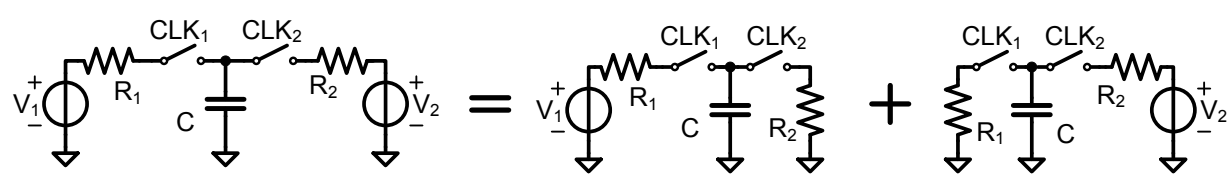

Figure 5.3: Two slow loops $(\Gamma \ll 2)$ acting on the same capacitor.
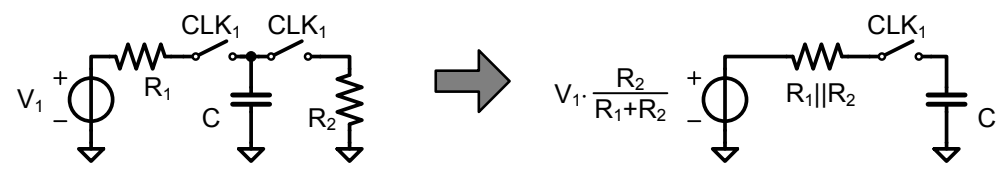

Figure 5.4: Transformation into equivalent single loop.

\subsubsection{Mixing Region Loops}

It was derived in section 3.1.3 that the harmonic transfer function of a single slow loop is equal to:

$$
H_{n, S E}\left(f_{o}\right) \approx \frac{\operatorname{sinc}(D n)}{1+j 2 \pi \frac{R C}{D} f_{o}} \cdot e^{-j 2 \pi\left(t_{0}+\frac{D}{2}\right) n}, \quad \Gamma \ll 2 .
$$

where $D$ is the clock duty cycle, $R$ is the total resistance in the loop and $C$ is the load capacitance. In this equation, the phase shift due to the starting time $t_{0}$ of the clock pulse is included.

Now, consider the situation in Fig. 5.3, where two voltage sources with individual resistors and switches act on the same capacitor. The superposition principle can be applied to express the total response as the sum of the response of the individual voltage sources, as is illustrated in the figure. The total HTF is therefore the sum of the individual HTFs of the two sources:

$$
H_{n}\left(f_{0}\right)=H_{n, 1}\left(f_{o}\right)+H_{n, 2}\left(f_{o}\right)
$$

Analysis of $H_{n, 1}\left(f_{o}\right)$ and $H_{n, 2}\left(f_{o}\right)$ is practically identical and the focus is now on the first.

In the case where the two switches have the same duty cycle and starting moment, the switches are effectively on at exactly the same time and LTI network analysis can be used to transform into a single loop. Resistors $R_{1}$ and $R_{2}$ form a voltage divider, adding a scaling factor in (5.3). The capacitor now sees the parallel combination of $R_{1}$ and $R_{2}$, which constitutes the effective $R$ of the loop. This transformation into a single loop is illustrated in Fig. 5.4.

What if the clocking of the second loop starts at a later time? As Fig. 3.9b illustrated, the voltage on the capacitor in a slow loop changes only a little at a time 
and can be considered quasi-static during each cycle. For the charge dissipation in the second loop, (5.2) can now be used with zero $V_{I N}$ and quasi-static $V_{0}$. Therefore, it does not matter at which time in the cycle the second loop dumps charge to ground and the transformation in Fig. 5.4 is valid for all starting moments.

Moreover, from (5.2) it can be learned that the amount of dissipated charge is proportional to the switch-on time of the second loop. Similarly, the amount of charge transferred from the voltage source is proportional to the switch-on time of the first loop. This observation leads to the realization that the voltage division is according to the effective loop resistances $R_{1} / D_{1}$ and $R_{2} / D_{2}$, where $D_{1}$ and $D_{2}$ are the duty cycles of the respective loops. The effective resistance of the first loop has been in the single loop HTF all along, as the factor $R / D$ in (5.3), and the HTF for two loops includes the parallel combination of the two effective resistances:

$$
H_{n, 1}=\frac{\frac{R_{1}}{D_{1}} \| \frac{R_{2}}{D_{2}}}{\frac{R_{1}}{D_{1}}} \cdot \frac{\operatorname{sinc}(D n)}{1+j 2 \pi\left(\frac{R_{1}}{D_{1}} \| \frac{R_{2}}{D_{2}}\right) C \cdot f_{o}} \cdot e^{-j 2 \pi\left(t_{1}+\frac{D_{1}}{2}\right) n}
$$

Equivalently it can be derived that:

$$
H_{n, 2}=\frac{\frac{R_{1}}{D_{1}} \| \frac{R_{2}}{D_{2}}}{\frac{R_{2}}{D_{2}}} \cdot \frac{\operatorname{sinc}(D n)}{1+j 2 \pi\left(\frac{R_{1}}{D_{1}} \| \frac{R_{2}}{D_{2}}\right) C \cdot f_{o}} \cdot e^{-j 2 \pi\left(t_{2}+\frac{D_{2}}{2}\right) n}
$$

If more than two loops are acting on a capacitor, the result will be additional terms in the superposition sum and more terms in the parallel combination of effective resistances.

Consider the situation where the two loops have equal duty cycle and resistance, with $V_{1}=-V_{2}$ and the second clock shifted by half a period $\left(t_{2}=t_{1}+T_{S} / 2\right)$. According to (5.5) the parallel combination of the resistances results in half the bandwidth and a halving of the transfer function from each source to the capacitor. Moreover, the half period time shift between the clocks gives a phase shift in the contribution to the second source, so that second harmonics are canceled in the summation. The summed HTF can now be expressed as:

$$
H_{n}\left(f_{o}\right)=\left(1-e^{-j \pi n}\right) \cdot \frac{\operatorname{sinc}(D n)}{1+j 2 \pi \frac{R C}{2 D} f_{o}} e^{-j \pi D n}
$$

which is the same result as derived for the DI kernel (3.17) in chapter 3, giving confidence in the validity of this analysis.

\subsubsection{Input-Matched Passive Mixer}

An interesting application of the previous analysis results lies in the circuit on the left in Fig. 5.5, which has $25 \%$ duty cycle $D$ clocks. There is a primary loop with voltage 

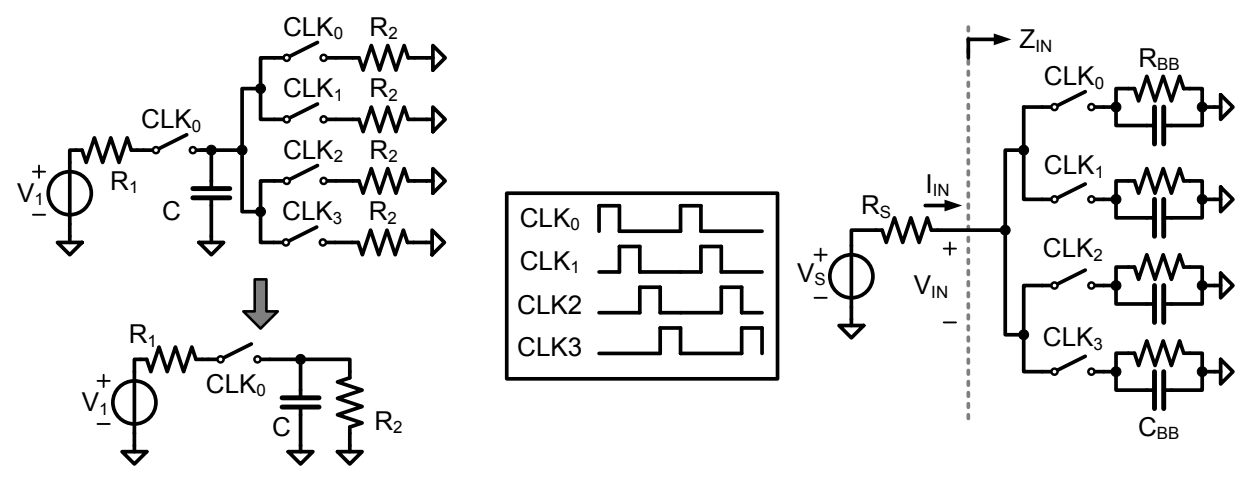

Figure 5.5: Input matched passive mixer.

source $V_{1}$ and four secondary loops to ground. Conceptually, the secondary loops can be replaced by a single resistor to ground without switching, as the $R_{2}$ resistors have no memory.

Whether this circuit is analyzed as having four secondary loops with $25 \%$ duty cycle or as a single secondary loop with $100 \%$ duty cycle gives no difference in the results by application of (5.5). The voltage division is equal to $\left(R_{1} \| R_{2} D\right) / R_{1}$ and the effective total loop resistance is equal to $R_{1} / D \| R_{2}$. So, the HTFs of the circuit can be expressed as:

$$
H_{n}\left(f_{o}\right)=\frac{R_{1} \| R_{2} D}{R_{1}} \cdot \frac{\operatorname{sinc}(D n)}{1+j 2 \pi\left(R_{1} / D \| R_{2}\right) C f_{o}} .
$$

In a four-path configuration, this circuit has been studied to provide impedance matching at the input port [58], as shown on the right in Fig. 5.5. With the transfer functions to the output nodes known from (5.8), the transfer function to the input node with voltage $V_{I N}$ can be calculated.

For ideal switches, the input node is alternately connected to each of the output nodes for a quarter of the clock period. In the time domain therefore, the voltage on the input node is the sum of the voltages on the output nodes times the respective clock waveform. The different timing for the output nodes results in a phase shift in the frequency domain, as was derived in the polyphase analysis in section 3.1.4. Together with the realisation that a multiplication with a rectangular clock wave in the time domain is a convolution with a sinc function in the frequency domain, the transfer function to the input node can be written as:

$$
H_{i n}(f)=\frac{V_{I N}\left(f_{i}\right)}{V_{S}\left(f_{i}\right)}=\sum_{i=0}^{3}\left[e^{-j 2 \pi \frac{i}{4}} \cdot \sum_{k}\left(\operatorname{sinc}(D k) \cdot H_{n}\left(f_{i}-k f_{s}\right)\right)\right] .
$$

For impedance matching, we are interested in frequencies around $f_{s}$. Equation (5.9) indicates that after downconversion to the output node with frequency shift 

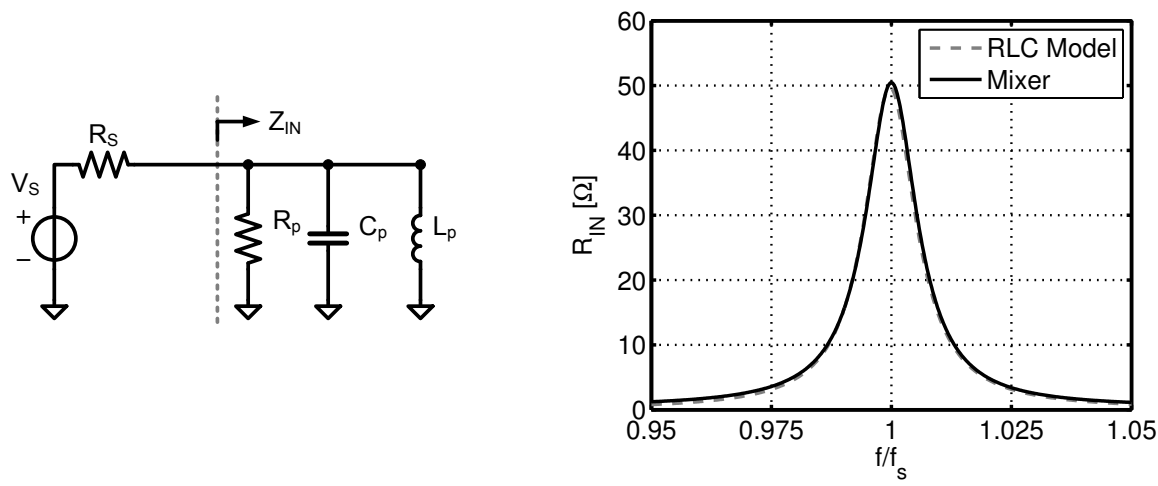

Figure 5.6: RLC input impedance model with its simulation result compared to the mixer.

$n f_{s}$, the signal is upconverted to the input node with frequency shift $k f_{s}$. As the output node is lowpass filtered, it follows that around $f_{i}=f_{s}$, the major contribution to $H_{\text {in }}(f)$ comes for $n=-1$ and $k=1$, corresponding to a downconversion and upconversion by $f_{s}$.

Substituting equation (5.8) into (5.9) and applying $n=-1$ and $k=1$ gives the transfer function to the input around $f_{s}$ :

$$
H_{i n}\left(f_{i}\right)=\frac{V_{I N}\left(f_{i}\right)}{V_{S}\left(f_{i}\right)}=\frac{R_{S} \| R_{B B} D}{R_{S}} \cdot \frac{\operatorname{sinc}^{2}(D)}{1+j 2 \pi\left(R_{S} / D \| R_{B B}\right) C\left(f_{i}-f_{s}\right)} .
$$

It is revealed that the lowpass filtering towards the output (5.8) is transformed into bandpass filtering at the input (5.12), by shifting the double-sided lowpass filtering response upto frequency $f_{s}$. This method of accomplishing bandpass filtering was already described in the 1960s for N-path filters [59].

Using this transfer function, the input impedance is calculated as:

$$
\begin{gathered}
Z_{I N}\left(f_{i}\right)=\frac{V_{I N}\left(f_{i}\right)}{I_{I N}\left(f_{i}\right)}=\frac{R_{S} \cdot V_{I N}\left(f_{i}\right)}{V_{S}\left(f_{i}\right)-V_{I N}\left(f_{i}\right)}=\frac{R_{p}}{1+j 4 \pi\left(f_{i}-f_{s}\right) R_{p} C_{p}} \\
R_{p}=R_{S} \cdot \frac{\operatorname{sinc}^{2}(1 / 4)}{1-\operatorname{sinc}^{2}(1 / 4)} \| R_{B B} \cdot \frac{\operatorname{sinc}^{2}(1 / 4)}{4} .
\end{gathered}
$$

This input impedance can be approximated by a $2^{\text {nd }}$ order resonant circuit with resonance frequency $f_{s}$, by applying a series expansion [60][61]. This results in he simple parallel RLC model on the left side in Fig. 5.6, with component values $R_{p}$ and:

$$
C_{p}=\frac{2 C_{B B}}{\operatorname{sinc}^{2}(1 / 4)}, \quad L_{p}=\frac{1}{4 \pi^{2} f_{s}^{2} C_{p}} .
$$




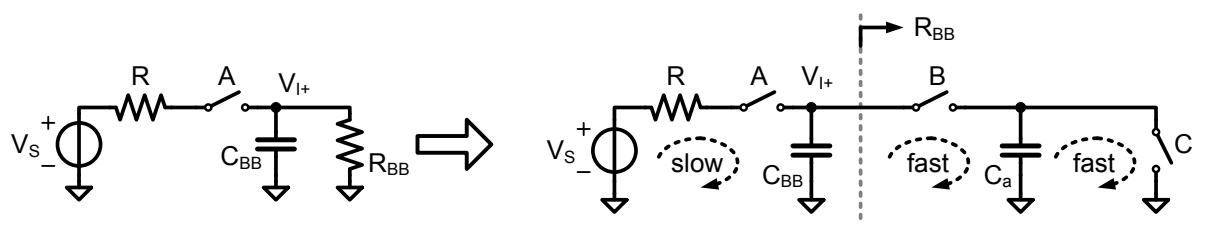

Figure 5.7: Replacing a resistor with fast switched-RC loops.

A simulation comparison of the input impedance of the full mixer and the RLC equivalent input model is plotted on the left side of Fig. 5.6, confirming the validity of the model.

Under input impedance matching conditions, the transfer function to the input node (5.9) has to be equal to $1 / 2$. It follows therefore that for $25 \%$ duty cycle, input matching is achieved when:

$$
\operatorname{sinc}(1 / 4)^{2} \cdot \frac{R_{B B}}{4 R_{S}+R_{B B}}=\frac{1}{2}
$$

which can be rewritten to give:

$$
R_{B B}=R_{s} \cdot \frac{4}{4 \operatorname{sinc}^{2}(1 / 4)-1} \approx 6.5 R_{S} .
$$

In this approach the analysis was performed by first determining the transfer function to all nodes and then deriving the respective impedances. The achieved results are identical to the ones reached by Andrews [62] and Mirzaei [63], who adopted the reverse method by first calculating the transformation of impedances from node to node, and then deriving the resulting node transfer functions.

\subsubsection{Sampling Region Loops and Resistor Simulation}

As was shown, in order to provide input matching a real impedance has to be presented as a load at the passive mixer outputs. Instead of using a resistor, a switched capacitor network can be used, as is shown in Fig. 5.7. It is essential to realize that $C_{a}$ is much smaller than $C_{B B}$. As such, the current loop associated with switch $A$ has no time to settle and operates in the mixing region as defined in section 3.1. On the other hand, the current loops associated with switches $B$ and $C$ have enough time to settle and operate in the sampling region. Note that for switch $B$, capacitor $C_{B B}$ acts as the voltage source as it is much bigger than $C_{a}$ and remains charged. Similarly, for the loop through switch $C$, the voltage on $C_{a}$ itself acts as the voltage source in the loop.

For the right-hand circuit in Fig. 5.7, this means that in each cycle, $C_{a}$ is charged to voltage $V_{I+}$ and later on completely discharged through switch $C$. In a sense, this means that a fixed packet of charge is dissipated for each clock cycle. With a 

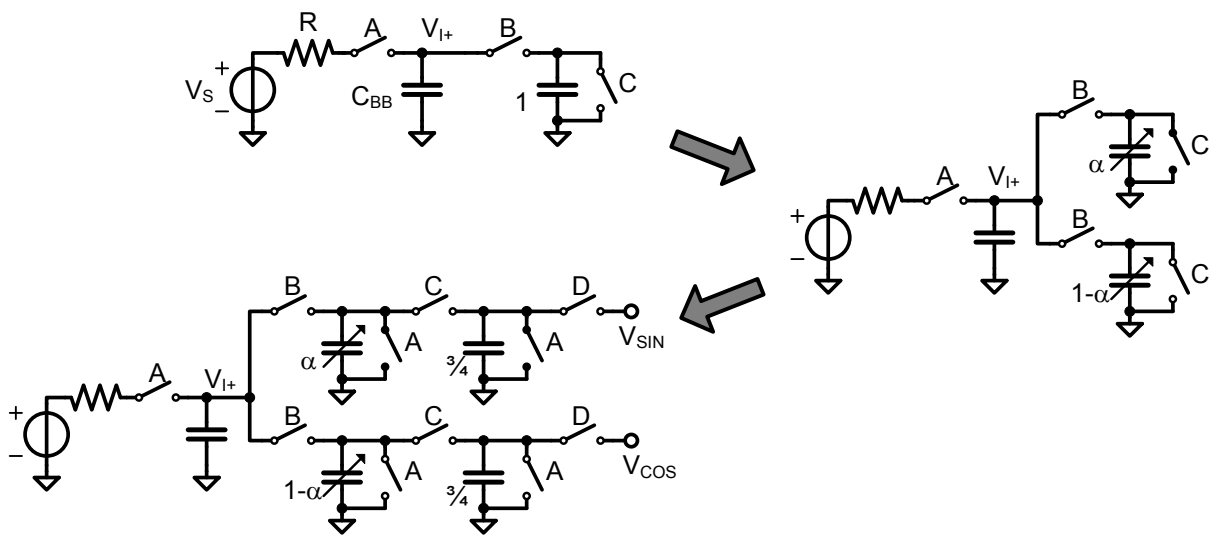

Figure 5.8: Sine/cosine approximation.

clock frequency of $f_{s}$, this means that the average current for quasi-static $V_{I N}$ can be expressed as:

$$
\overline{I_{I N}}=V_{I N} \cdot C_{a} \cdot f_{s}
$$

And therefore, the low frequency impedance of the switched capacitor network can be expressed as:

$$
R_{B B}=\frac{1}{C_{a} \cdot f_{s}}
$$

In a way, $R_{B B}$ is the interface resistance between the slow loops and fast loops in Fig. 5.7. It models the load of the fast loops on the slow loops, resulting in impedance matching at the RF input as calculated in (5.15).

\subsubsection{Phase Shifting}

By replacing baseband resistor $R_{B B}$ with sample-and-reset capacitor $C_{a}$, there is a packet of charge available on $C_{a}$. As this charge is dissipated to ground, it can be manipulated as long as it is completely dissipated before the next clock cycle. Fig. 5.8 demonstrates how the charge redistribution principle from section 4.1.2 can be applied to the charge packet to provide a sine and cosine approximation.

First of all, the upper left of the figure depicts the circuit from Fig. 5.7, but with $C_{a}$ scaled as a unit capacitor. Mixer capacitor $C_{B B}$ is much larger than one, to ensure a slow mixer loop together with the source resistance. Capacitor $C_{a}$ can conceptually be split into two parts according with variable fraction $\alpha$, without affecting the input impedance.

Now, the two capacitors carry charge $V_{I+} \cdot \alpha$ and $V_{I+} \cdot(1-\alpha)$ respectively. In the next step, the charge on these capacitors can be redistributed with fixed $3 / 4$ sized 


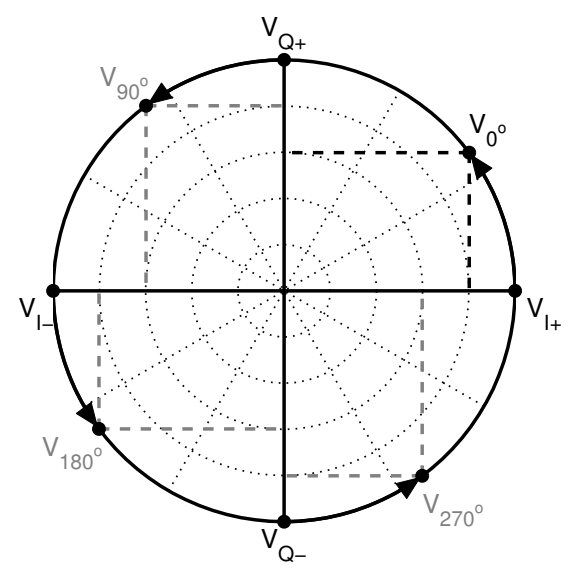

(a)

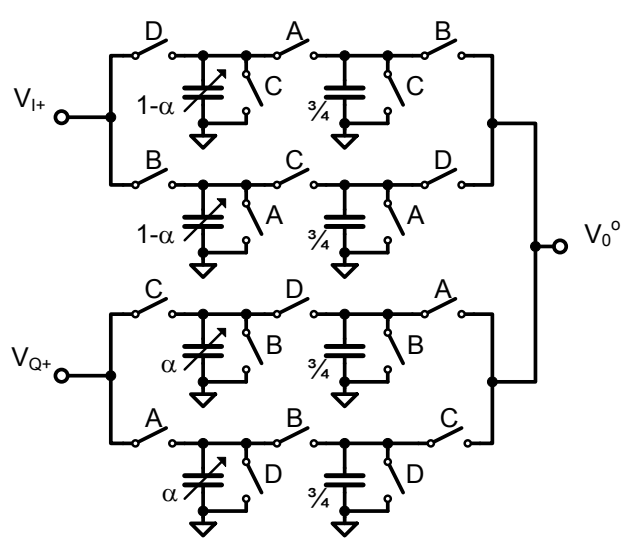

(b)

Figure 5.9: Vector modulator (a) phasor diagram and (b) wiring schematic.

capacitors to form the two output voltages:

$$
\begin{gathered}
V_{C O S}=V_{I+} \cdot \frac{\alpha}{\alpha+3 / 4} \approx V_{I+} \cdot \frac{4}{7} \cos \left(\alpha \cdot 90^{\circ}\right) \\
V_{S I N}=V_{I+} \cdot \frac{(1-\alpha)}{(1-\alpha)+3 / 4} \approx V_{I+} \cdot \frac{4}{7} \sin \left(\alpha \cdot 90^{\circ}\right)
\end{gathered}
$$

which are the sine and cosine weighted versions of the baseband voltage $V_{I+}$. Note that the $3 / 4$ capacitors also have to be emptied of charge before the next cycle begins, in order to preserve input matching.

For a vector modulator the sine and cosine weighted in-phase and quadrature vectors can be used to produce phase shifted signals, as was outlined in section 4.1.1. This principle is reproduced in Fig. 5.9a. The phase shifted vector $V_{0^{\circ}}$ is composed out of the cosine-weighted $V_{I+}$ and sine-weighted $V_{Q+}$. This can be achieved by using the sine/cosine approximation circuit from Fig. 5.8 for both the In-phase and Quadrature signal.

As in the design from the previous chapter, time interleaving can be used to produce the sum of the two weighted vector, as is shown in Fig. 5.9b. Note that for $V_{I+}$ the additional path with $\alpha$ capacitor is not shown for clearity, as well as the $1-\alpha$ capacitor path for $V_{Q+}$. The $25 \%$ duty cycle clock however forces the use of four paths to the output vector instead of the two in the previous design. This is achieved by duplicating each path and clocking by phases advanced by half of the clock period. As a result, the output is presented with a voltage from a 3/4 capacitor during each clock phase, effectively reconstructing a continuous time output waveform.

It is required in a zero-IF architecture to have differential In-phase and Quadrature 


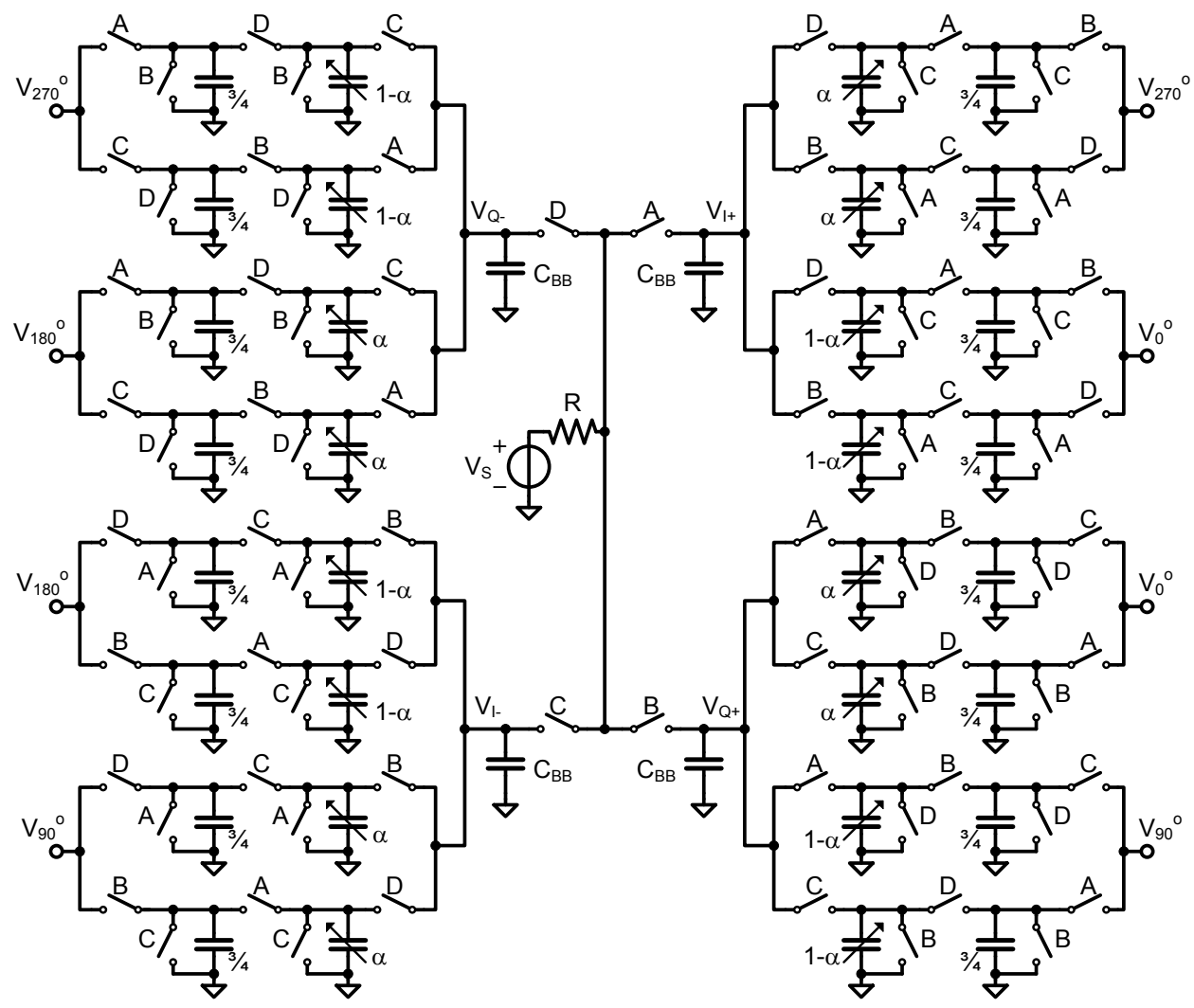

Figure 5.10: Input-matched passive downconverter with phase shifting.

outputs in order to perform image rejection. In a phased array, these I/Q outputs are phase shifted with respect to the mixer I/Q signals. This is achieved by duplicating the phase shifting circuit four times with proper clocking and wiring, with the circuit in Fig. 5.10 as a result. Each mixer output is now equally loaded with the same load capacitor regardless of $\alpha$, and a phase-shifted four-phase output is formed.

With this structure a phase shift between $0^{\circ}$ and $90^{\circ}$, corresponding to a single quadrant in the phasor diagram, can be achieved by changing $\alpha$ between zero and one. This effectively moves capacitance between two parallel branches (Fig. 5.8) with a fixed total capacitance at all times, resulting in input matching for all phase shifter settings. The three other quadrants can be reached by swapping $V_{0^{\circ}}, V_{90^{\circ}}, V_{180^{\circ}}$ and $V_{270^{\circ}}$, enabling the full $0^{\circ}$ to $360^{\circ}$ phase shift range.

Note that compared to the phase shifter in chapter 4 , the $3 / 4$ capacitor is also emptied each cycle. The purpose of this is to eliminate coupling between phase shifters, which is necessary as there is no active intermediate stage to isolate one phase shifter from the other. 


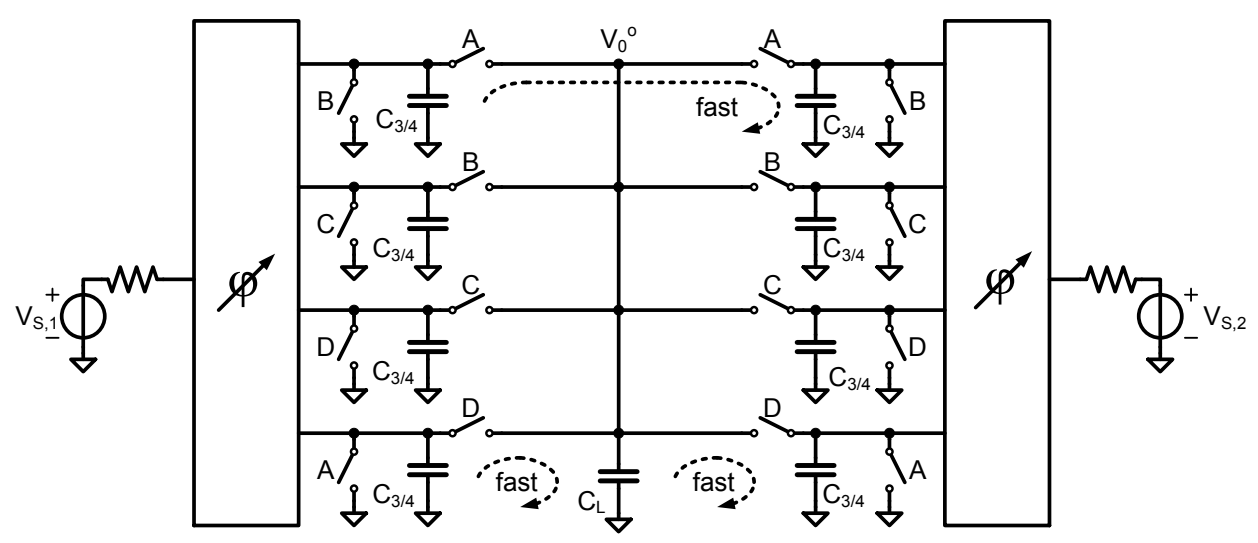

Figure 5.11: Output node summing for 2 elements.

\subsubsection{Element Summing}

In the previous chapter element summing was achieved in the current domain by attaching the output nodes of transconductors together. The passive architecture works with packets of charge, instead of a continuous stream of charge, but the same principle can be applied. Fig. 5.11 illustrates the mechanism that takes place if the element outputs are simply connected together, including the effects of a load capacitor.

First of all, for each clock phase there is a capacitor connected to the output for each element with the charge corresponding to its output voltage. At the moment the switch closes, these capacitors see each other and charge redistribution takes place to arrive at a common voltage. When the associated loop is fast, this voltage settles and charge conservation dictates that the resulting voltage is equal to the average of the respective element voltages.

Moreover, when a load capacitor $C_{L}$ much larger than $C_{3 / 4}$ is present at the output node, there are additional fast loops which deposit charge from $C_{3 / 4}$ to $C_{L}$. In a manner similar to $(5.17)$, each $C_{3 / 4}$ has charge $Q=C_{3 / 4} \cdot V_{O U T}$ available per clock cycle, resulting in an average current $I_{O U T}$. Therefore, from the perspective of $C_{L}$ the summing node present an output resistance $R_{O U T}$ equivalent to:

$$
R_{O U T}=\frac{1}{4 N \cdot C_{3 / 4} \cdot f_{s}}
$$

where $N$ is the number of elements connected to the output node. Together with $C_{L}$ this forms a first-order pole at the output, which can be used as a coarse reconstruction filter for the sample-and-held output waveform. 


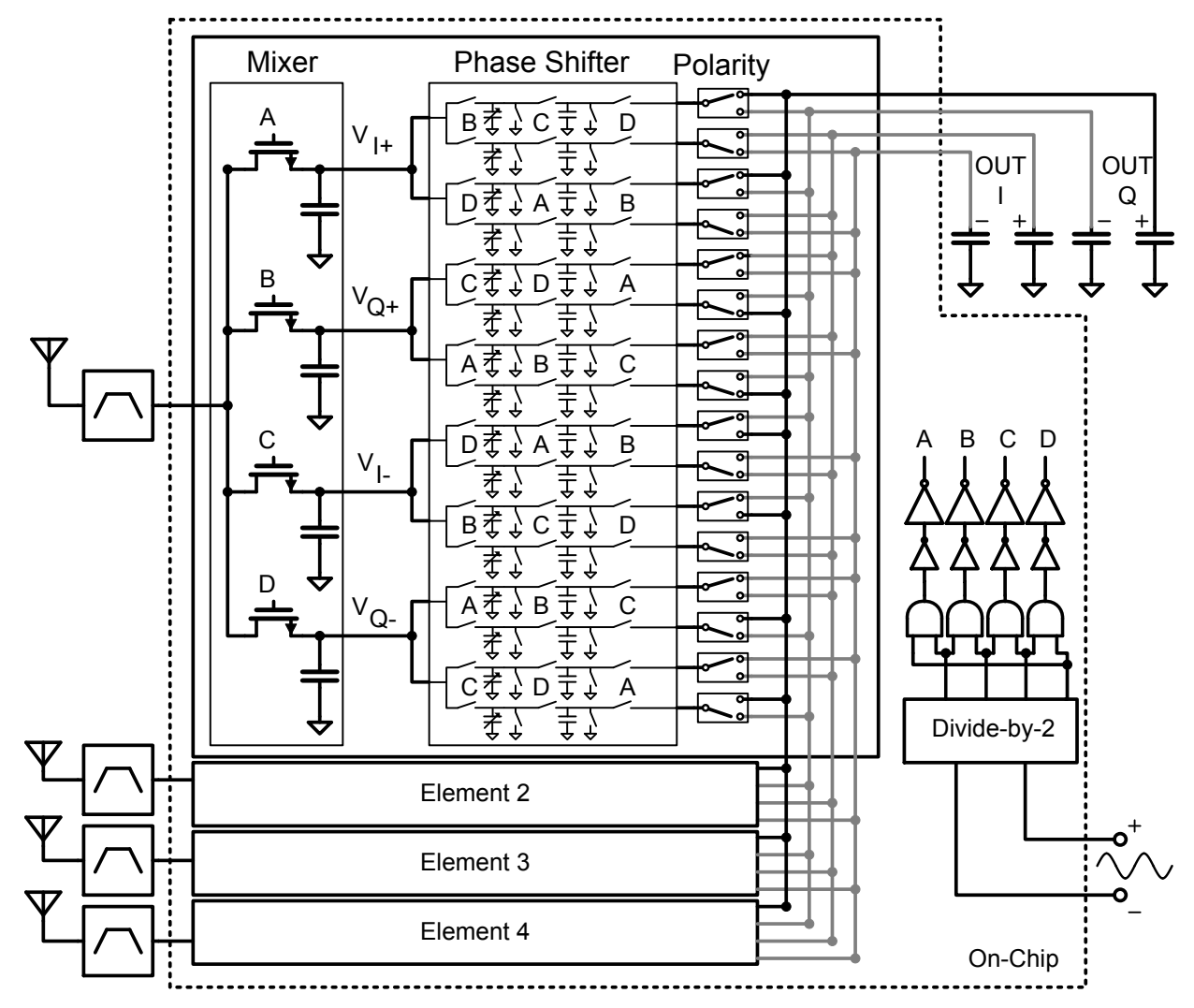

Figure 5.12: All-passive phased-array receiver architecture.

\subsection{Implementation in 65-nm CMOS}

The proposed four-element passive beamforming receiver front-end is shown in Fig. 5.12. This mixer-first architecture loads the passive input mixer with the switchedcapacitor phase shifter to provide input matching at an RF frequency of $3 \mathrm{GHz}$. An $\mathrm{RF}$ pre-filter is necessary to filter out signals at the harmonics of the clock, as is usual for a switching mixer receiver.

Polarity switches at the output of the phase shifters are used to switch between vector modulator quadrants, while the load capacitor on the output node consists of the capacitive input impedance of the active voltage probe used for measurements. An external differential clock is divided by 2 to generate 4 clock phases and passed through AND gates to convert the $50 \%$ duty cyle into a $25 \%$ duty cycle.

The sources and drains of all switches in the chip are biased at ground potential, as there are no active circuit blocks (requiring bias voltage) present in the receiver path. This enables the full swing clock to be driven onto the switch gates directly 


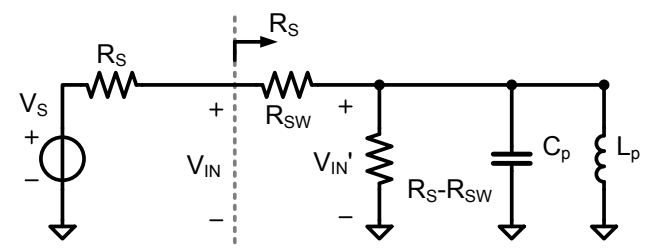

Figure 5.13: Mixer input equivalent model with finite switch resistance.

with inverters, for low switch-on resistance and high linearity.

\subsubsection{Mixer}

Since it is expected that the sample-and-hold noise of the phase shifter is dominant for the system noise figure, the switch-on resistance of the mixer does not have to be dimensioned for noise. However, unlike the implementation in previous chapters, the impedance matching requirements on the mixer result in the conversion loss depending on the switch-on resistance.

In section 5.1.2, the switches were assumed ideal with zero on-resistance. Conceptually, a finite switch on-resistance $R_{S W}$ can be moved in front of the mixer, which again has ideal switches. The input equivalent model of Fig. 5.6 under matched conditions is now modified to the one in Fig. 5.13.

The input voltage $V_{I N}$ (which is half the source voltage $V_{S}$ ) is divided by the resistor ladder before being presented to the idealized input of the mixer:

$$
V_{I N}^{\prime}=V_{I N} \cdot\left(1-\frac{R_{S W}}{R_{S}}\right)
$$

Moreover, the transfer function from the idealized input of the mixer to the down converted baseband signal is sinc $(1 / 4)$, so that the conversion gain inside the mixer pass band is equal to ${ }^{2}$ :

$$
C G_{\text {mixer }}=\frac{1-\frac{R_{S W}}{R_{S}}}{\operatorname{sinc}(1 / 4)}
$$

A choice is made for $10 \Omega$ switch-on resistance, balancing input capacitance (due to switch parasitics) and a $1 \mathrm{~dB}$ conversion loss.

The baseband capacitor $C_{B B}$ determines the dominant pole of the receiver. A $-3 \mathrm{~dB}$ bandwidth of $300 \mathrm{MHz}$ is accomplished by picking $2.5 \mathrm{pF}$ for these capacitors (with a respective $\Gamma$ of 0.1 for $3 \mathrm{GHz} \mathrm{RF}$ ), in order to show of the inherently wideband operation. Of course, $C_{B B}$ can be increased to provide more baseband filtering.

\footnotetext{
${ }^{2}$ It is custom in RF circuits to relate the gain of system to the input-matched voltage $V_{I N}$, which is half of the source voltage $V_{S}$.
} 

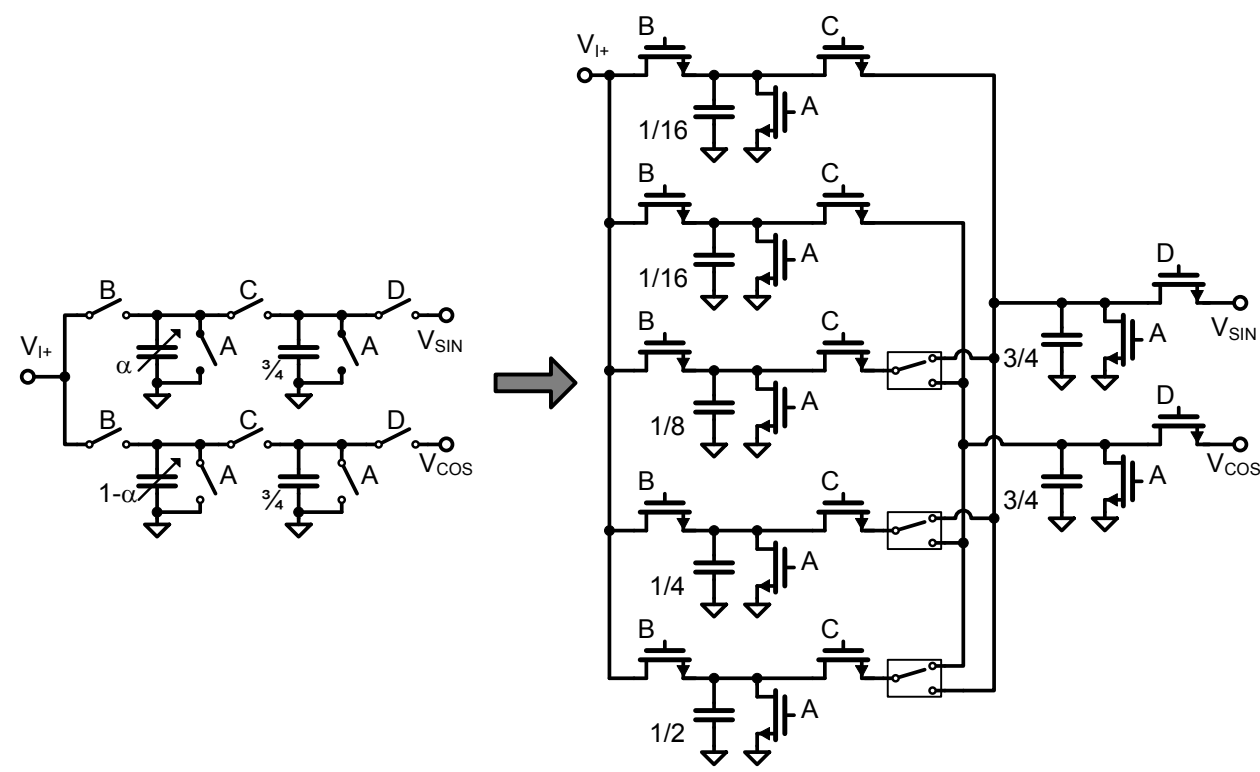

Figure 5.14: Phase shifter slice.

\subsubsection{Phase Shifter Slice}

As was shown in section 5.1.4, the phase shifter charge redistribution slices with $\alpha$ and $1-\alpha$ capacitors can be paired and implemented with a single capacitor bank. The circuit implementation of one such pair is presented in Fig. 5.14, where all switches are implemented with NMOS transistors and all capacitors with metal fringe capacitors. Note the resemblance to the binary weighted capacitor bank in Fig. 4.10, with the difference that capacitance is not connected/disconnected but switched between the two outputs. This ensures that each capacitor carries useful signals and no clock power is wasted to switch capacitors which are not used. As a down-side, there is now no freedom to choose the ratios independently, so no amplitude control is possible without breaking the impedance matching.

From equation (5.17), the value of the 1/16 capacitor for impedance matching at $3 \mathrm{GHz}$ is $32 \mathrm{fF}^{3}$. The switches are dimensioned such that the slowest (fast) loop has a $\Gamma$ slightly over 2 , just inside the sampling region. Circuit simulations indicate that for this case, the performance is hardly degraded compared to higher $\Gamma$ values, while offering a significant dynamic power reduction. It is worth noting that the charge redistribution loop that performs the actual sine and cosine weighting has an intrinsically higher $\Gamma$, because this loop has two roughly equal capacitors in series, resulting in a much lower effective capacitance. Of course, the dominant switch para-

\footnotetext{
${ }^{3} 25 \mathrm{fF}$ was actually implemented due to the $10 \Omega$ mixer switch resistance.
} 


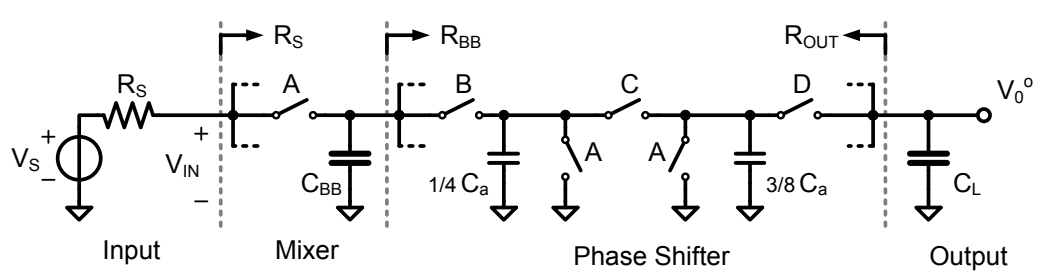

Figure 5.15: Single path from input to output for $\alpha=0.5$.

sitic capacitances are to low impedance nodes and can be absorbed into the functional capacitances, constituting about $20 \%$ of each node's capacitance.

\subsubsection{Conversion Loss and Noise Figure}

For a calculation of the conversion loss, it is easiest to consider the situation with $\alpha=0.5$, as this results in equal signal amplitudes in all parallel branches of the phase shifter. In this case, a single path from input to output can be depicted schematically as in Fig. 5.15. Note that there are three points where multiple polyphase paths branch off or combine together. These are also the points where impedances are defined to determine the coupling between stages.

With the input matched to the source, the input voltage is half the source voltage. To keep to the convention in RF systems, the gain of the total system is referenced to this input voltage. From (5.22) the decision was made for a $1 \mathrm{~dB}$ loss from the input voltage to the mixer capacitor. Then, evaluating (5.19) for $\alpha=0.5$ gives the loss in the phase shifter to be $8 \mathrm{~dB}$. At the output of the phase shifter, half of the time the In-phase signal is present and half of the time the Quadrature signal is present. This interleaving introduces an additional $3 \mathrm{~dB}$ of loss, bringing the total voltage loss from the input to the $0^{\circ}$ output on $12 \mathrm{~dB}$.

From Fig.3.15b, it was found that fast loops have a considerably larger noise figure than slow loops. This is due to the inherently larger noise folding, caused by the substantially larger loop bandwidth. Therefore, the fast loops in the phase shifter can be assumed to be the dominant noise source. Each switch in the phase shifter is associated with a fast loop and will induce a sampled noise voltage on the respective loop capacitor at the moment when the switch opens. This noise voltage is held on the capacitor during the hold interval with length $T_{S H}$, shown schematically in Fig. 5.16 for a single SE kernel. The double-sided PSD of this noise is given by (3.29) where the noise part due to the loop resistance $R$ is only marginal and therefore omitted:

$$
N_{S H}=\frac{k T}{C \cdot f_{s}} \cdot\left(\frac{T_{S H}}{T_{S}}\right)^{2}
$$

The duty cycle is expressed as $T_{S H} / T_{S}$, the length of the hold interval with respect 

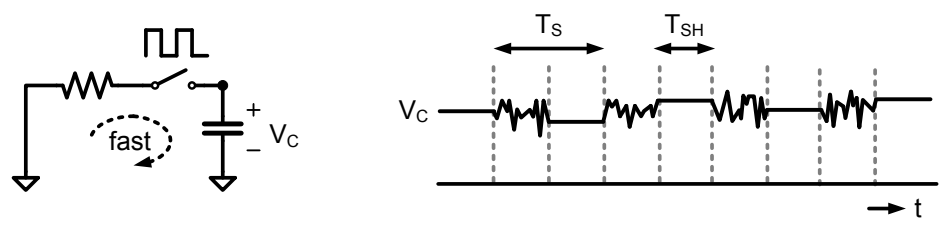

Figure 5.16: Time domain noise in a fast loop.

to the clock period, which is equal to $1 / 4$.

In order to calculate the total output noise, the sample-and-hold noise contribution of each switch has to be calculated and referred to the output. The summed output noise due to internal noise sources can then be expressed as:

$$
N_{o, \text { int }}=1.5 \frac{k T}{C_{a} \cdot f_{s}}
$$

But, $C_{a}$ is fixed to produce impedance matching with the source impedance in expression (5.17), such that a substitution gives:

$$
N_{o, \text { int }}=6 k T R_{s}
$$

Applying to 3.34 (and omitting the output noise due to the source resistance, which is not dominant) gives:

$$
N F \approx 10 \cdot \log \left(4^{2} \cdot \frac{6 k T R_{s}}{\frac{1}{2} k T R_{s}}\right)=23 \mathrm{~dB}
$$

as sensed at a single output phase.

However, these results are for a single output phase. Four output phases are present in the complete system, which are bundled into a differential In-phase and Quadrature output. The sensed outputs are differential and therefore, the conversion loss is decreased to $6 \mathrm{~dB}$. Furthermore, performing image rejection with the fourphase output will increase the output signal-to-noise ratio by $6 \mathrm{~dB}$, as derived by the polyphase multipath noise analysis in section 3.1.5. This improves the system noise figure to $17 \mathrm{~dB}$.

\subsubsection{Clock Generation}

In previous chapters, with clock dividers based on full-swing transmission-gated inverter logic, there is a practical limit for the maximum reference clock frequency that can be handled. Moving from a divide-by-4 to a divide-by-2 architecture maximizes the resulting switching frequency, but in 65-nm CMOS technology this maximum still lies around a $4 \mathrm{GHz}$ divided clock. 


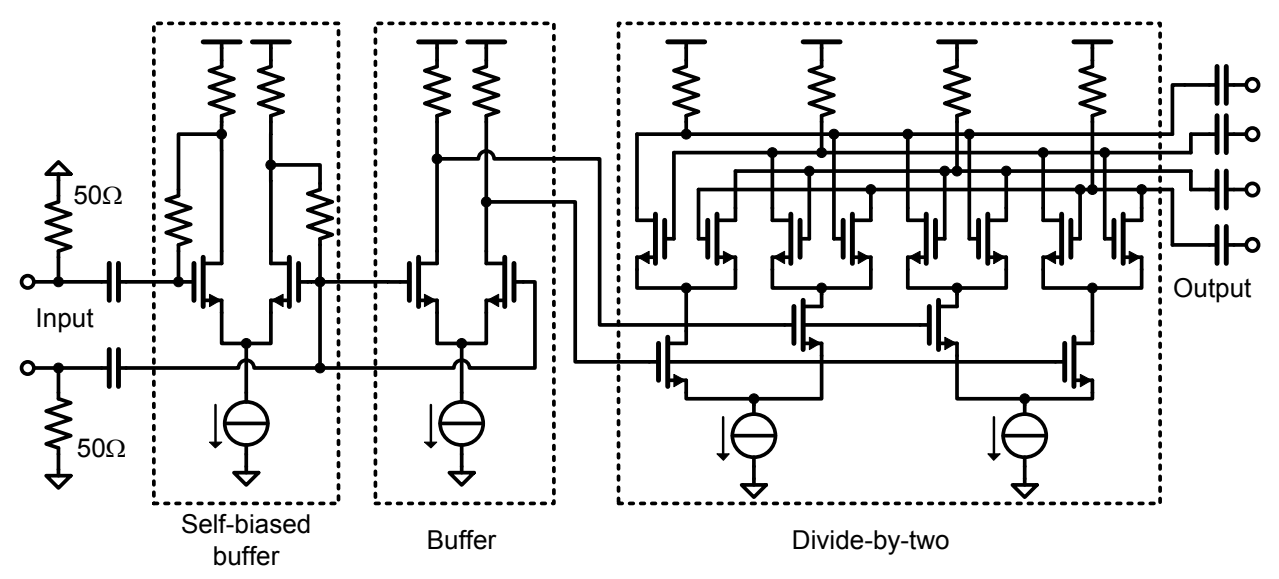

Figure 5.17: Current-Mode-Logic clock divider.

In comparison, a half-swing current mode logic (CML) based divider can handle much higher reference frequencies, mainly because it only uses NMOS devices instead of a NMOS and PMOS mixture. Therefore, the divider in Fig. 5.17 is used for this design. The first stage is a self-biased buffer with AC coupling. It is followed by an intermediate buffer which drives a conventional divide-by-two. The output at half the reference frequency is AC coupled, for coupling into inverters (not shown) which boost the signal from half swing to full swing.

A distinct advantage of this CML topology is the benefit of common-mode rejection in each stage. As a result, the differential reference clock can have a very large phase and gain error, which show up as common-mode components and are suppressed. In measurements, the disconnection of one reference clock input resulted in an only marginal decrease in performance, showing the robustness of this approach.

The stages were dimensioned for a total simulated RMS phase mismatch of $0.7^{\circ}$, resulting in a static power consumption of $15 \mathrm{~mW}$. A mismatch in the clock phases will result in an elliptical distortion of the phase shifter constellation. This distortion introduces both an amplitude and a phase error, effectively spreading the error energy between the two. With the help from equation (4.6) to express the distance in the vector diagram, the expected RMS gain and phase error due to mismatch are:

$$
\begin{aligned}
\sigma_{\varphi} & =0.5^{\circ} \\
\sigma_{A} & =0.08 \mathrm{~dB}
\end{aligned}
$$

\subsubsection{Measurements}

The chip is implemented in 65-nm CMOS and its die photo is shown in Fig. 5.18, active area is $0.18 \mathrm{~mm}^{2}$. Nominal supply voltage of $1.2 \mathrm{~V}$. 


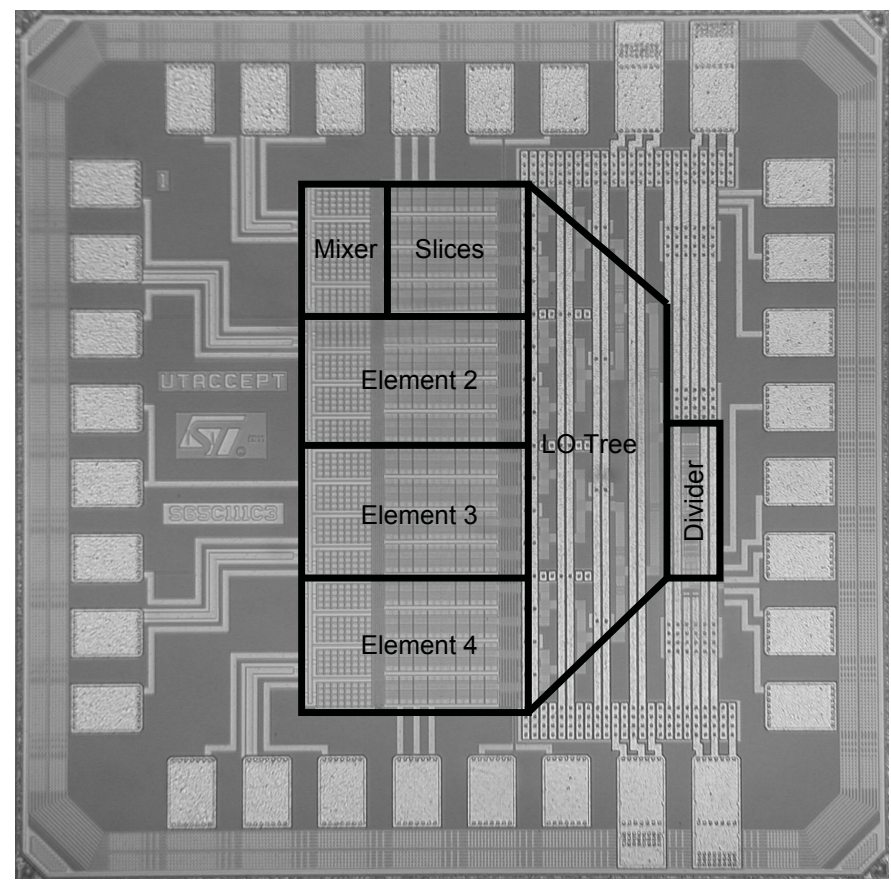

Figure 5.18: Photograph of the $0.9 \mathrm{~mm}$ by $0.9 \mathrm{~mm}$ die.

The measured input matching for a single element is shown in Fig. 5.19, for several LO frequencies. As was expressed in section5.1.2, the input impedance looks like a parallel LC resonant circuits that is centered on the LO frequency. The best matching is achieved for the $3.0 \mathrm{GHz}$ design frequency, where the real part of the input impedance equals $50 \Omega$ as expressed in (5.17). But $\mathrm{S} 11$ better than $-10 \mathrm{~dB}$ is still achieved for a 1.5 to $5.0 \mathrm{GHz}$ band.

A plot of the noise figure, compression point and gain, as measured on a single element input are shown in Fig. 5.20a. The NF on the design frequency equals 18 $\mathrm{dB}$, only $1 \mathrm{~dB}$ off from the theoretical result in section 5.2.3.

Moreover, the input-referred in-band compression point is $2 \mathrm{dBm}$ over the entire frequency band. Simulation indicate that the compression mainly occurs in the mixer. When plotted as a function of frequency offset from the LO in Fig. 5.20b, the compression is seen to increase up to an out-of-band compression point of $12 \mathrm{dBm}$. This power in a $50 \Omega$ impedance would result in $2 \mathrm{~V}$ amplitude swing at the output of the IC, far outside the supply voltage, but the input resistance rolls of to a low value outof-band, thereby reducing the voltage swing at the IC input and therefore increasing the compression point. The plot includes in a dashed line the baseband first-order low pass filtering with $300 \mathrm{MHz}$ bandwidth, illustrating that the compression point follows the filter curve. 


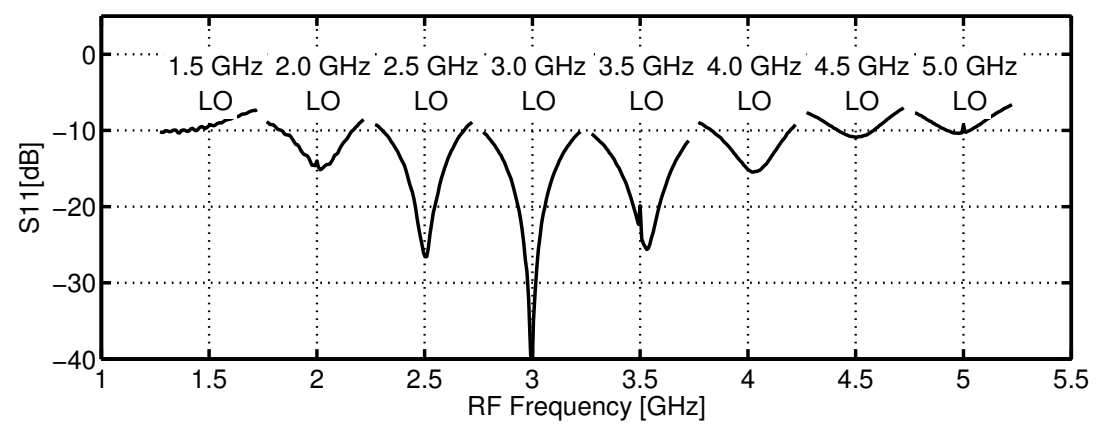

Figure 5.19: Measured S11 vs. clock frequency.

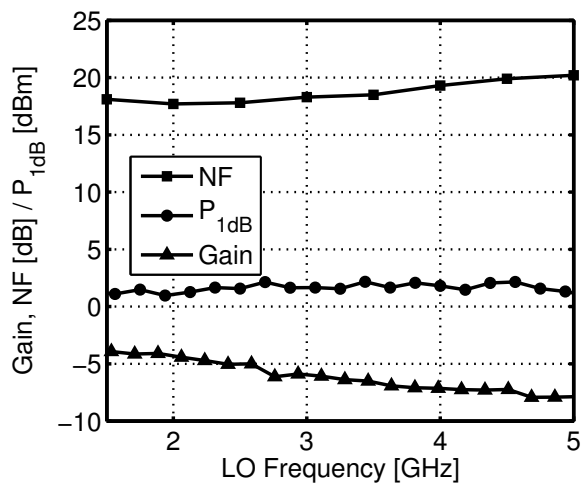

(a)

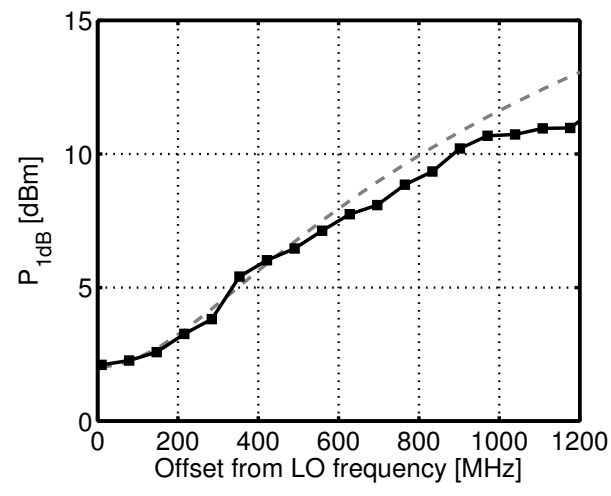

(b)

Figure 5.20: Measured element (a) NF, compression point, gain and (b) compression point vs. offset from LO.

The phase steps and gain errors of the phase shifter are shown in Fig. 5.21, plotted as a function of LO frequency with fixed baseband frequency. Very stable performance over frequency is demonstrated, due to the fact that capacitor ratios determine the phase shifter's transfer function. The RMS phase error and gain error are $2.0^{\circ}$ and 0.2 $\mathrm{dB}$ respectively. A 10-die mismatch measurement reveals that the mismatch between dies and elements is $0.4^{\circ}$ and $0.06 \mathrm{~dB}$ RMS, close to the expected performance from the clock generator.

In order to test the summing node of the phased array, a test setup is made where the signal from an RF generator is split four-ways with power splitters and fed with equal phase into the four RF inputs of the IC. Then, the phased array is programmed to produce beam patterns with a main beam sweeping over angle. This corresponds to increasing the phase shift between elements more and more. As there is a 5-bit 

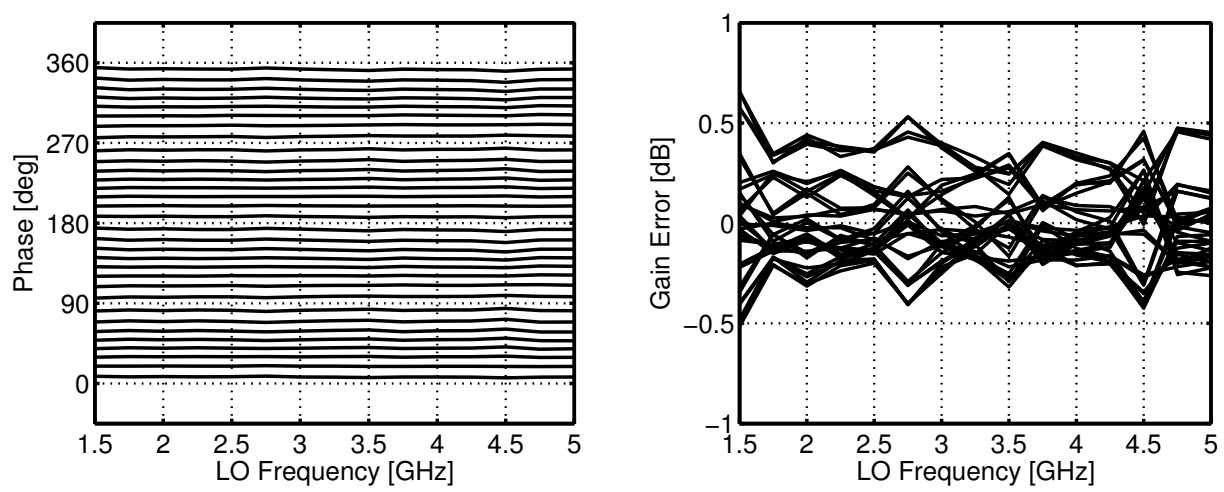

Figure 5.21: Measured element phase shift and gain variations.

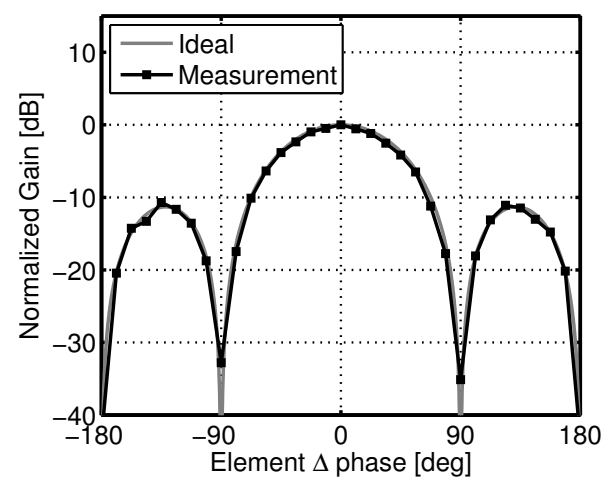

(a)

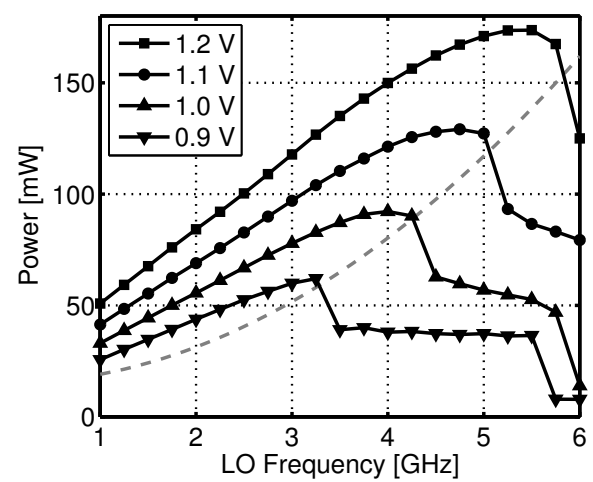

(b)

Figure 5.22: Meaured receiver (a) summation pattern and (b) power consumption vs. supply voltage.

phase shift control, this means that there are 32 main beam positions. When the power is measured at the output, the setup measures one point of each beam pattern, at the broadside direction $(\theta=0)$. Because in sine-space, beam steering results in just a shift of the beam pattern (section 2.1.1), this relatively simple setup measured a quantized version of the actual beam pattern. The resulting measurement is shown in Fig. 5.22a, together with in grey the ideal calculated line. This measurement not only shows the summing capabilities of the receiver, but also confirms the uniformity of the phase steps in the phase shifter.

With the eliminating of active stages in the receiver path, the possibility of supply voltage adaptation opens up. There are no active stages to be biased, and the inverters in the clock path can handle many supply voltages. The biasing for the CML stages 
Table 5.1: Performance Summary

\begin{tabular}{|l|c||l|c|}
\hline Phased Array: & & Single Element: & @ 3.0 GHz RF \\
\hline Technology & $65-\mathrm{nm}$ CMOS & Gain & $-6 \mathrm{~dB}$ \\
\hline Active Area & $0.18 \mathrm{~mm}^{2}$ & Noise Figure & $18 \mathrm{~dB} \mathrm{DSB}$ \\
\hline $\begin{array}{l}\text { Input matched } \\
\text { frequency band }\end{array}$ & $1.5-5.0 \mathrm{GHz}$ & $\begin{array}{l}\text { In-band 1 dB } \\
\text { compression point }\end{array}$ & $2 \mathrm{dBm}$ \\
\hline $\begin{array}{l}\text { Power } \\
\text { consumption @ } 1.2 \mathrm{~V}\end{array}$ & $65-168 \mathrm{~mW}$ & In-band IIP3 & $13 \mathrm{dBm}$ \\
\hline Supply Voltage & $0.9-1.2 \mathrm{~V}$ & In-band IIP2 & $69 \mathrm{dBm}$ \\
\hline Elements & 4 & Phase control & $5 \mathrm{bit}$ \\
\hline Die Size & $0.87 \times 0.87 \mathrm{~mm}$ & Phase error & $2.0^{\circ} \mathrm{RMS}$ \\
\hline $\begin{array}{l}\text { Maximum RF } \\
\text { frequency }\end{array}$ & $5.8 \mathrm{GHz}$ & Amplitude error & $0.2 \mathrm{~dB}$ RMS \\
\hline
\end{tabular}

was designed to follow voltage changes. By bringing down the supply voltage, the power consumption is reduces with the square of the reduction, as is well known in digital design. This is illustrated by the measurement of the chip power consumption in Fig. 5.22b. Lowering the voltage from $1.2 \mathrm{~V}$ to $0.9 \mathrm{~V}$ decreases the power to nearly half, while there is no significant decrease in the phased-array performance.

The consequences of the supply voltage reduction are three-fold. First of all, the maximum frequency of operation is reduced, as the inverters become slower for lower voltages. This is illustrated by the grey line in the figure, which marks the point where the rise- and fall-times are too large to fit within the pulse time of the clock. Second, the reduces overdrive voltage on the mixer switches reduces the compression point, down to $-2 \mathrm{dBm}$ for $1.0 \mathrm{~V}$. Thirdly, the noise figure is hardly affected and increasing by only $0.3 \mathrm{~dB}$ for $1.0 \mathrm{~V}$. This confirms that main source of noise is from the sampling region switched-RC loops, which produce the same noise regardless of switch-on resistance.

The measured performance is summarized in Table 5.1. Noteworthy is the good second-order linearity performance, an in-referred in-band IP2 of $69 \mathrm{dBm}$, due to the absence of active stages in the signal path.

\subsection{Conclusions}

In this chapter a design framework for using coupled sampling and mixing region switched-RC loops has been presented. It was concluded that for coupled sampling loops, the time domain is more useful, while for coupled mixing loops, an analysis in the frequency domain can be used. Moreover, by defining an interface resistance the two types can be coupled together, with the coupling effects calculated for both sides.

With these techniques, a passive phased-array architecture without active stages in 
the signal path has been proposed, which merges the passive mixer and approximatesine phase shifter work together in one design. The coupling between mixer and phase shifter works two-fold, performing impedance matching at the mixer side and providing charge for the phase shifter's charge redistribution process.

The 65-nm CMOS 4-element receiver implementation demonstrates the phasedarray capabilities and the high linearity inherent to this passive design. It was found that the filtering at the input node of the IC results in a very high $12 \mathrm{dBm}$ out-of-band compression point, fitting well to the requirements for interferer rejection. 


\section{Chapter 6}

\section{Conclusions}

\subsection{Summary and Conclusions}

In chapter 1, an introduction to single-antenna and multi-antenna wireless systems was given. The beamforming capabilities of a phased array system and the capacityincreasing capabilities of spatial multiplexing systems were highlighted, along with the intrinsic differences between them. Furthermore, the trends in advanced CMOS have been sketched, along with the difference in device compatibility compared to compound technologies.

In chapter 2, the fundamental properties of linear phased arrays were summarized first. The basic beam pattern was introduced and the effects on it by beamsteering, amplitude tapering and the phase shifter approximation was summarized, along with the degradation due to errors in the array. Next, a framework for including downconversion in the beamforming architecture has been presented, along with transformation methods to move time delay and/or phase shift between the RF, LO and IF domain. It was found that the reallocation of the beamforming to the LO or RF domain can give rise to beam squinting, as is the case with the phase shifter approximation in general array theory. Furthermore, the linearity and sensitivity properties of beamforming circuit implementations were covered, concluding that beamforming can be viewed as a form of impedance scaling.

In chapter 3, the use of switched-RC donwconverters in a mixer-first receiver architecture was analyzed. It was shown that a broad range of switched-RC samplers and mixers can be understood to be an assembly of simple kernels with polyphase clock timing. A unified frequency domain description was derived, highlighting the similar- ity in operation of these circuits. Moreover, it was shown that there are two distinct operating regions of the kernels, depending on the loop bandwidth with respect to the clock frequency and duty cycle. Analysis shows that using a $25 \%$ 
duty cycle switched-RC kernel with low loop bandwidth and a 4-phase clock provides a low fundamental conversion loss and noise figure, while being compatible with a I/Q image-reject architecture. This configura- tion, together with baseband feedback amplifiers, is implemented in a 65nm CMOS implementation and achieves $79 \mathrm{~dB}$ of $\mathrm{SFDR}$ in $1 \mathrm{MHz}$ bandwidth, which is in the range of commercial spectrum analyzers.

In chapter 4, a switched-capacitor vector modulator was presented and used in a single-chip 4-element phased-array receiver. A rational approximation of the sine and cosine was proposed in order to generate the non-uniformly quantized weights needed for uniform phase steps. This approximate-sine weighting is mapped on a charge-redistribution circuit and results in all vector modulator settings being used for effective phase shifts. As the phase shift depends on the ratio between capacitors, it is very well controlled. The 65 -nm CMOS implementation with 5 bit phase control and 3 bit amplitude control in the vector modulator achieves an RMS systematic phase and gain error of $1.4^{\circ}$ and $0.4 \mathrm{~dB}$ respectively. Applications in crowded frequency bands, like the $2.4 \mathrm{GHz}$ ISM band, are enabled by the high $-1 \mathrm{dBm}$ IIP3. Moreover, a spatial interference rejection $>20 \mathrm{~dB}$ through nulling was demonstrated, to lower the linearity requirements on the baseband receiver part.

In chapter 5, a design framework for using coupled sampling and mixing region switched-RC loops has been presented. It was concluded that for coupled sampling loops, the time domain is more useful, while for coupled mixing loops, an analysis in the frequency domain can be used. Moreover, by defining an interface resistance the two types can be coupled together, with the coupling effects calculated for both sides. With these techniques, a passive phased-array architecture without active stages in the signal path has been proposed, which merges the passive mixer and approximatesine phase shifter work together in one design. The coupling between mixer and phase shifter works two-fold, performing impedance matching at the mixer side and providing charge for the phase shifter's charge redistribution process. The $65-\mathrm{nm}$ CMOS 4-element receiver implementation demonstrates the phased-array capabilities and the high linearity inherent to this passive design. It was found that the filtering at the input node of the IC results in a very high $12 \mathrm{dBm}$ out-of-band compression point, fitting well to the requirements for interferer rejection.

\subsection{Original Contributions}

- The rigorous mathematical derivation of the transfer function for a switched-RC kernel, unifying the description of mixer and sampler circuits. (chapter 3)

- The concept and design of passive mixer-first receiver architectures. (chapters 3 and 5 ) 
- A rational approximation for the sine and cosine needed in a vector modulator phase shifter, which is equal to the transfer function of a simple charge redistribution switched capacitor circuit. (chapter 4)

- The design of a switched-RC vector modulator, operating at the same frequency as the passive mixer, thereby avoiding extra folding of the frequency spectrum. (chapter 4)

- A design method for coupling mixing and sampling region switched-RC kernels through the definition of the interface resistance. (chapter 5)

- The design of a fully passive beamforming receiver, achieving simultaneous input matching, downconversion, phase shifting and element summing. (chapter 5)

\subsection{Future Work}

The increasing integration level in CMOS, so essential for its economic success, has a profound effect on the development of circuits for wireless communications. Not only does the smaller feature size enable faster switching speeds, but also the partitioning of a receiver in lots of multiple slices, for example in the multi-path polyphase technique (chapter 3). As a result, looking at a receiver frontend as a whole is preferred over the classical partitioning in separate LNA, mixer, baseband filter, etc.

This is illustrated by the significant research attention for passive mixer-first receiver architectures (chapter 3) in the past few years, for applications in low-power wireless sensor nodes [47] and software defined radio [58] [64]. The inherently high linearity and flexibility in RF frequency of these architectures lends itself well for these applications, albeit at the cost of a somewhat higher noise figure than tuned receivers. Recently, a passive mixer-first receiver with noise canceling has been presented [65], bringing down the noise figure to $2 \mathrm{~dB}$.

In the design of the fully passive beamforming receiver (chapter 5) it was derived that the passive mixer transforms the baseband lowpass filter to an RF bandpass filter. This principle was already known in the 1960s [59], but its switched-RC implementation shows great promise for providing high-Q integrated filters in CMOS. Implementations of $2^{\text {nd }}$ order [66] [67] and $4^{\text {th }}$ order [68] bandpass butterworth filters with Q values as high as 57 have been published.

There has been some further research on receiver beamforming in the lower frequency bands. A $4 \mathrm{GHz}$ beamformer in 90-nm CMOS [69] performs the phase shifting in the LO domain (chapter 2) by interpolating between the phases of a multi-phase clock, exploiting the good timing accuracy of clocks in CMOS. In 0.13- $\mu \mathrm{m}$ CMOS, a 5-6 GHz 8-element Butler matrix has been presented [70], which is able to form 8 beams simultaneously. 
Looking at the work presented in this thesis, there are several directions for future innovations. The presented beamforming receivers (chapters 4 and 5) are based on vector modulation with sampling region switched-RC networks. In the mathematical analysis of switched-RC kernels (chapter 3) it was derived that the mixing region has a significantly lower noise contribution compared to the sampling region. Therefore, a design utilizing only mixing region kernels is expected to have a much lower noise figure. One obstacle in the implementation of such a design is the amplitude weighting in the vector modulator. Changing the capacitance in coupled mixing region RC loops only results in changing the bandwidth. For amplitude weighting, it is necessary to change the effective resistance, i.e. duty cycle times resistance, of the loop. One prospect would be to change the duty cycle with accurate timing in the clocks.

Power consumption is always important for mobile devices. The presented designs offer state-of-the-art performance, but at the cost of significantly higher power consumption than classical RF frontends. It is expected that wideband / widely-tunable frontends dissipate more power than tuned frontends, but some power savings are always welcome. A large portion of the power consumption of the switched-RC designs in this thesis is coming from driving the MOSFET switches. By applying resonant clocking, some of the energy can be reused from clock cycle to clock cycle. This limits the clock waveform to a sinusoid, the effect of which on circuit performance has to be evaluated. Of course, power savings can also be reached by switching to circuit topologies which require fewer switches. Also in this respect, an all-mixing-region switched-RC beamforming design looks promising.

Another open end is how amplitude control can be build into the fully passive beamforming receiver (chapter 5). This design depends on the precise dissipation of signal energy in the vector modulator to achieve impedance matching. For lower amplitude settings, the vector modulator does not dissipate enough energy, effectively raising the input impedance. One solution is to enable extra dissipation switches for lower amplitudes settings.

Finally, it must be mentioned that no mathematical analysis of distortion in the switched-RC circuits in this thesis is given. The circuit simulations indicate that with a clock swing of the full supply voltage on the gate of the MOSFET switches, high linearity figures are reliable achieved. In fact, the two mixed active/passive designs (chapters 3 and 4 ) show that the linearity is limited by the active circuits. But still, it would be insightful to analyze what is causing the linearity limitations in the passive circuits and how these limitations can eventually be mitigated. 


\section{Appendix A}

\section{Switched-RC Kernel Analysis}

In this appendix, Linear Periodically Time Variant analysis is performed on the switched-RC kernels to determine their exact harmonic transfer functions. Also, approximate expressions for the mixing and sampling region are derived.

\section{A.1 LPTV Calculation Method}

For the LPTV analysis, the timing definitions from Opal [71] in Fig. A.1a are used, where:

$$
\sigma_{0}=0, \quad \sigma_{k}=\sum_{i=1}^{k} \tau_{i}, \quad k=1 . . K .
$$

The switching pattern is periodic about time $T_{s}$ (having a frequency of $f_{s}=1 / T_{s}$ ) and defines $K$ intervals during which the system has a valid LTI state space description. The $k$-th interval is defined during time $n T_{s}+\sigma_{k}<t<n T_{s}+\sigma_{k+1}$ (where $n$ is an integer).

Ström and Signell made three observations [51]. First, the response in an interval depends only on the input stimulus and initial interval conditions:

$$
\begin{aligned}
& \frac{d}{d t} v_{o}(t)=A_{k} v_{o}(t)+B_{k} v_{i}(t), \\
& n T_{s}+\sigma_{k-1} \leq t<n T_{s}+\sigma_{k},
\end{aligned}
$$

where $v_{i}(t)$ is the input voltage, $v_{o}(t)$ is the output voltage, and $A_{k}$ and $B_{k}$ are the state-space parameters defining this first order system. Secondly, if $v_{o, k}(t)$ is defined as being equal to zero outside and equal to the output voltage inside the $k$-th interval (Fig. A.1b):

$$
v_{o, k}(t)=v_{o}(t) \cdot w_{k}(t)
$$




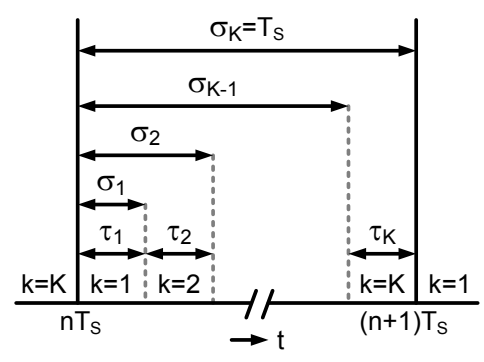

(a)
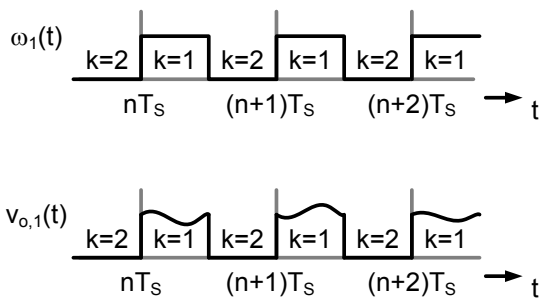

(b)

Figure A.1: (a) timing diagram of the intervals with (b) example of $w_{1}(t)$ and $v_{o, 1}(t)$.

$$
w_{k}(t)= \begin{cases}1 & , n T_{s}+\sigma_{k-1} \leq t<n T_{s}+\sigma_{k} \\ 0 & , \text { elsewhere }\end{cases}
$$

then the output is the sum of all $v_{o, k}(t)$ :

$$
v_{o}(t)=\sum_{k=1}^{K} v_{o, k}(t) .
$$

And finally, the state space response (A.2) can be made zero outside the interval by disconnecting the input source and subtracting final conditions at the interval end ([51] eqn. 7):

$$
\begin{aligned}
& \frac{d}{d t} v_{o, k}(t)=A_{k} v_{o, k}(t)+B_{k} v_{i, k}(t)+ \\
& \sum_{n=-\infty}^{\infty}\left[v_{o}(t) \delta\left(t-n T_{s}-\sigma_{k-1}\right)-v_{o}(t) \delta\left(t-n T_{s}-\sigma_{k}\right)\right], \\
& \quad-\infty<t<\infty,
\end{aligned}
$$

where

$$
v_{i, k}(t)=v_{i}(t) \cdot w_{k}(t)
$$

and $\delta(t)$ is the Dirac delta function.

Because (A.6) is valid for all $t$, the Fourier transform (denoted by $\mathcal{F}()$ ) can be used. The Fourier transforms of (A.6) and (A.5) are:

$$
\begin{array}{r}
\left(j 2 \pi f-A_{k}\right) V_{o, k}(f)=B_{k} \cdot \mathcal{F}\left(v_{i}(t) \cdot w_{k}(t)\right)+ \\
\sum_{n=-\infty}^{\infty}\left[\mathcal{F}\left(v_{o}(t) \cdot \delta\left(t-n T_{s}-\sigma_{k-1}\right)\right)\right. \\
\left.\quad-\mathcal{F}\left(v_{o}(t) \cdot \delta\left(t-n T_{s}-\sigma_{k}\right)\right)\right]
\end{array}
$$


and

$$
V_{o}(f)=\sum_{k=1}^{K} V_{o, k}(f) .
$$

The multiplication of $w_{k}(t)$ and $v_{i}(t)$ becomes a convolution in the frequency domain resulting in ([49] eqn. $6.20+5.13)$ :

$$
\begin{aligned}
& \mathcal{F}\left(v_{i}(t) \cdot w_{k}(t)\right)= \\
& \sum_{n=-\infty}^{\infty} \frac{1-e^{-j 2 \pi n f_{s} \tau_{k}}}{j 2 \pi n} e^{-j 2 \pi n f_{s} \sigma_{k-1}} V_{i}\left(f-n f_{s}\right) .
\end{aligned}
$$

To obtain a closed form expression for the transfer function, we want to express the sampled output voltage terms in (A.8) as a function of the input voltage. Suppose there is a function $G_{k}(f)$ such that the output voltage at switching moment $t=$ $n T_{s}+\sigma_{k}$ can be expressed as:

$$
\begin{aligned}
& \sum_{n=-\infty}^{\infty} \mathcal{F}\left(v_{o}(t) \delta\left(t-n T_{s}-\sigma_{k}\right)\right)= \\
& \sum_{n=-\infty}^{\infty}\left[G_{k}(f) \cdot \mathcal{F}\left(v_{i}(t)\right)\right] * \delta\left(f-n f_{s}\right) \cdot f_{s} e^{-j 2 \pi n f_{s} \sigma_{k}},
\end{aligned}
$$

where the $*$ operator is the convolution integral ([49] eqn. 3.18). Working out the Fourier transform ([49] eqn. $12.7+5.13)$ :

$$
\begin{aligned}
& \sum_{n=-\infty}^{\infty} \mathcal{F}\left(v_{o}(t) \delta\left(t-n T_{s}-\sigma_{k}\right)\right)= \\
& \quad \sum_{n=-\infty}^{\infty} G_{k}\left(f-n f_{s}\right) \cdot f_{s} e^{-j 2 \pi n f_{s} \sigma_{k}} V_{i}\left(f-n f_{s}\right) .
\end{aligned}
$$

We will show that $G_{k}(f)$ does exist for the SE and DI kernels.

After filling in the transformed terms, (A.8) becomes:

$$
\begin{aligned}
& V_{o, k}(f)=\sum_{n=-\infty}^{\infty} H_{n, k}(f) V_{i}\left(f-n f_{s}\right) \\
& H_{n, k}(f)=\frac{1}{j 2 \pi f-A_{k}}[ B_{k} \frac{1-e^{-j 2 \pi n f_{s} \tau_{k}}}{j 2 \pi n} e^{-j 2 \pi n f_{s} \sigma_{k-1}} \\
&+f_{s} G_{k-1}\left(f-n f_{s}\right) e^{-j 2 \pi n f_{s} \sigma_{k-1}} \\
&\left.-f_{s} G_{k}\left(f-n f_{s}\right) e^{-j 2 \pi n f_{s} \sigma_{k}}\right] .
\end{aligned}
$$

According to (A.9) the total HTFs are then:

$$
H_{n}(f)=\sum_{k=1}^{K} H_{n, k}(f) .
$$



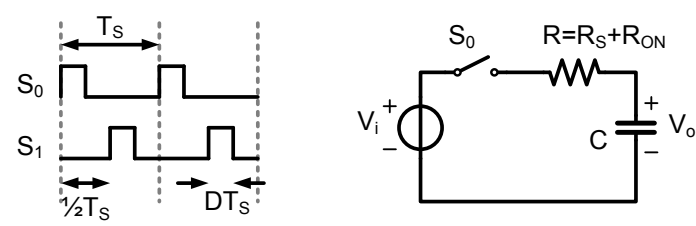

Figure A.2: Single-ended (SE) kernel.

The substitution of (A.13) into (A.14) is in the form of the HTFs definition (3.2) if the following notation is introduced:

$$
f_{o}=f \quad f_{i}=f-n f_{s} .
$$

We see that $G_{k}$ must have the input frequency $f_{i}$ as argument.

\section{A.2 SE and DI Kernel Calculation}

The SE kernel (Fig. A.2) has two intervals. Linear analysis reveals that the switch-on interval $(k=1)$ has the state space description:

$$
\frac{d}{d t} v_{o}(t)=-\frac{1}{R C} v_{o}(t)+\frac{1}{R C} v_{i}(t), \quad n T_{s} \leq t<n T_{s}+\sigma_{1}
$$

so $A_{1}=-\frac{1}{R C} \equiv-2 \pi f_{r c}$ and $B_{1}=\frac{1}{R C} \equiv 2 \pi f_{r c}$. The switch-of interval $(k=2)$ has the state space description:

$$
\frac{d}{d t} v_{o}(t)=0, \quad n T_{s}+\sigma_{1} \leq t<(n+1) T_{s}
$$

so $A_{2}=0$ and $B_{2}=0$.

The switching-moment transfer functions $G_{k}\left(f_{i}\right)$ are determined for the SE kernel in Fig. 3.7b. During an interval the LTI system response includes a zero-input term and a zero-initial-value term ([49] eqn. $8.29+$ eqn. 8.41):

$$
\begin{aligned}
v_{o, k}(t)= & \phi_{k}\left(t-t_{0}\right) v_{o, k}\left(t_{0}\right) \\
& +B_{k} \int_{t_{0}}^{t} \phi_{k}(t-\tau) v_{i}(\tau) d \tau \\
\phi_{k}(t)= & e^{A_{k} t}
\end{aligned}
$$

For sinusoidal input $\left(v_{i}(t)=e^{j 2 \pi f t}\right)$ the solution can be calculated directly. During interval 1:

$$
\begin{aligned}
v_{o, 1}(t)= & e^{-2 \pi f_{r c}\left(t-t_{0}\right)} v_{o, 1}\left(t_{0}\right) \\
& +\frac{1}{1+j \frac{f}{f_{r c}}}\left[e^{j 2 \pi f\left(t-t_{0}\right)}-e^{-2 \pi f_{r c}\left(t-t_{0}\right)}\right] e^{j 2 \pi f t_{0}}
\end{aligned}
$$


and interval 2:

$$
v_{o, 2}(t)=v_{o, 2}\left(t_{0}\right) .
$$

Filling in $t_{0}=n T_{s}$ and $t=n T_{s}+\sigma_{1}$ into (A.19) gives the output voltage at the interval end $v_{o, 1}\left(n T_{s}+\sigma_{1}\right)$ given the input and the initial value $v_{o, 1}\left(n T_{s}\right)$. Since the output voltage is continuous, the initial value of an interval is equal to the final value of the previous interval:

$$
\begin{aligned}
& v_{o, 2}\left(n T_{s}+\sigma_{1}\right)=\lim _{t^{\prime} \uparrow \sigma_{1}} v_{o, 1}\left(n T_{s}+t^{\prime}\right) \\
& v_{o, 1}\left((n+1) T_{s}\right)=\lim _{t^{\prime} \uparrow \sigma_{2}} v_{o, 2}\left(n T_{s}+t^{\prime}\right) .
\end{aligned}
$$

Furthermore, (A.20) gives:

$$
v_{o, 2}\left(n T_{s}+\sigma_{1}\right)=\lim _{t^{\prime} \uparrow \sigma_{2}} v_{o, 2}\left(n T_{s}+t^{\prime}\right) .
$$

By chaining these expressions, the output voltage after a full cycle can be expressed as:

$$
\begin{aligned}
v_{o}\left((n+1) T_{s}\right)= & e^{-2 \pi f_{r c} \tau_{1}} v_{o}\left(n T_{s}\right) \\
& +\frac{1}{1+j \frac{f}{f_{r c}}}\left[e^{j 2 \pi f \tau_{1}}-e^{-2 \pi f_{r c} \tau_{1}}\right] e^{j 2 \pi f n T_{s}} .
\end{aligned}
$$

This result can be viewed as a difference equation with a solution that consists of a steady state and a transient response. For a frequency domain description the transient response can be discarded. With the Z-transform it can be calculated that a difference equation of the form:

$$
v_{o}\left((n+1) T_{s}\right)=\alpha \cdot v_{o}\left(n T_{s}\right)+\beta \cdot e^{j 2 \pi f n T_{s}}
$$

has the steady state solution ([49] eqn. 13.32):

$$
v_{o}\left(n T_{s}\right)=\frac{\beta}{e^{j 2 \pi f T_{s}}-\alpha} \cdot e^{j 2 \pi f n T_{s}}
$$

Applying (A.25) to (A.23) gives:

$$
v_{o}\left(n T_{s}\right)=\left[\frac{e^{j 2 \pi f \tau_{1}}-e^{-2 \pi f_{r c} \tau_{1}}}{e^{j 2 \pi f T_{s}}-e^{-2 \pi f_{r c} \tau_{1}}} \frac{1}{1+j \frac{f}{f_{r c}}}\right] e^{j 2 \pi f n T_{s}},
$$

where the term between brackets is defined as $G_{0}(f)$. We will now show that this is indeed the $G_{0}$ as defined in (A.11).

Equation (A.26) is still in the discrete time domain and gives the output voltage sampled at the switching moments. By inserting delta impulse functions, (A.26) is brought into the continuous time domain:

$$
\sum_{n=-\infty}^{\infty} v_{o}(t) \delta\left(t-n T_{s}\right)=\sum_{n=-\infty}^{\infty} G_{0}(f) \cdot e^{j 2 \pi f t} \delta\left(t-n T_{s}\right)
$$


The above equation gives the output at the switching moments for a single input sinusoid, represented by $e^{j 2 \pi f t}$. After replacing $e^{j 2 \pi f t}$ by the more generic notation $v_{i}(t)$, we can take the Fourier transform of (A.27), resulting in:

$$
\begin{aligned}
\sum_{n=-\infty}^{\infty} \mathcal{F} & \left(v_{o}(t) \delta\left(t-n T_{s}\right)\right)= \\
& \sum_{n=-\infty}^{\infty}\left[G_{0}(f) \cdot \mathcal{F}\left(v_{i}(t)\right)\right] * \delta\left(f-n f_{s}\right) \cdot f_{s} .
\end{aligned}
$$

This was derived for $v_{i}(t)$ being a single sinusoidal input, but because the system is linear, it actually holds for any input signal $v_{i}(t)$.

Equation (A.28) corresponds to the definition of $G_{0}$ in (A.11). The same can be done for $v_{o}\left(n T_{s}+\sigma_{1}\right)$ and $v_{o}\left(n T_{s}+\sigma_{2}\right)$ to find:

$$
\begin{aligned}
& G_{1}(f)=G_{0}(f) e^{j 2 \pi f \tau_{2}} \\
& G_{2}(f)=G_{0}(f) .
\end{aligned}
$$

For the two intervals, (A.13) is evaluated by inserting $A_{k}, B_{k}$ and $G_{k}$ :

$$
\begin{aligned}
H_{n, 1}(f)= & \frac{f_{r c}}{j f+f_{r c}} \frac{1-e^{-j 2 \pi n f_{s} \tau_{1}}}{j 2 \pi n} \\
& -\frac{j f}{j f+f_{r c}} f_{s} \frac{e^{j 2 \pi f \tau_{2}}-1}{j 2 \pi f} G_{0}\left(f-n f_{s}\right) \\
H_{n, 2}(f)= & f_{s} \frac{e^{j 2 \pi f \tau_{2}}-1}{j 2 \pi f} G_{0}\left(f-n f_{s}\right) .
\end{aligned}
$$

Summing according to (A.14) and applying

$$
1-\frac{j f}{j f+f_{r c}}=\frac{f_{r c}}{j f+f_{r c}}
$$

gives the HTFs, which are a function of the output frequency $f_{o}=f$. According to (A.15):

$$
\begin{aligned}
& H_{n}\left(f_{o}\right)= \\
& \frac{f_{r c}}{j f_{o}+f_{r c}}\left[\frac{1-e^{-j 2 \pi n f_{s} \tau_{1}}}{j 2 \pi n}+f_{s} \frac{e^{j 2 \pi f_{o} \tau_{2}}-1}{j 2 \pi f_{o}} G_{0}\left(f_{o}-n f_{s}\right)\right] .
\end{aligned}
$$

This expression can be simplified. The duty cycle $D$ is the duration of the first interval relative to the period time:

$$
D=\frac{\tau_{1}}{T_{s}}=\tau_{1} f_{s}, \quad(1-D)=\frac{\tau_{2}}{T_{s}}=\tau_{2} f_{s} .
$$

So that the SE kernel transfer function becomes:

$$
H_{n}\left(f_{o}\right)=\frac{1}{1+j \frac{f_{o}}{f_{r c}}} \cdot\left[\frac{1-e^{-j 2 \pi D n}}{j 2 \pi n}+\frac{e^{j 2 \pi(1-D) f_{o} / f_{s}}-1}{j 2 \pi f_{o} / f_{s}} G_{0}\left(f_{o}-n f_{s}\right)\right]
$$



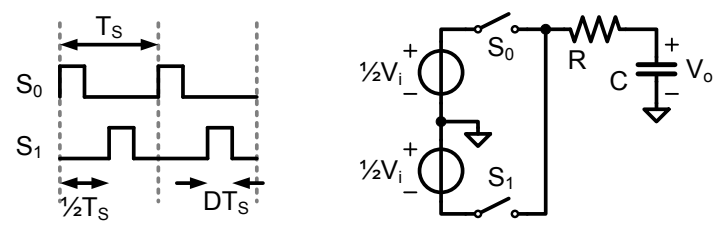

Figure A.3: Differential (DI) kernel.

For the DI kernel in Fig. A.3, the same procedure is used. The DI kernel has four intervals. The first interval $\left(n T_{s} \leq t<(n+D) T_{s}\right)$ has duty cycle $D$, with:

$$
\frac{d}{d t} v_{o}(t)=-\frac{1}{R C} v_{o}(t)+\frac{1}{2 R C} v_{i}(t)
$$

The second interval $\left((n+D) T_{s} \leq t<\left(n+\frac{1}{2}\right) T_{s}\right)$ ends after half the period time :

$$
\frac{d}{d t} v_{o}(t)=0
$$

In the third interval $\left(\left(n+\frac{1}{2}\right) T_{s} \leq t<\left(n+\frac{1}{2}+D\right) T_{s}\right)$ the negative input is connected:

$$
\frac{d}{d t} v_{o}(t)=-\frac{1}{R C} v_{o}(t)-\frac{1}{2 R C} v_{i}(t)
$$

And for the final interval $\left(\left(n+\frac{1}{2}+D\right) T_{s} \leq t<(n+1) T_{s}\right)$ :

$$
\frac{d}{d t} v_{o}(t)=0
$$

The calculation of $G_{k}$ is very similar to the SE kernel case.

$$
G_{0}\left(f_{i}\right)=-\frac{e^{j 2 \pi D f_{i} / f_{s}}-e^{-2 \pi D f_{r c} / f_{s}}}{e^{j 2 \pi \frac{1}{2} f_{i} / f_{s}}+e^{-2 \pi D f_{r c} / f_{s}}} \frac{1}{1+j \frac{f_{i}}{f_{r c}}} .
$$

So that the DI kernel transfer function becomes:

$$
H_{n}\left(f_{o}\right)=\frac{\frac{1}{2}-\frac{1}{2} e^{-j 2 \pi \frac{1}{2} n}}{1+j \frac{f_{o}}{f_{r c}}} \cdot\left[\frac{1-e^{-j 2 \pi D n}}{j 2 \pi n}+\frac{e^{j 2 \pi(1-D) f_{o} / f_{s}}-1}{j 2 \pi f_{o} / f_{s}} G_{0}\left(f_{o}-n f_{s}\right)\right]
$$

\section{A.3 Derivation of Kernel Approximations}

\section{A.3.1 Mixing Region}

For the mixing region, defined as $\Gamma \ll 2$, the limit of $\Gamma \rightarrow 0$ is calculated. In order to get a meaningful limit result, we need to scale $f_{i}$ appropriately with $D f_{r c}$, namely 
as $f_{i}=a D f_{r c}-n f_{s}$ for constant $a$; otherwise, the limit of the HTFs is zero at noninteger $f_{i}$. The scaling reflects the fact that the peaks around integer $f_{i}$ in Fig. $3.8 \mathrm{~b}$ become narrower as $D f_{r c}$ decreases. Rewriting (3.10) in terms of $a$ :

$$
\begin{aligned}
& G_{S E}\left(a D f_{r c}-n f_{s}\right)= \\
& \quad \frac{e^{j 2 \pi D\left(a D f_{r c} / f_{s}-n\right)}-e^{-2 \pi D f_{r c} / f_{s}}}{e^{-j 2 \pi n} e^{j 2 \pi a D f_{r c} / f_{s}}-e^{-2 \pi D f_{r c} / f_{s}}} \cdot \frac{1}{1+j \frac{a D f_{r c} / f_{s}-n}{f_{r c} / f_{s}}} .
\end{aligned}
$$

We first consider the limit of small $f_{r c}$ at constant $D$ and $n \neq 0$ :

$$
\begin{aligned}
& \lim _{f_{r c}^{\prime} \rightarrow 0} G_{S E}\left(a D f_{r c}-n f_{s}\right) \\
& =\lim _{f_{r c}^{\prime} \rightarrow 0} \frac{e^{-j 2 \pi D n}-e^{-2 \pi D f_{r c} / f_{s}}}{1 \cdot e^{j 2 \pi a D f_{r c} / f_{s}}-e^{-2 \pi D f_{r c} / f_{s}}} \cdot \frac{1}{1+j a D-j \frac{n}{f_{r c} / f_{s}}} \\
& =\lim _{f_{r c}^{\prime} \rightarrow 0} \frac{e^{-j 2 \pi D n} \cdot 1-1}{\left(1+j 2 \pi a D f_{r c} / f_{s}\right)-\left(1-2 \pi D f_{r c} / f_{s}\right)+\mathcal{O}\left(f_{r c}^{2} / f_{s}{ }^{2}\right)} \cdot \frac{f_{r c} / f_{s}}{-j n} \\
& =\frac{1}{j a+1} \operatorname{sinc}(D n) e^{-j \pi D n} .
\end{aligned}
$$

Next, a similar calculation gives the limit for small $D$ at constant $f_{r c}$ and $n \neq 0$ :

$$
\lim _{D \rightarrow 0} G_{S E}\left(a D f_{r c}-n f_{s}\right)=\frac{1}{j a+1} .
$$

Calculations for $n=0$ yield the same results. Comparing both limits, we can conclude that for small values of $\Gamma$ (i.e. either $D$ or $f_{r c} / f_{s}$ or both are small), and corresponding small values of $f_{o}$, we have:

$$
G_{S E}\left(f_{o}-n f_{s}\right) \approx \frac{\operatorname{sinc}(D n) e^{-j \pi D n}}{1+j \frac{f_{o}}{D f_{r c}}}, \Gamma \ll 2 .
$$

For the mixing region, first the exact HTFs (3.8) are put into an alternate form (where $f_{i}=f_{o}-n f_{s}$ ):

$$
\begin{aligned}
& H_{n, S E}\left(f_{o}\right)=D \frac{1}{1+j \frac{f_{o}}{D f_{r c}}} \frac{1-e^{-j 2 \pi D n}}{j 2 \pi D n} \\
& \quad+(1-D) \frac{e^{j 2 \pi(1-D) f_{o} / f_{s}}-1}{j 2 \pi(1-D) f_{o} / f_{s}} G_{S E}\left(f_{i}\right)+(1-D) \frac{j \frac{f_{o}}{f_{r c}}}{1+j \frac{f_{o}}{f_{r c}}} \\
& \quad\left[\frac{1}{1+j \frac{f_{o}}{D f_{r c}}} \frac{1-e^{-j 2 \pi D n}}{j 2 \pi D n}-\frac{e^{j 2 \pi(1-D) f_{o} / f_{s}}-1}{j 2 \pi(1-D) f_{o} / f_{s}} G_{S E}\left(f_{i}\right)\right] .
\end{aligned}
$$

For small $f_{o}$ and small $D f_{r c}$ :

$$
\frac{e^{j 2 \pi(1-D) f_{o} / f_{s}}-1}{j 2 \pi(1-D) f_{o} / f_{s}} G_{S E}\left(f_{o}-n f_{s}\right) \approx G_{S E}\left(f_{o}-n f_{s}\right),
$$


so that (A.44) gives:

$$
\frac{e^{j 2 \pi(1-D) f_{o} / f_{s}}-1}{j 2 \pi(1-D) f_{o} / f_{s}} G_{S E}\left(f_{o}-n f_{s}\right) \approx \frac{1}{1+j \frac{f_{o}}{D f_{r c}}} \frac{1-e^{-j 2 \pi D n}}{j 2 \pi D n} .
$$

Therefore the bracketed term in (A.45) vanishes and the HTFs for the mixing region become:

$$
H_{n, S E}\left(f_{o}\right) \approx \frac{\operatorname{sinc}(D n)}{1+j \frac{f_{o}}{D f_{r c}}} e^{-j \pi D n} \quad, \Gamma \ll 2 .
$$

The maximum value of the transfer function depends on the sinc of $D \cdot n$. For each frequency shift, lowpass filtering as a function of output frequency occurs.

\section{A.3.2 Sampling Region}

The sampling region was defined by $\Gamma \gg 2$. The duty cycle is bounded, $0 \leq D \leq 1$, so (3.10) has to be considered for large values of $f_{r c}$ :

$$
G_{S E}\left(f_{i}\right) \approx e^{-j 2 \pi f_{i} / f_{s}(1-D)} \frac{1}{1+j \frac{f_{i}}{f_{r c}}}, \Gamma \gg 2 .
$$

The substitution of (A.49) into (3.8) gives the HTFs for the sampling region:

$$
\begin{aligned}
& H_{n, S E}\left(f_{o}\right) \approx \frac{1}{1+j \frac{f_{o}}{f_{r c}}}[\underbrace{D \operatorname{sinc}(D n) e^{-j \pi D n}}_{\text {Part A }} \\
& +\underbrace{(1-D) \frac{\operatorname{sinc}\left((1-D) f_{o} / f_{s}\right)}{1+j \frac{f_{o}-n f_{s}}{f_{r c}}} e^{-j \pi(1-D) f_{o} / f_{s}} e^{-j 2 \pi D n}}_{\text {Part B }}], \Gamma \gg 2 .
\end{aligned}
$$

From the two terms, part $\mathrm{A}$ is the contribution of the track interval when the switch is closed. Part B is the contribution of the hold interval when the switch is opened.

\section{A.4 Derivation of Kernel Noise}

This section derives the output noise of the SE kernel, using the LPTV noise equation:

$$
N_{o}\left(f_{o}\right)=N_{i} \sum_{n=-\infty}^{\infty}\left|H_{n}\left(f_{o}\right)\right|^{2} .
$$

where $N_{i}=2 k T R$ is the input noise due to the total loop resistance $R$. The PSD will also be integrated over $f_{o}$ to find the total noise power $P_{o}$ :

$$
P_{o}=\int_{-\infty}^{\infty}\left|N_{o}\left(f_{o}\right)\right|^{2} d f_{o}
$$




\section{A.4.1 Mixing Region}

The HTFs for the mixing region have a factor solely depending on $n$ and a low pass factor solely depending on $f_{o}$. Therefore, we first calculate the PSD for zero output frequency and then multiply by the equivalent noise bandwidth to get the total output noise power. Summing (A.48) over $n$ for $f_{o}=0$ according to (A.51) and using (B.5) gives:

$$
\begin{aligned}
N_{o}(0) & =N_{i} \sum_{n=-\infty}^{\infty} \operatorname{sinc}^{2}(D n) \\
& =2 k T R \frac{1}{D} \quad, \Gamma \ll 2 .
\end{aligned}
$$

Using (B.3), the equivalent noise bandwidth $B_{n}$ is:

$$
B_{n}=\int_{-\infty}^{\infty}\left|\frac{1}{1+j \frac{f_{o}}{D f_{r c}}}\right|^{2} d f_{o}=\frac{D}{2 R C}
$$

so that the total noise power is equal to that of an LTI RC network:

$$
P_{o}=N_{o}(0) \cdot B_{n}=\frac{k T}{C}
$$

\section{A.4.2 Sampling Region}

The same strategy can be used for the sampling region HTFs (A.50). For parts A and $\mathrm{B}$ in (A.50):

$$
|A+B|^{2}=|A|^{2}+A B^{*}+A^{*} B+|B|^{2},
$$

where ${ }^{*}$ denotes the complex conjugate. Therefore, the sum over $n$ in (A.51) for zero output frequency $\left(f_{i} / f_{s}=-n\right)$ is expressed as:

$$
\begin{aligned}
& N_{o}(0)=N_{i} \sum_{n=-\infty}^{\infty}[\underbrace{D^{2} \operatorname{sinc}^{2}(D n)}_{A A^{*}}+\underbrace{(1-D)^{2}\left|\frac{1}{1-j \frac{n f_{s}}{f_{r c}}}\right|^{2}}_{B B^{*}} \\
& +\underbrace{(1-D) \frac{D \operatorname{sinc}(D n)}{1+j \frac{n f_{s}}{f_{r c}}} e^{j \pi D n}}_{A B^{*}}+\underbrace{(1-D) \frac{D \operatorname{sinc}(D n)}{1-j \frac{n f_{s}}{f_{r c}}} e^{-j \pi D n}}_{A^{*} B} .
\end{aligned}
$$

Using (B.4)-(B.6), this evaluates to:

$$
N_{o}(0) \approx 2 k T[\underbrace{D R}_{A A^{*}}+\underbrace{\frac{(1-D)^{2}}{2 f_{s} C}}_{B B^{*}}+\underbrace{2(1-D) R}_{A B^{*}+A^{*} B}], \Gamma \gg 2 .
$$


Note that for $\Gamma \gg 2$ the $B B^{*}$ term, contributed by the hold interval, is dominant.

Finding the equivalent noise bandwidths of these terms is complicated because of the low pass input filter in (A.50). The calculations are made easier by assuming that:

- Part A is dominated in bandwidth by $\frac{1}{1+j \frac{f_{o}}{f_{r c}}}$,

- Part B is dominated in bandwidth by $\operatorname{sinc}\left((1-D) f_{o} / f_{s}\right)$,

- The integrated cross terms $A B^{*}$ and $A^{*} B$ are zero.

Part A and part B do not overlap in the time domain, therefore the integrated crosspower spectrum must be zero according to Parseval's theorem:

$$
\int_{-\infty}^{\infty} A(f) B^{*}(f) d f=\int_{-\infty}^{\infty} a(t) b^{*}(t) d t=0 .
$$

Using (B.2) and (B.3), the noise equivalent bandwidths are:

$$
B_{n, A A^{*}}=\int_{-\infty}^{\infty}\left|\frac{1}{1+j \frac{f_{o}}{f_{r c}}}\right|^{2} d f_{o}=\frac{1}{2 R C},
$$

and

$$
B_{n, B B^{*}}=\int_{-\infty}^{\infty} \operatorname{sinc}^{2}\left((1-D) \frac{f_{o}}{f_{s}}\right) d f_{o}=\frac{f_{s}}{1-D} .
$$

So we see that the track interval (Part A) has a lower low-frequency PSD, but a much higher noise bandwidth than the hold interval (Part B). If we calculate the total noise power:

$$
\begin{aligned}
P_{o} & =N_{o, A A^{*}}(0) \cdot B_{n, A A^{*}}+N_{o, B B^{*}}(0) \cdot B_{n, B B^{*}} \\
& =D \frac{k T}{C}+(1-D) \frac{k T}{C}=\frac{k T}{C},
\end{aligned}
$$

we find that the total integrated noise is the well known $k T / C$ for sampled-data systems. It is distributed proportionally to interval length over the track and hold interval. 


\section{Appendix B}

\section{Integrals and Sums}

In this appendix the parameters $a$ and $b$ are real numbers. The sinc function is defined as:

$$
\begin{aligned}
& \operatorname{sinc}(x) \equiv \frac{\sin \pi x}{\pi x}=\frac{e^{j \pi x}-e^{-j \pi x}}{j 2 \pi x} \\
& \operatorname{sinc}(0)=1, \quad \Im(\operatorname{sinc}(x))=0
\end{aligned}
$$

Integrals using ([72] 3.821 9.) and ([72] 3.1122.$)$ :

$$
\begin{gathered}
\int_{-\infty}^{\infty}\left|\frac{1-e^{-j 2 \pi a x}}{j 2 \pi x}\right|^{2} d x=\frac{2}{\pi^{2}} \int_{0}^{\infty} \frac{\sin ^{2}(\pi a x)}{x^{2}} d x=a \\
\int_{-\infty}^{\infty}\left|\frac{1}{1+j b x}\right|^{2} d x=\int_{-\infty}^{\infty} \frac{1}{(1+j b x)(1-j b x)} d x=\frac{\pi}{b}
\end{gathered}
$$

Sums using ([72] $\mathbf{1 . 2 1 7} 1$.$) , ([72] \mathbf{0 . 2 3 3})$ and ([72] 1.4433.$)$ :

$$
\begin{aligned}
& \sum_{n=-\infty}^{\infty}\left|\frac{1}{1-j b n}\right|^{2}=1+2 \sum_{n=1}^{\infty} \frac{1}{1+b^{2} n^{2}}=\frac{\frac{\pi}{b}}{\tanh \left(\frac{\pi}{b}\right)} \\
& \approx \frac{\pi}{b}, \quad 0<b \ll 1 \\
& \sum_{n=-\infty}^{\infty}\left|\frac{1-e^{-j 2 \pi a n}}{j 2 \pi n}\right|^{2}=a^{2}+2 \sum_{n=1}^{\infty} \frac{\sin ^{2} \pi a n}{\pi^{2} n^{2}} \\
&=a^{2}+\frac{1}{\pi^{2}}\left[\sum_{n=1}^{\infty} \frac{1}{n^{2}}-\sum_{n=1}^{\infty} \frac{\cos 2 \pi a n}{n^{2}}\right]=a
\end{aligned}
$$


Sum using ([72] 1.441 1.), ([72] 1.217 1.), ([72] 1.4452 .) and ([72] 1.4451.$)$ :

$$
\begin{array}{r}
\sum_{n=-\infty}^{\infty} \frac{e^{j 2 \pi a n}-1}{j 2 \pi n(1+j b n)}=\sum_{n=-\infty}^{\infty} \frac{\left(e^{j 2 \pi a n}-1\right)(1+j b n-j b n)}{j 2 \pi n(1+j b n)} \\
=\sum_{n=-\infty}^{\infty} \frac{e^{j 2 \pi a n}-1}{j 2 \pi n}-b \sum_{n=-\infty}^{\infty} \frac{e^{j 2 \pi a n}-1}{2 \pi(1+j b n)} \\
=a+2 \sum_{n=1}^{\infty} \frac{\sin (2 \pi a n)}{2 \pi n}+\sum_{n=-\infty}^{\infty} \frac{b}{2 \pi\left(1+b^{2} n^{2}\right)} \\
-\sum_{n=-\infty}^{\infty} \frac{b \cos (2 \pi a n)}{2 \pi\left(1+b^{2} n^{2}\right)}-\sum_{n=-\infty}^{\infty} \frac{b^{2} n \sin (2 \pi a n)}{2 \pi\left(1+b^{2} n^{2}\right)} \\
=\frac{1}{2}+\frac{1}{2} \cdot \frac{1+e^{-2 \pi b^{-1}}}{1-e^{-2 \pi b^{-1}}}-\frac{e^{-2 \pi a b^{-1}}}{1-e^{-2 \pi b^{-1}}}, \quad 0<a<1 \\
\approx 1, \quad b \rightarrow 0
\end{array}
$$




\section{Dankwoord}

Het uitvoeren en voltooien van een promotie traject, resulterend in een proefschrift, wordt vaak gezien als een individuele prestatie van de promovendus. In werkelijkheid is het echter een teamprestatie, waarvan het wel slagen valt of staat met de inbreng en invloed van de mensen om de promovendus heen. Dit dankwoord is gericht aan jullie.

Beginnend bij mijn promotor Frank, mijn rots in de branding gedurende het schrijven van dit proefschrift. Jouw overzicht over het grote geheel en aandacht voor de wetenschappelijke inhoud waren van onschatbare waarde om mijn enthousiaste claims te temperen en om te vormen naar solide wetenschappelijke bijdragen. Speciale dank voor mijn dagelijkse begeleider en assistent promotor Eric, voor het doorstaan van alle energierijke discussies tijdens de projectvergaderingen en het in banen leiden van het onderzoek. Er is geen mens op deze aarde geduldiger en aandachtiger dan jij. Voor creatieve inbreng en brainstorming richtte ik mij tot professor Bram N. (a.k.a. grote Bram). Het is af en toe eng hoe strak wij staan afgesteld op dezelfde golflengte, waarbij enkele woorden of lijnen op het whiteboard genoeg zijn om de essentie van een ontwerp over te brengen. Acht jaar geleden was jij degene die mij na een college Electronic Signal Processing vertelde over geïntegreerde analoge elektronica, dat moment was voor mij de aanzet om chip-ontwerper te geworden. Het was misschien geen toeval dat dat gesprek ook al over mixers ging..

Natuurlijk is ondersteuning vanuit de industrie cruciaal voor het afronden van de chip ontwerpen. Speciale dank voor Domine van NXP voor alle ondersteuning gedurende de eerste helft van het project, met inbegrip van mijn allereerste ontwerp review, waar ik alle basis technieken van chip layout heb opgedaan. Many thanks as well to Andreia of ST Microelectronics, who supported me during the second half of the project and even offered the hospitality of her home when all hotels were booked in Grenoble: merci beaucoup.

Zoals alle promovendi weten, zijn het de details die een ontwerp kunnen maken of breken. Wat ontbreekt in de uiteindelijke presentatie zijn de bloed, zweet en tranen die vergoten zijn tijdens de late uurtjes van het layout design en de metingen. Daarom wil ik de aandacht vestigen op het fantastische werk dat gedaan wordt door Henk en 
Gerard. Zij zijn degenen die te maken krijgen met alle overblijvende problemen en zonder hun hulp zouden er geen chips en metingen zijn om over te publiceren.

Naast het serieuze werk is een gezonde dosis humor, afleiding en absurditeit noodzakelijk om gezond van geest te blijven. Gelukkig waren deze kwaliteiten in overmate aanwezig bij mijn kamergenoten bij ICD. Jasper, Johan, Erik, Bram V. (a.k.a. kleine Bram), Harish, David en Mark: bedankt voor alle zinvolle en zinloze discussies, nachtelijke uitstapjes en maffe projectjes (vaak eindigend met de kalibratiehamer). Verder wil ik Gerdien en Annemiek bedanken voor het draaiend houden van de vakgroepen en het bakje goeie koffie dat ik af en toe mocht bietsen. Natuurlijk ook vele dank aan mijn andere collega's bij ICD, SC en TE voor de prettige werkomgeving en leuke afleidingen zoals diners en barbecues.

Buiten werk om is het ook belangrijk om af en toe stoom af te blazen. Sander, Niels en Hidde, studiegenoten vanaf het eerste uur, wat hebben we veel lol gehad in al die jaren. Hoewel we verschillende wegen hebben bewandeld zijn we dan toch aangekomen bij het eindpunt van onze studies en zullen we nu toch echt moeten integreren in werkend Nederland. Bedankt voor jullie vriendschap door weer en wind.

Dan nu speciale aandacht voor mijn paranimfen Peter en Gerjan. Naast jullie ondersteuning tijdens de weekeinden op rij 13, vak 306 in de Grolsch Veste ben ik vereerd jullie naast mij te hebben staan tijdens de verdediging van dit proefschrift. We kunnen nu toch duidelijk stellen dat we samen met Berrie en Jouke de juiste beslissing hebben genomen al die jaren terug om door te gaan met de MEK, met als gevolg vriendschappen voor het leven. Natuurlijk is ons groepje niet compleet zonder Erwin, hoe zouden we anders een biertje van het huis krijgen bij eetcafé Jeruzalem? Dat we samen nog vele shoarma's mogen proeven.

Ik wil ook de voormalige en huidige bewoners van de MEK bedanken voor alle gezelligheid en voor het feit dat oud-MEKkers nog steeds hartelijk welkom worden geheten op de vled (nee, dit is geen typo). De vraag komt wel naar boven of jullie voortaan zelf de telefoonrekening gaan betalen (knipoog).

Als laatste wil ik mijn hele familie veel dank en liefde toezenden, voor alle ondersteuning gedurende mijn studie en promotie. Met name mijn ouders voor de stabiele achtergrond die zij mij hebben gegeven. Marloes, Jarno en Karina, ik bedankt jullie natuurlijk ook! Ik wil afsluiten door even stil te staan bij de vooruitziende blik van Opa en Oma Evers. Zij gaven mij als klein jongetje een elektronica experimenteerdoos op mijn communiefeest. $\mathrm{U}$ ziet voor $\mathrm{u}$ het resultaat liggen. 


\section{Bibliography}

[1] J. Kilby, "Miniature Electronic Circuits," US Patent 3138 743, 1963.

[2] S. Damaraju, V. George, S. Jahagirdar, T. Khondker, R. Milstrey, S. Sarkar, S. Siers, I. Stolero, and A. Subbiah, "A 22nm IA Multi-CPU and GPU Systemon-Chip," IEEE ISSCC Dig. Tech. Papers, vol. 55, pp. 56-57, 2012.

[3] International Technology Roadmap for Semiconductors. [Online]. Available: http://www.itrs.net

[4] R. H. Dennard, F. H. Gaensslen, H. Yu, R. V. Leo, E. Bassous, and A. R. LeBlanc, "Design of Ion-Implanted MOSFETs with Very Small Physical Dimensions," IEEE Journal of Solid-State Circuits, vol. 9, pp. 256-268, Oct. 1974.

[5] L. Zheng and D. N. C. Tse, "Diversity and Multiplexing: A Fundamental Tradeoff in Multiple-Antenna Channels," IEEE Trans. on Information Theory, vol. 49, no. 5, pp. 1073-1096, May. 2003.

[6] T. Paul and T. Ogunfunmi, "Wireless LAN Comes of Age: Understanding the IEEE 802.11n Amendment," IEEE Circuits and Systems Magazine, pp. 28-54, 2008.

[7] A. Ghosh, R. Ratasuk, B. Mondal, N. Mangalvedhe, and T. Thomas, "LTEAdvanced: Next-Generation Wireless Broadband Technology," IEEE Wireless Communications, pp. 10-22, Jun. 2010.

[8] J. Paramesh, R. Bishop, K. Soumyanath, and D. J. Allstot, "A 1.4V 5GHz FourAntenna Cartesian-Combining Receiver in 90nm CMOS for Beamforming and Spatial Diversity Applications," IEEE ISSCC Dig. Tech. Papers, pp. 210-211, 2005 .

[9] K. G. Jansky, "Directional Studies of Atmospherics at High Frequencies," Proc. of the Institute of Radio Engineers, vol. 20, no. 12, pp. 1920-1932, Dec. 1932. 
[10] H. T. Friss and C. B. Feldman, "A Multiple Unit Steerable Antenna for ShortWave Reception," Proc. of the Institute of Radio Engineers, vol. 25, no. 7, pp. 841-917, Jul. 1937.

[11] M. I. Skolnik, Introduction to Radar Systems. McGraw Hill, 1981.

[12] J. Frank, C. A. Shipley, and J. H. Kuck, "Latching ferrite phase shifter for phased arrays," Microwave Journal, pp. 97-102, 1967.

[13] W. J. Ince, "Recent advances in diode and phase shifter technology for phased array radars," Microwave Journal, vol. 15, no. 9, pp. 36-46, 1972.

[14] J. Sneider, "The design construction and test results of the CAISSA planar space fed phased array antenna," On Microwave Measurement, Transmission and Reception, 1978.

[15] D. McQuiddy, R. Gassner, P. Hull, J. Mason, and J. Bedinger, "Transmit/Receive Module Technology for X-Band Active Array Radar," Proc. IEEE, vol. 79, pp. 308-341, Mar. 1991.

[16] J. Bugeau, M. P. W. Coughlin, and G. Onge, "Advanced MMIC T/R Module for 6 to $18 \mathrm{GHz}$ Multifunction Arrays," IEEE Microwave Symposium Digest, pp. 81-84, 1992.

[17] M. van Heijningen, A. de Boer, J. A. Hoogland, M. van Wanum, A. P. de Hek, F. E. van Vliet, and H. Brouzes, "Multi Function and High Power Amplifier Chipset for X-Band Phased Array Frontends," European Microwave Integrated Circuits Conf., pp. 237-240, 2006.

[18] F. E. van Vliet and A. de Boer, "Fully-Integrated Core chip for X-Band PhasedArray T/R modules," IEEE Microwave Symposium Digest, vol. 3, pp. 1753-1756, 2004.

[19] K. Koh and G. M. Rebeiz, "An X- and Ku-band 8-Element Phased-Array Receiver in 0.18-um SiGe BiCMOS Technology," IEEE Journal of Solid-State Circuits, vol. 43, no. 6, pp. 1360-1371, Jun. 2008.

[20] H. Hashemi, X. Guan, A. Komijani, and A. Hajimiri, "A 24-Ghz SiGe PhasedArray Receiver - LO Phase Shifting Approach," IEEE Trans. on Microwave Theory and Techniques, vol. 53, no. 2, pp. 614-626, Feb. 2005.

[21] A. Babakhani, X. Guan, A. Komijani, and A. Hajimiri, "A 77 GHz 4-Element Phased Array Receiver with On-Chip Dipole Antennas in Silicon," IEEE ISSCC Dig. Tech. Papers, pp. 629-638, 2006. 
[22] S. Y. Kim and G. M. Rebeiz, "A Low Power BiCMOS 4-Element Phased Array Receiver for 76-84 GHz Radars and Communication Systems," IEEE Journal of Solid-State Circuits, vol. 47, no. 2, pp. 359-367, Feb. 2012.

[23] T. Yu and G. M. Rebeiz, "A 22-24 GHz 4-Element CMOS Phased Array With On-Chip Coupling Characterization," IEEE Journal of Solid-State Circuits, vol. 43, no. 9, pp. 2134-2143, Sep. 2008.

[24] E. Cohen, C. Jakobson, S. Ravid, and D. Ritter, "A thirty two element phasedarray transceiver at $60 \mathrm{GHz}$ with RF-IF conversion block in 90nm flip chip CMOS process," IEEE Radio Frequency Integrated Circuits Symposium, pp. 457-460, 2010.

[25] K. Raczkowski, W. D. Raedt, B. Nauwelaers, and P. Wambacq, "A Wideband Beamformer for a Phased-Array 60GHz Receiver in 40nm Digital CMOS," IEEE ISSCC Dig. Tech. Papers, pp. 40-41, 2010.

[26] S. Emami, R. F. Wiser, E. Ali, M. G. Forbes, M. Q. Gordon, X. Guan, S. Lo, P. T. McElwee, J. Parker, J. R. Tani, J. M. Gilbert, and C. H. Doan, "A 60 GHz CMOS Phased-Array Transceiver Pair for Multi-Gb/s Wireless Communications," IEEE ISSCC Dig. Tech. Papers, pp. 164-165, 2011.

[27] A. Annema, B. Nauta, R. van Langevelde, and H. Tuinhout, "Analog Circuits in Ultra-Deep-Submicron CMOS," IEEE Journal of Solid-State Circuits, vol. 40, no. 1, pp. 132-143, Jan. 2005.

[28] R. C. Hansen, Microwave Scanning Antenna, Volume II Array Theory and Practice. New York: Academic, 1966.

[29] C. A. Balanis, Antenna Theory, Analysis and Design. John Wiley \& Sons, inc, New York, 2005.

[30] R. J. Mailloux, Phased Array Antenna Handbook. Artech House, 2005.

[31] R. S. Elliot, "Beamwidth and Directivity of large Scanning Arrays," Microwave Journal, pp. 74-82, 1964.

[32] M. Skolnik, "Resolution of Angular Ambiguities in Radar Array Antennas with Widely-Spaced Elements and Grating Lobes," IRE Trans. on Antennas and Propagation, pp. 351-352, 1962.

[33] J. Frank, "Bandwidth criteria for phased array antennas," Phased Array Antennas, pp. 243-253, 1972. 
[34] C. L. Dolph, "A Current Distribution for Broadside Arrays Which Optimizes the Relationship Between Beamwidth and Side-Lobe Level," Proc. IRE and Waves and Electrons, Jun. 1946.

[35] T. T. Taylor, "Design of Line Source Antennas for Narrow Beamwidth and Low Sidelobes," IEEE Trans. on Antennas and Propagation, pp. 16-28, 1955.

[36] S. P. Applebaum, "Adaptive Arrays," IEEE Trans. on Antennas and Propagation, pp. 585-598, 1976.

[37] M. Skolnik, Radar Handbook. McGraw Hill, 2008.

[38] J. L. Allen, The theory of array antennas. MIT Lincoln Lab. Report 323, 1963.

[39] C. J. Miller, "Minimizing the Effects of Phase Quantization Errors in an Electronically Scanned Array," Proc. 1964 Symp. Electronically Scanned Phased Arrays and Applications, vol. 1, pp. 17-39, 1964.

[40] R. J. Mailloux, "Array Grating Lobes Due to Periodic Phase, Amplitude and Time Delay Quantization," IEEE Trans. on Antennas and Propagation, pp. 1364-1368, 1984.

[41] B. Razavi, RF Microelectronics. Prentice Hall New Jersey, 2006.

[42] A. R. Shahani, D. K. Shaeffer, and T. H. Lee, "A 12-mW Wide Dynamic Range CMOS Front-End for a Portable GPS Receiver," IEEE Journal of Solid-State Circuits, vol. 32, no. 12, Dec 1997.

[43] B. Leung, VLSI for Wireless Communication. Prentice Hall New Jersey, 2002.

[44] A. Parssinen, R. Magoon, S. I. Long, and V. Porra, "A 2-GHz Subharmonic Sampler for Signal Downconversion," IEEE Trans. on Microwave Theory and Techniques, vol. 45, no. 12, Dec 1997.

[45] R. Gregorian and G. C. Temes, Analog MOS Integrated Circuits for Signal Processing. John Wiley \& Sons, inc, 1986.

[46] D. R. Tayloe, "Product detector and method therefor," US Patent 6230000 , 2001.

[47] B. W. Cook, A. Berny, A. Molnar, S. Lanzisera, and K. S. J. Pister, "Low-Power 2.4-GHz Transceiver With Passive RX Front-End and 400-mV Supply," IEEE Journal of Solid-State Circuits, vol. 41, no. 12, Dec 2006.

[48] P. Vanassche, G. Gielen, and W. Sansen, "Symbolic Modelling of Periodically Time-Varying Systems Using Harmonic Transfer Matrices," IEEE Trans. on CAD of Integrated Circuits and Systems, vol. 21, no. 9, Sep 2002. 
[49] C. L. Phillips and J. M. Parr, Signals, Systems and Transforms. Prentice-Hall, 1995.

[50] H. H. Rosenbrock, State-Space and Multivariable Theory. Nelsen, 1970.

[51] T. Ström and S. Signell, "Analysis of Periodically Switched Linear Circuits," IEEE Trans. on Circuits and Systems, vol. CAS-24, no. 10, Oct 1977.

[52] M. L. Liou and Y. Kuo, "Exact Analysis of Switched Capacitor Circuits with Arbitrary Inputs," IEEE Trans. Circuits and Systems, vol. CAS-26, no. 4, Apr 1979.

[53] K. Kundert. Simulating Switched-Capacitor Filters with SpectreRF. [Online]. Available: http://www.designers-guide.org/analysis/sc-filters.pdf

[54] M. Camus, "A 5.4mw 0.07mm2 2.4ghz front-end receiver in 90nm cmos for ieee 802.15.4 wpan," IEEE ISSCC Dig. Tech. Papers, pp. 368-620, Feb. 2008.

[55] S. Patnaik, N. Lanka, and R. Harjani, "A Dual-Mode Architecture for a Phased-

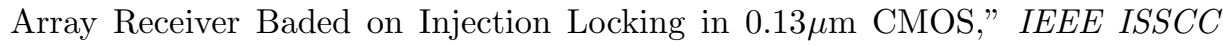
Dig. Tech. Papers, pp. 490-491, 2009.

[56] K. Scheir, S. Bronckers, J. Borremans, P. Wambacq, and Y. Rolain, "A 52GHz Phased-Array Receiver Front-End in 90nm Digital CMOS," IEEE ISSCC Dig. Tech. Papers, pp. 184-185, Feb. 2008.

[57] S. Jeon, Y. Wang, H. Wang, F. Bohn, A. Natarajan, A. Babakhani, and A. Hajimiri, "A Scalable 6-to-18GHz Concurrent Dual-Band Quad-Beam PhasedArray Receiver in CMOS," IEEE Journal of Solid-State Circuits, vol. 43, pp. 2660-2673, 2008.

[58] C. Andrews and A. C. Molnar, "A Passive-Mixer-First Receiver with BasebandControlled RF Impedance Matching, < 6dB NF, and >27dBm Wideband IIP3," IEEE ISSCC Dig. Tech. Papers, vol. 53, pp. 46-47, 2010.

[59] L. E. Franks and I. W. Sandberg, "An alternative approach to the realization of network transfer functions: The N-path Filters," Bell Sys. Tech. J., vol. 39, pp. 1321-1350, 1960.

[60] R. E. Collin, Foundations for Microwave Engineering. McGraw-Hill, N.Y., 1992.

[61] D. M. Pozar, Microwave Engineering. Wiley and Sons, Inc., 2005.

[62] C. Andrews and A. C. Molnar, "Implications of Passive Mixer Transparency for Impedance Matching and Noise Figure in Passive Mixer-First Receivers," IEEE Trans. on Circuits and Systems I, vol. 57, pp. 3092-3103, 2010. 
[63] A. Mirzaei, H. Darabi, and D. Murphy, "Architectural Evolution of Integrated M-Phase High-Q Bandpass Filters," IEEE Trans. Circuits and Systems-I, vol. 59, no. 1, pp. 52-65, Jan. 2012.

[64] J. Borremans, G. Mandal, V. Giannini, T. Sano, M. Ingels, B. Verbruggen, and J. Craninckx, "A 40nm CMOS Highly Linear 0.4-to6Ghz Receiver Resilient to 0dBm Out-of-Band Blockers," IEEE ISSCC Dig. Tech. Papers, pp. 62-63, 2011.

[65] D. Murphy, A. Hafez, A. Mirzaei, M. Mikhemar, H. Darabi, M. F. Chang, and A. Abidi, "A Blocker-Tolerant Wideband Noise-Cancelling Receiver With a $2 \mathrm{~dB}$ Noise Figure," IEEE ISSCC Dig. Tech. Papers, pp. 74-75, 2012.

[66] A. Ghaffari, E. A. M. Klumperink, and B. Nauta, "A Differential 4-Path Highly Linear Widely Tunable On-Chip Band-Pass Filter," IEEE Radio Frequency Integrated Circuits Symposium, pp. 299-302, 2010.

[67] A. Mirzaei, H. Darabi, A. Yazdi, Z. Zhou, E. Chang, and P. Suri, "A 65nm CMOS Quad-Band SAW-Less Receiver SoC for GSM/GPRS/EDGE," IEEE Journal of Solid-State Circuits, vol. 46, no. 4, pp. 950-964, Apr. 2011.

[68] M. Darvishi, R. van der Zee, E. A. M. Klumperink, and B. Nauta, "A 0.3to-1.2GHz Tunable 4-th Order Switched Gm-C Bandpass Filter with > 55dB Ultimate Rejection and Out-of-Band IIP3 of +29dBm," IEEE ISSCC Dig. Tech. Papers, pp. 358-359, 2012.

[69] R. Tseng, H. Li, D. H. Kwon, Y. Chiu, and A. S. Y. Poon, "A Four-Channel Beamforming Down-Converter in 90-nm CMOS Utilizing Phase-Oversampling," IEEE Journal of Solid-State Circuits, vol. 45, no. 11, pp. 2262-2272, Nov. 2010.

[70] B. Cetinoneri, Y. A. Atesal, and G. M. Rebeiz, "An 8 X 8 Butler Matrix in 0.13um CMOS for 5-6 GHz Multibeam Applications," IEEE Trans. on Microwave Theory and Techniques, vol. 59, no. 2, pp. 295-301, Feb. 2011.

[71] A. Opal and J. Vlach, "Analysis and Sensitivity of Periodically Switched Linear Networks," IEEE Trans. on Circuits and Systems, vol. 36, no. 4, Apr 1989.

[72] I. S. Gradshteyn and I. M. Ryzhik, Table of Integrals, Series, and Products, A. Jeffrey, Ed. Academic Press, 1994. 


\section{List of Publications}

1. M.C.M. Soer, E.A.M. Klumperink, Z. Ru, F.E. van Vliet, B. Nauta, "A 0.2to-2.0GHz 65nm CMOS Receiver Without LNA Achieving > $>11 \mathrm{dBm}$ IIP3 and $<6.5 \mathrm{~dB}$ NF", IEEE Solid-State Circuits Conference, Digest of Technical Papers, pp. 222-223, Feb 2009.

2. M.C.M. Soer, E.A.M. Klumperink, PT de Boer, F.E. van Vliet, B. Nauta, "Unified Frequency Domain Analysis of Switched-RC Passive Mixers and Samplers", IEEE Transactions on Circuits and Systems I: Regular Papers, vol. 57, num. 10, pp. 2618-2631, Oct. 2010.

3. M.S. Oude Alink, E.A.M. Klumperink, M.C.M. Soer, A.B.J. Kokkeler, B. Nauta, "A 50MHz-to-1.5GHz Cross-Correlation CMOS Spectrum Analyzer for Cognitive Radio with $89 \mathrm{~dB}$ SFDR in $1 \mathrm{MHz}$ RBW", Proc. 4th IEEE Symposium on New Frontiers in Dynamic Spectrum Access Networks, Singapore, Apr. 2010.

4. M.C.M. Soer, E.A.M. Klumperink, B. Nauta, F.E. van Vliet, "A 1.0-to4.0GHz 65nm CMOS Four-Element Beamforming Receiver Using a SwitchedCapacitor Vector Modulator with Approximate Sine Weighting via Charge Redistribution", IEEE Solid-State Circuits Conference, Digest of Technical Papers, pp. 64-65, Feb 2011.

5. A. Ghaffari, E.A.M. Klumperink, M.C.M. Soer, B. Nauta, "Tunable High-Q N-Path Band-Pass Filters: Modeling and Verification", IEEE Journal of SolidState Circuits, vol. 46, num. 5, pp. 998, May 2011.

6. M.C.M. Soer, E.A.M. Klumperink, B. Nauta, F.E. van Vliet, "Spatial Interferer Rejection in a 4-Element Beamforming Receiver Frontend with a SwitchedCapacitor Vector Modulator", IEEE Journal of Solid-State Circuits, vol.46, no.12, pp. 2933-2942, Dec. 2011. 
7. M.S. Oude Alink, E.A.M. Klumperink, A.B.J. Kokkeler, M.C.M. Soer, G.J.M. Smit, B. Nauta, " A CMOS-Compatible Spectrum Analyzer for Cognitive Radio Exploiting Crosscorrelation to Improve Noise and Linearity Performance", IEEE Transactions on Circuits and Systems I: Regular Papers, vol. 59, num. 3, pp. 479-492, Mar. 2012.

8. M.C.M. Soer, E.A.M. Klumperink, B. Nauta, F.E. van Vliet, "A 1.5-to5.0GHz Input-Matched +2dBm P1dB All-Passive Switched-Capacitor Beamforming Receiver Front-End in 65nm CMOS", IEEE Solid-State Circuits Conference, Digest of Technical Papers, pp. 174-175, Feb 2012.

9. F.E. van Vliet, E.A.M. Klumperink, M.C.M. Soer, S.K. Garakoui, A. de Boer, A.P. de Hek, W. de Heij, B. Nauta, "Advances in Silicon Phased-Array Receiver IC's", IEEE International Microwave Symposium, Digest of Technical Papers, Jun. 2012. 


\section{List of Abbreviations}

$\mathrm{AC}$

$\mathrm{ADC}$

CML

CMOS

DC

DI

EM

FET

GaAs

GaN

HTF

IC

IEEE

IF

IIP3

$\mathrm{InP}$

$\mathrm{I} / \mathrm{Q}$

ISM

LNA

LO

LP

LPTV

LTE
Alternating Current

Analog to Digital Converter

Current Mode Logic

Complementary Metal Oxide Semiconductor

Direct Current

Differential

Electro-Magnetic

Field Effect Transistor

Gallium-Arsenide

Gallium-Nitride

Harmonic Transfer Function

Integrated Circuit

Institute of Electrical and Electronics Engineers

Intermediate Frequency

Input-referred third-order Intercept Point

Indium-Phosphide

In-phase / Quadrature

Industrial Scientific Medical

Low Noise Amplifier

Local Oscillator

Low Power

Linear Periodically Time Variant

Long Term Evolution 
LTI Linear Time Invariant

MIMO Multiple-Input-Multiple-Output

MMIC Monolithic Microwave Integrated Circuits

NF Noise Figure

NMOS N-type Metal Oxide Semiconductor

PCB Printed Circuit Board

PMOS P-type Metal Oxide Semiconductor

PSD Power Spectral Density

RC Resistor-Capacitor

RF Radio Frequency

RMS Root Mean Square

SE Single Ended

SFDR Spurious Free Dynamic Range

SiGe Silicon-Germanium

SNR Signal-to-Noise Ratio

SSB Single Side Band

T/R Transmit / Receive

WLAN Wireless Local Area Network

ZOH Zero-Order Hold 